UNITED STATES

DEPARTMENT OF THE INTERIOR

Geological Survey

HYDROLOGIC DATA OF THE COASTAL DRAINAGE BASINS OF SOUTHEASTERN MASSACHUSETTS, NARRAGANSETT BAY, AND RHODE ISLAND SOUND

By

Richard E. Willey, Johṇ R. Williams, and Gary D. Tasker

Massachusefts Hydrologic-Data Report No. 25

Records of selected wells, test wells, borings, municipal water systems, streamflow measurements, and chemical analyses of water in the basins draining into Narragansett Bay and Rhode Island Sound

Prepared in cooperation with the

COMMONWEALTH OF MASSACHUSETTS, WATER RESOURCES COMMISSION

Boston, Massachusetts

OPEN-FILE REPORT 83-145 


\section{CONTENTS}

Introduction

Numbering and location of surface-water stations

Quality of water measurements

Definition of terms

Temperature

Temperature conversion table

Page

Conversion factors -
Selected references

Selected references -

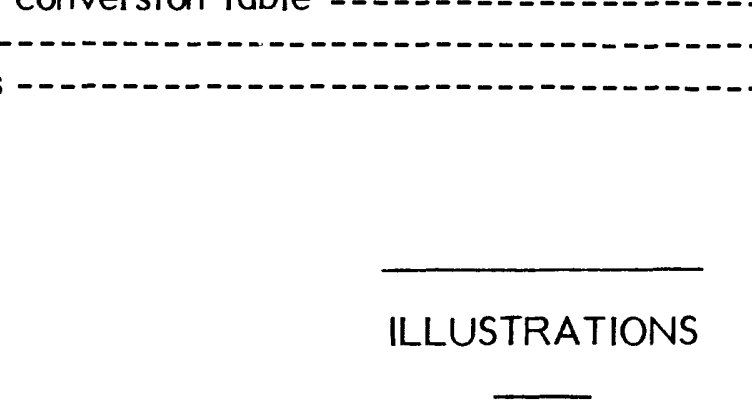

\section{ILLUSTRATIONS}

(Plate is in pocket.)

Plate 1. Map of coastal drainage basins of southeastern Massachusetts, Narragansett Bay, and Rhode Island Sound showing hydrologic-data sites.

\section{TABLES}

Table 1. Description of selected wells, test wells, and borings

2. Logs of selected wells, test wells, and borings

3. Chemical analyses of ground water

5. Discharge, specific conductance, and water temperature measurements at partial-record stations during water years 1972-74

6. Chemical analyses at gaging stations and partial-record stations

8. List of hydrologic-data reports for Massachusetts, New Hampshire, and Maine-- 


\title{
HYDROLOGIC DATA OF THE COASTAL DRAINAGE BASINS OF SOUTHEASTERN MASSACHUSETTS, NARRAGANSETT BAY AND RHODE ISLAND SOUND
}

\author{
By Richard E. Willey, John R. Williams, and Gary D. Tasker
}

\section{INTRODUCTION}

The principal drainage basins are those of the East and West Branches of the Westport River which empty into Rhode Island Sound and of the Lee, Cole, Kickamuit, Palmer, and Runnins Rivers draining to Narragansett Bay. The area includes a small part of the Tenmile River basin that was not included in previous reports (Williams, 1968; Williams and Willey, 1967). The basins are bounded on the east by the Paskamansett-Slocums River basin, on the north by the Taunton' River basin, and on the west by the State of Rhode Island. The study area includes much of the city of Fall River and all or major parts of the towns of Westport, Dartmouth, Swansea, Rehoboth, Seekonk, and small parts of the city of Attleboro and the towns of Somerset, Dighton, and Freetown. Within the basins are the reservoirs (North and South Watuppa Ponds, Noquochoke Lake, and Copicut Reservoir) that supply Fall River, one of two wells supplying the Dighton Water District, and one of three wells supplying Dartmouth. The Seekonk Water District and the Swansea Water District are supplied by water from wells within each town. Attleboro and Somerset are supplied by municipal systems drawing water from reservoirs outside the basin. Rehoboth, Westport, and Freetown have no municipal water systems. The entire area is in Bristol County, Massachusetts.

This report presents, in tabular form, selected records of wells, test wells; borings; measurements of stream discharge, specific conductance, and temperature at the East Branch Palmer River gaging station and at partial-record stations; chemical analyses of ground water and surface water; and a summary of municipal water sources and additional available sources. The data were collected during a study of the drainage basins from 1972 to 1974 in cooperation with the Massachusetts Water Resources Commission. The report is released in order to make available to the public and to local, State, and Federal agencies, basic hydrologic information that may aid in planning water-resources development. Basic records contained in this report and the streamflow data published elsewhere (U.S. Geological Survey, 1954, 1964, 1967, 1968, 1969a, 1969b, 1970, 1971, 1973, 1974, 1975a, 1975b, 1975c, 1976, 1977, 1978, 1979, 1980, 1981) complement an interpretive report (Willey, Williams, and Tasker, 1978).

The authors wish to acknowledge the public officials, consulting firms, industrial concerns, well drillers, and individual homeowners who have given their time and information to this study.

\section{NUMBERING AND LOCATION OF SURFACE-WATER STATIONS}

Records are listed in the order that the main stream enters the ocean, from north to south. Stations on tributaries are listed in the order in which the tributaries enter the main stream in its course to the sea. As an added means of identification, each gaging station and partial-record station has been assigned a number for the U. S. Geological Survey national surface-water data network.

\section{QUALITY OF WATER MEASUREMENTS}

Complete and partial chemical analyses in tables 3,5, and 6 refer to the appropriate well or spring number, to the appropriate gaging-station number or partial-record station number, or to the pond or reservoir name as shown on plate 1. 


\section{DEFINITION OF TERMS}

Definition of terms related to streamflow, water quality, ground water, and other hydrologic data, as used in this report, are defined as follows:

Color is expressed in units of the platinum-cobalt scale proposed by Hazen (1892, p. 427-428). A unit of color is produced by I milligram per liter of platinum in the form of the chloroplatinate ion.

The extent to which water is colored by material in solution is reported as part of the water analysis because a significant color in water may indicate the presence of organic material that may have some bearing on the dissolved-solids content.

Cubic foot per second $\left(\mathrm{ft}^{3} / \mathrm{s}\right)$ is the rate of discharge representing a volume of 1 cubic foot passing a given point during $I$ second and is equivalent to 7.48 gallons per second or 448.8 gallons per minute or 646,317 gallons per day.

Discharge is the volume of water (or more broadly, volume of fluid plus suspended sediment) that passes a given point within a given period of time.

Instantaneous discharge or streamflow is the discharge at a particular instant of time.

Drainage area of a stream at a specific location is that area, measured in a horizontal plane, enclosed by a topographic divide from which direct surface runoff from precipitation normally drains by gravity into the stream above the specified point. Figures of drainage area given herein include all closed basins, or noncontributing areas, within the area unless otherwise noted.

Gaging station is a particular site on a stream, canal, lake, or reservoir where systematic observations of gage height or discharge are obtained. When used in connection with a discharge record, the term is applied only to those gaging stations where a continuous record of discharge is computed.

Hardness of water is a physical-chemical characteristic attributable to the presence of alkaline earths (principally calcium and magnesium) and is expressed as equivalent calcium carbonate $\left(\mathrm{CaCO}_{3}\right)$.

Micrograms per liter ( $\mathrm{g} / \mathrm{L}, \mathrm{UG} / \mathrm{L}$ ) is a unit expressing the concentration of chemical constituents in a sample as the mass (micrograms) of constituent per unit volume (liter) of sample. One thousand micrograms per liter is equivalent to one milligram per liter (see below).

Milligrams per liter (mg/L, MG/L) is a unit for expressing the concentration of chemical constituents in solution. Milligrams per liter represents the weight of solute per unit volume of water. Milligrams or micrograms per liter may be converted to milliequivalents (one thousandth of a gram-equivalent weight of a constituent) per liter by multiplying by the factors in the table below. Concentration of suspended sediment expressed in milligrams per liter is based on the weight of sediment in a liter of water-sediment mixture.

\begin{tabular}{lllr}
\hline \multicolumn{1}{c}{ Ion } & $\begin{array}{c}\text { Multiply } \\
\text { by }\end{array}$ & \multicolumn{1}{c}{ lon } & $\begin{array}{c}\text { Multiply } \\
\text { by }\end{array}$ \\
\hline Aluminum $(\mathrm{Al}+3)$ & 0.11119 & Iron $\left(\mathrm{Fe}^{+3}\right)$ & 0.05372 \\
Ammonia as $\mathrm{NH}_{4}+1$ & .05544 & Lead $\left(\mathrm{Pb}^{+2}\right)$ & .00965 \\
Bicarbonate $\left(\mathrm{HCO}_{3}-1\right)$ & .01639 & Lithium $\left(\mathrm{Li}^{+1}\right)$ & .14411 \\
Calcium $\left(\mathrm{Ca}^{+2}\right)$ & .04990 & Magnesium $\left(\mathrm{Mg}^{+2}\right)$ & .08226 \\
Carbonate $\left(\mathrm{CO}_{3}-2\right)$ & .03333 & Manganese $\left(\mathrm{Mn}^{+2}\right)$ & .03640 \\
Chloride $\left(\mathrm{Cl}^{-1}\right)$ & .02821 & Nitrate $\left(\mathrm{NO}_{3}{ }^{-1}\right)$ & .01613 \\
Chromium $\left(\mathrm{Cr}^{+6}\right)$ & .11539 & Nitrite $\left(\mathrm{NO}_{2}{ }^{-1}\right)$ & .02174 \\
Cobalt $(\mathrm{Co}+2)$ & .03394 & Potassium $\left(\mathrm{K}^{+1}\right)$ & .02557 \\
Copper $\left(\mathrm{Cu}^{+2}\right)$ & .03148 & Sodium $\left(\mathrm{Na}^{+1}\right)$ & .04350 \\
Fluoride $\left(\mathrm{F}^{-1}\right)$ & .05264 & Strontium $\left(\mathrm{Sr}^{+2}\right)$ & .02283 \\
Hydrogen $\left(\mathrm{H}^{+1}\right)$ & .99209 & Sulfate $\left(\mathrm{SO}_{4}{ }^{-2}\right)$ & .02082 \\
Hydroxide $\left(\mathrm{OH}^{-1}\right)$ & .05880 & Zinc $(\mathrm{Zn}+2)$ & .03060 \\
\hline
\end{tabular}


National Geodetic Vertical Datum of 1929 (NGVD) is a geodetic datum derived from a general adjustment of the first order level nets of both the United States and Canada. It was formerly called "Sea Level Datum of 1929" or "mean sea level" in this series of reports. Although the datum was derived from the average sea level over a period of many years at 26 tide stations along the Atlantic, Gulf of Mexico, and "acific Coasts, it does not necessarily represent local mean sea level at any particular place.

Partial-record station is a particular site where limited streamflow or water-quality data are collected systematically over a period of years for use in hydrologic analyses.

$\mathrm{pH}$ is a symbol denoting the negative logarithm (base 10) of the hydrogen ion concentration of a solution; $\mathrm{pH}$ values range from 0 to 14-the lower the value, the more acid is the solution; i.e., the more hydrogen ions it contains.

Refusal is a drilling term indicating the depth of a drill hole at which further penetration is impossible or impractical with the equipment being used

Specific conductance is a measure of the ability of a water to conduct an electrical current and is expressed in micromhos per centimeter at $25^{\circ} \mathrm{C}$. Specific conductance is related to the type and concentration of ions in solution and can be used for estimating the dissolved-solids content of the water. Commonly, concentration of dissolved solids (in milligrams per liter) is about 65 percent of specific conductance (in micromhos per $\mathrm{cm}$ at $25^{\circ} \mathrm{C}$ ). This relation is not constant from stream to stream or from well to well, and it may even vary in the same source with changes in composition of the water.

Water year is the 12-month period that begins with October I and ends with September 30.

\section{TEMPERATURE}

Most large streams have a small diurnal temperature change, while small, shallow streams may have a daily range of several degrees and may follow closely the changes in air temperature. Some streams may be affected by waste-heat discharges. To convert temperature data shown in degrees Celsius (centigrade, ${ }^{\circ} \mathrm{C}$ ) to degrees Fahrenhe it ( $\mathrm{OF}$ ), see following table:

Temperature conversion table, degrees Celsius $\left({ }^{\circ} \mathrm{C}\right)$ to degrees Fahrenheit (OF) of $=9 / 5\left({ }^{\circ} \mathrm{C}\right)+32$ or ${ }^{\circ} \mathrm{C}=5 / 9\left({ }^{\circ} \mathrm{F}-32\right)$

\begin{tabular}{llllllll}
\hline${ }^{\circ} \mathrm{C}$ & ${ }^{\circ} \mathrm{F}$ & ${ }^{\circ} \mathrm{C}$ & ${ }^{\circ} \mathrm{F}$ & ${ }^{\circ} \mathrm{C}$ & ${ }^{\circ} \mathrm{F}$ & ${ }^{\circ} \mathrm{C}$ & ${ }^{\circ} \mathrm{F}$ \\
\hline 0.0 & 32 & 10.0 & 50 & 20.0 & 68 & 30.0 & 86 \\
.5 & 33 & 10.5 & 51 & 20.5 & 69 & 30.5 & 87 \\
1.0 & 34 & 11.0 & 52 & 21.0 & 70 & 31.0 & 88 \\
1.5 & 35 & 11.5 & 53 & 21.5 & 71 & 31.5 & 89 \\
2.0 & 36 & 12.0 & 54 & 22.0 & 72 & 32.0 & 90 \\
3.0 & 37 & 13.0 & 55 & 23.0 & 73 & 33.0 & 91 \\
3.5 & 38 & 13.5 & 56 & 23.5 & 74 & 33.5 & 92 \\
4.0 & 39 & 14.0 & 57 & 24.0 & 75 & 34.0 & 93 \\
4.5 & 40 & 14.5 & 58 & 24.5 & 76 & 34.5 & 94 \\
5.0 & 41 & 15.0 & 59 & 25.0 & 77 & 35.0 & 95 \\
5.5 & 42 & 15.5 & 60 & 25.5 & 78 & 35.5 & 96 \\
6.0 & 43 & 16.0 & 61 & 26.0 & 79 & 36.0 & 97 \\
6.5 & 44 & 16.5 & 62 & 26.5 & 80 & 36.5 & 98 \\
7.0 & 45 & 17.0 & 63 & 27.0 & 81 & 37.0 & 99 \\
8.0 & 46 & 18.0 & 64 & 28.0 & 82 & 38.0 & 100 \\
8.5 & 47 & 18.5 & 65 & 28.5 & 83 & 38.5 & 101 \\
9.0 & 48 & 19.0 & 66 & 29.0 & 84 & 39.0 & 102 \\
9.5 & 49 & 19.5 & 67 & 29.5 & 85 & 39.5 & 103 \\
\hline
\end{tabular}




\section{CONVERSION FACTORS}

The following table may be used to convert inch-pound units to International System of Units (SI).

Multiply inch-pound units By To obtain SI Units

\begin{tabular}{|c|c|c|}
\hline \multicolumn{3}{|c|}{ Length } \\
\hline inch & $\begin{array}{l}25.4 \\
0.0254\end{array}$ & $\begin{array}{l}\text { millimeter }(\mathrm{mm}) \\
\text { meter }(\mathrm{m})\end{array}$ \\
\hline foot & 0.3048 & meter $(\mathrm{m})$ \\
\hline yard & 0.9144 & meter $(m)$ \\
\hline rod & 5.0292 & meter (m) \\
\hline mile & 1.609 & kilometer $(\mathrm{km})$ \\
\hline \multicolumn{3}{|c|}{ Area } \\
\hline square mile $\left(m i^{2}\right)$ & 2.590 & square kilometer $\left(\mathrm{km}^{2}\right)$ \\
\hline \multicolumn{3}{|c|}{ Flow } \\
\hline $\begin{array}{l}\text { cubic foot per second } \\
(\mathrm{ft} 3 / \mathrm{s})\end{array}$ & $\begin{array}{l}28.32 \\
28.32 \\
0.02832\end{array}$ & $\begin{array}{l}\text { liter per second }(\mathrm{L} / \mathrm{s}) \\
\text { cubic decimeter per second }\left(\mathrm{dm}^{3} / \mathrm{s}\right) \\
\text { cubic meter per second }\left(\mathrm{m}^{3} / \mathrm{s}\right)\end{array}$ \\
\hline gallon per minute (gal/min) & $\begin{array}{l}0.06309 \\
0.06309 \\
6.309 \times 10^{-5}\end{array}$ & $\begin{array}{l}\text { liter per second }(\mathrm{L} / \mathrm{s}) \\
\text { cubic decimeter per second }\left(\mathrm{dm}^{3} / \mathrm{s}\right) \\
\text { cubic meter per second }\left(\mathrm{m}^{3} / \mathrm{s}\right)\end{array}$ \\
\hline million gallons per day (Mgal/d & d) $\begin{array}{l}43.81 \\
0.04381\end{array}$ & $\begin{array}{l}\text { cubic decimeter per second }\left(\mathrm{dm}^{3} / \mathrm{s}\right) \\
\text { cubic meter per second }\left(\mathrm{m}^{3} / \mathrm{s}\right)\end{array}$ \\
\hline \multicolumn{3}{|c|}{ Volume } \\
\hline gallon (gal) & $\begin{array}{l}3.785 \\
3.785 \\
0.003785\end{array}$ & $\begin{array}{l}\text { liter }(L) \\
\text { cubic decimeter }\left(\mathrm{dm}^{3}\right) \\
\text { cubic meter }\left(\mathrm{m}^{3}\right)\end{array}$ \\
\hline cubic foot $\left(\mathrm{ft}^{3}\right)$ & $\begin{array}{l}28.32 \\
0.02832\end{array}$ & $\begin{array}{l}\text { cubic decimeter }\left(\mathrm{dm}^{3}\right) \\
\text { cubic meter }\left(\mathrm{m}^{3}\right)\end{array}$ \\
\hline $\begin{array}{l}\text { cubic foot per second } \\
\left.\text { per day } L\left(\mathrm{ft}^{3} / \mathrm{s}\right) / \mathrm{d}\right]\end{array}$ & $\begin{array}{r}2447 \\
0.002447\end{array}$ & $\begin{array}{l}\text { cubic meter }\left(\mathrm{m}^{3}\right) \\
\text { cubic hectometer }\left(\mathrm{hm}^{3}\right)\end{array}$ \\
\hline
\end{tabular}




\section{SELECTED REFERENCES}

Hazen, Allen, 1892, A new color standard for natural waters: American Chemical Journal, v. 12, p. 427-428.

Hem, J. D., 1970, Study and interpretation of the chemical characteristics of natural water; U.S. Geological Survey Water-Supply Paper 1473, 363 p., 2d ed.

Langbein, W. B., and Iseri, K. T., 1960, General introduction and hydrologic definitions: U.S. Geological Survey Water-Supply Paper 154!-A, 29 p.

Maevsky, Anthony, 1976, Ground-water levels in Massachusetts, 1936-74: U.S. Geological Survey open-file report, Massachusetts Hydrologic-Data Report No. 17, 107 p.

Maevsky, Anthony, and Drake, J. A., 1963, Southeastern Massachusetts: U.S. Geological Survey open-file report, Massachusetts Basic-Data Report No. 7, 55 p.

Massachusetts Department of Public Health, 1970, (and annually in previous years), Report of routine chemical and physical analyses of public water supplies in Massachusetts, 1970: Boston, Mass., Division of Environmental Health.

U.S. Geological Survey, 1954, Compilation of records of surface waters of the United States through September 1950, part I-A, North Atlantic slope basins, Maine to Connecticut: U.S. Geological Survey Water-Supply Paper I301, 380 p., I pl.

1964, Compilation of records of surface waters of the United States, October 1950 to September 1960, part 1-A, North Atlantic slope basins, Maine to Connecticut: U.S. Geological Survey Water-Supply Paper 1721, 317 p., I pl.

1967, Water resources data for Massachusetts, New Hampshire, Rhode Island, Vermont, 1966: Boston, Mass., Water Resources Division, 291 p., I pl.

1968, Water resources data for Massachusetts, New Hampshire, Rhode Island, Vermont, 1967: Boston, Mass., Water Resources Division, 305 p., I pl.

1969a, Surface water supply of the United States, 1961-65, part 1, North Atlantic slope basins, v. 1, Basins from Maine to Connecticut: U.S. Geological Survey Water-Supply Paper 1901, 1027 p., I pl.

1969b, Water resources data for Massachusetts, New Hampshire, Rhode Island, Vermont, 1968: Boston, Mass., Water Resources Division, 305 p., I pl.

1970, Water resources data for Massachusetts, New Hampshire, Rhode Island, Vermont, 7969: Boston, Mass., Water Resources Division, 347 p., I pl.

1971, Water resources data for Massachusetts, New Hampshire, Rhode Island, Vermont, 7970: Boston, Mass., Water Resources Division, 373 p., I pl.

1973, Water resources data for Massachusetts, New Hampshire, Rhode Island, Vermont,

7971: Boston, Mass., Water Resources Division, 401 p., I pl.

1974, Water resources data for Massachusetts, New Hampshire, Rhode Island, Vermont, 1972: Boston, Mass., Water Resources Division, 394 p., I pl.

1975a, Surface water supply of the United States, 1966-70, part 1, North Atlantic slope basins, Maine to Connecticut: U.S. Geological Survey Water-Supply Paper 2101, I, $123 \mathrm{p}$. 
U.S. Geological Survey, 1975b, Water resources data for Massachusetts, New Hampshire, Rhode Island, Vermont, 1973: Boston, Mass., Water Resources Division, 433 p., I pl.

1975c, Water resources data for Massachusetts, New Hampshire, Rhode Island, Vermont, 7974: Boston, Mass., Water Resources Division, 429 p., I pl.

1976, Water resources data for Massachusetts and Rhode Island, MA-RI 75-I: Boston, Mass., U.S. Geological Survey Water-Data Report MA-RI-75-1, 288 p.

1977, Water resources data for Massachusetts and Rhode Island, MA-RI 76-I: Boston, Mass., U.S. Geological Survey Water-Data Report MA-RI-76-I, 300 p.

1978, Water resources data for Massachusetts and Rhode Island, MA-RI 77-I: Boston, Mass., U.S. Geological Survey Water-Data Report MA-RI-77-1, 304 p.

1979, Water resources data for Massachusetts and Rhode Island, MA-RI 78-I: Boston, Mass., U.S. Geological Survey Water-Data Report MA-RI-78-1, 310 p.

1980, Water resources data for Massachusetts and Rhode Island, MA-RI 79-1: Boston, Mass., U.S. Geological Survey Water-Data Report MA-RI-79-I, 349 p.

1981, Water resources data for Massachusetts and Rhode Island, MA-RI 80-I: Boston, Mass., U.S. Geological Survey Water-Data Report MA-RI-80-I, 348 p.

Willey, R. E., Williams, J. R., and Tasker, G. D., 1978, Water resources of the coastal drainage basins of southeastern Massachusetts, Westport River, Westport to Seekonk: U. S. Geological Survey Hydrologic Investigations Atlas 275.

Williams, J. R., 1968, Availability of ground water in the northern part Tenmile and Taunton River basins, southeastern Massachusetts: U.S. Geological Survey Hydrologic Investigations Atlas 300

Williams, J. R., Farrell, D. F., and Willey, R. E., 1973, Water resources of the Taunton River basin, southeastern Massachusetts: U.S. Geological Survey Hydrologic Investigations Atlas 460.

Williams, J. R., and Tasker, G. D., 1978, Water resources of the coastal drainage basins of southeastern Massachusetts, northwest shore of Buzzards Bay: U.S. Geological Survey Hydrologic Investigations Atlas 560.

Williams, J. R., and Willey, R. E., 1967, Northern part Tenmile and Taunton River basins: U.S. Geological Survey open-file report, Massachusetts Basic-Data Report No. 10, 56 p.

1970, Taunton River basin: U.S. Geological Survey open-file report, Massachusetts Basic-Data Report No. 12, 102 p.

1973, Bedrock topography and texture of unconsolidated deposits, Taunton River basin, southeastern Massachusetts: U.S. Geological Survey Miscellaneous Geologic Investigations, Map 1-742.

Williams, J. R., Willey, R. E., and Tasker, G. D., 1980, Hydrologic data of the coastal drainage basins of southeastern Massachusetts, northwest shore of Buzzards Bay: U.S. Geological Survey open-file report, Massachusetts Hydrologic-Data Report 20, 30 p., I pl. 
LOCAL WELL NUMBER: LETTER PREFIX INOICATES--A, U.S. GEOLOGICAL SURVEY AUGER BORING; B, BRIOGE BORING; R, ROAOWAY BORING; W, WELL OR TEST WELL (THE "W" IS OMITTED FROM PLATE 1 TO CONSERVE SPACE); $X$, MISCELLANEOUS TEST BORING.

LATITUDE-LONGITUDE: NUMBER FOLLOWING DECIMAL POINT IS A SEQUENTIAL NUMBER FOR WELLS OR BORINGS IN A 1 -SECONO GRID.

ALTITUDE OF LAND-SURFACE DATUM: ALTITUOES ARE EXPRESSEO IN FEET ABOVE MEAN SEA LEVEL; THOSE PRECEDED BY A MINUS SIGN ARE BELOW MEAN SEA LEVEL.

METHOO DRILLED: A, AIR-ROTARY; B, BORED OR AUGERED; C, CABLE TOOL; D, OUG; H, HYDRAULIC-ROTARY; J, JETTED; P, AIRPERCUSSION; R, REVERSE-ROTARY; T, TRENCHED; V, DRIVEN; W, DRIVE-WASH.

WELL FINISH: C, POROUS CONCRETE; F, GRAVEL WALL WITH PERFORATED OR SLOTTED CASING; G, GRAVEL WALL WITH COMMERCIAL SCREEN; H, HORIZONTAL GALLERY OR COLLECTOR; O, OPEN END; P, PERFORATED OR SLOTTED CASING; S, SCREEN; T, SANO POINT; W, WALLED OR SHORED; $X$, OPEN HOLE IN AQUIFER (GENERALLY CASED TO AQUIFER).

WELL OEPTH: DEPTH OF FINISHED WELL, IN FEET BELOW LAND SURFACE.

WELL USE: A, ANODE; D, DRAINAGE; G, SEISMIC HOLE; H, HEAT RESERVOIR; 0, OBSERVATION; P, OIL OR GAS; R, RECHARGE; T, TEST; U, UNUSED; W, WATER WITHDRAWAL; $X$, WASTE DISPOSAL; $Z$, DESTROYED.

WATER-BEARING MATERIAL: PRINCIPAL WATER-BEARING ZONE.
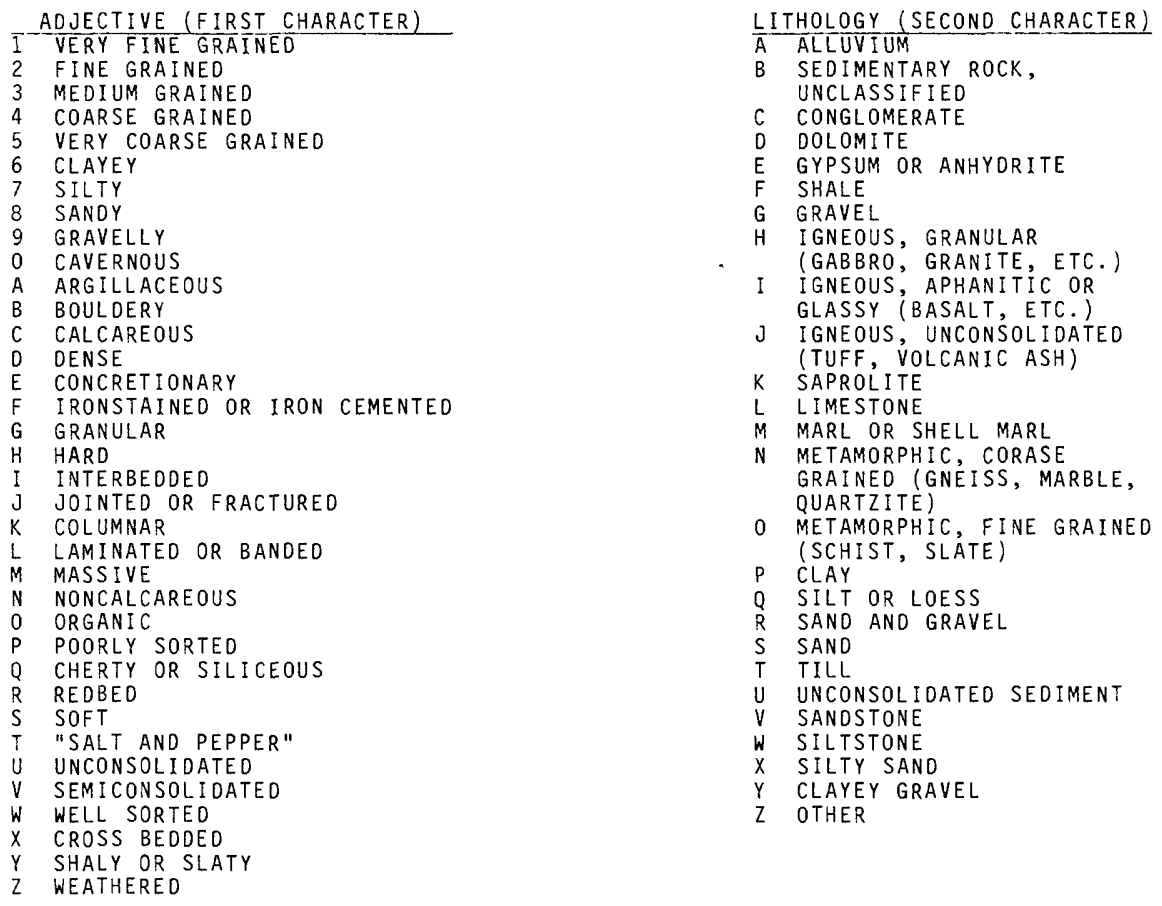

WATER LEVEL: LEVELS ARE GIVEN IN FEET BELOW LANO SURFACE; "+ INOICATES WATER LEVEL ABOVE LANO SURFACE; "F" INOICATES FLOWING WELL.

WATER USE: A, AIR CONDITIONING; B, BOTTLING; C, COMMERICAL; D, OEWATERING; E, POWER GENERATION; F, FIRE PROTECTION;

H, DOMESTIC; I, IRRIGATION; M, MEOICINAL; N, INOUSTRIAL (INCLUOES MINING); P, PUBLIC SUPPLY; R, RECREATION; S, STOCK; T, INSTITUTIONAL; U, UNUSEO; V, REPRESSURIZATION; W, RECHARGE; $X$, DESALINATION--PUBLIC SUPPLIES; Y, DESALINATION--OTHER

PUMPAGE/YIELD: IN GALLONS PER MINUTE (GAL/MIN).

PUMPAGE/ORAWDOWN: THE DIFFERENCE BETWEEN STATIC WATER LEVEL AND PUMPING LEVEL.

PUMPAGE/TIME: THE FOLLOWING CODES ARE USED FOR PUMPING PERIODS OF LESS THAN 1 HOUR: A, THROUGH 15 MINUTES; B, 15 TO 30 MINUTES; $C, 30$ T0 45 MINUTES; 0,45 TO 59 MINUTES.

LOG: D, DRILLER'S LOG; E, ELECTRIC LOG; G, GEOLOGIST'S LOG AVAILABLE IN TABLE 2.

QW: TYPE OF CHEMICAL ANALYSIS AVAILABLE IN TABLE 3. C, COMPLETE; J, CONOUCTANCE ANO CHLORIDE; K, CONDUCTANCE;

L, CHLORIDE; M, MULTIPLE (INCLUDES ONE COMPLETE AND ONE OR MORE PARTIAL); P, PARTIAL. 


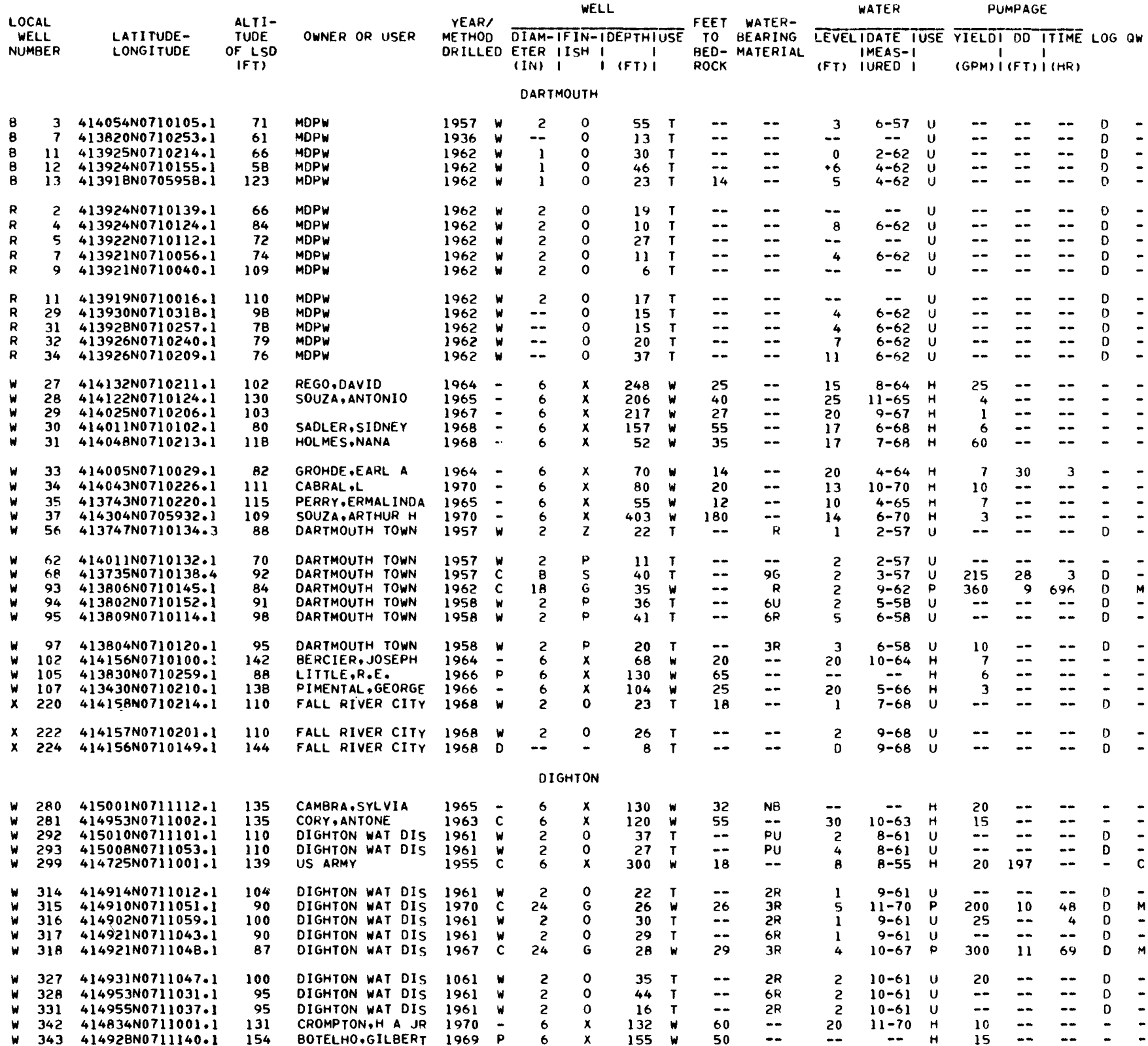

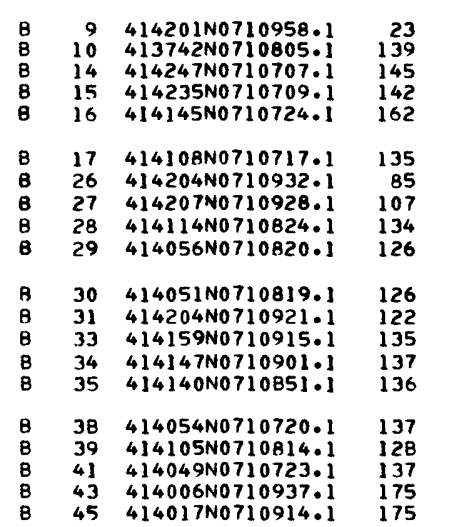

FALL RIVER

\begin{tabular}{|c|c|}
\hline $\begin{array}{l}\text { MDPW } \\
\text { MDPW } \\
\text { MDPW } \\
\text { MDPW } \\
\text { MDPW }\end{array}$ & $\begin{array}{l}1949 \\
1938 \\
1955 \\
1954 \\
1954\end{array}$ \\
\hline $\begin{array}{l}\text { MDPW } \\
\text { MDPW } \\
\text { MDPW } \\
\text { MDPW } \\
\text { MDPW }\end{array}$ & $\begin{array}{l}1955 \\
1957 \\
1959 \\
1962 \\
1962\end{array}$ \\
\hline $\begin{array}{l}\text { MDPW } \\
\text { MDPW } \\
\text { MDPW } \\
\text { MDPW } \\
\text { MDPW }\end{array}$ & $\begin{array}{l}1962 \\
1959 \\
1959 \\
1959 \\
1962\end{array}$ \\
\hline $\begin{array}{l}\text { MOPW } \\
\text { MDPW } \\
\text { MDPW } \\
\text { MDPW } \\
\text { MDPW }\end{array}$ & $\begin{array}{l}1959 \\
1962 \\
1959 \\
1962 \\
1962\end{array}$ \\
\hline
\end{tabular}

\section{$-$}

$\begin{array}{ll}2 & 0 \\ 2 & 0 \\ 2 & 0 \\ 2 & 0 \\ 2 & 0 \\ 2 & 0 \\ 2 & 0 \\ 2 & 0 \\ 2 & 0 \\ 2 & 0 \\ 2 & 0 \\ 2 & 0 \\ 2 & 0 \\ 2 & 0 \\ 2 & 0 \\ 2 & 0 \\ 2 & 0 \\ 2 & 0 \\ 2 & 0 \\ 2 & 0\end{array}$


LOCAL WELL

LATITUDELONGITUDE
ALT I OF LSD (FT)

WELL

OWNER OR USER METHOD DIAM-IFIN-IOEPTHIUSE FO BEARING

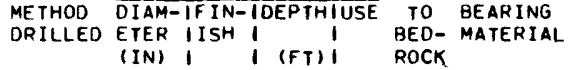

WATEP

PUMPAGE

FALL RIVER --CONTINUED

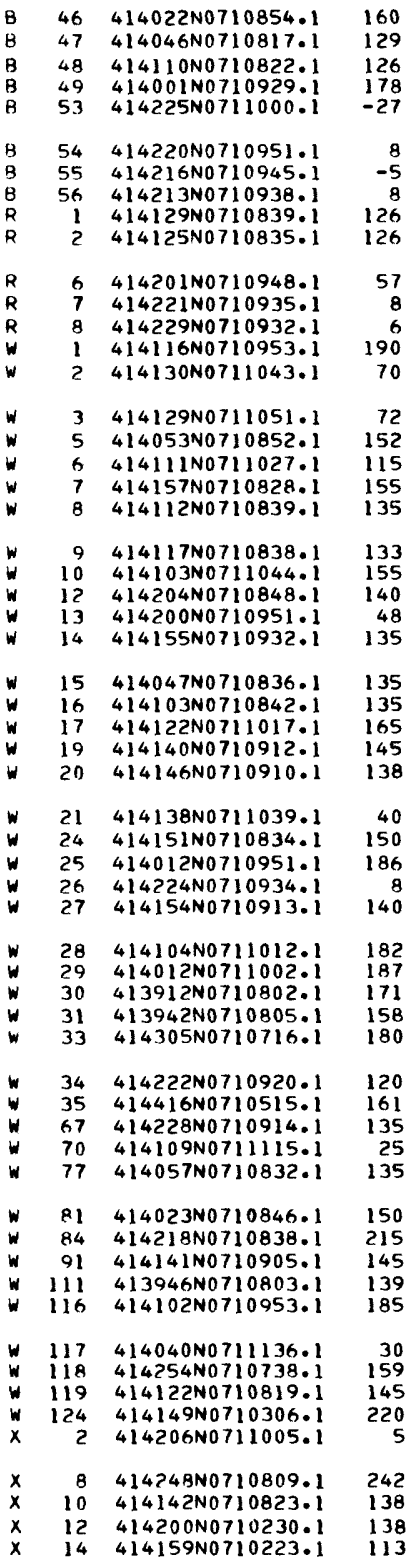

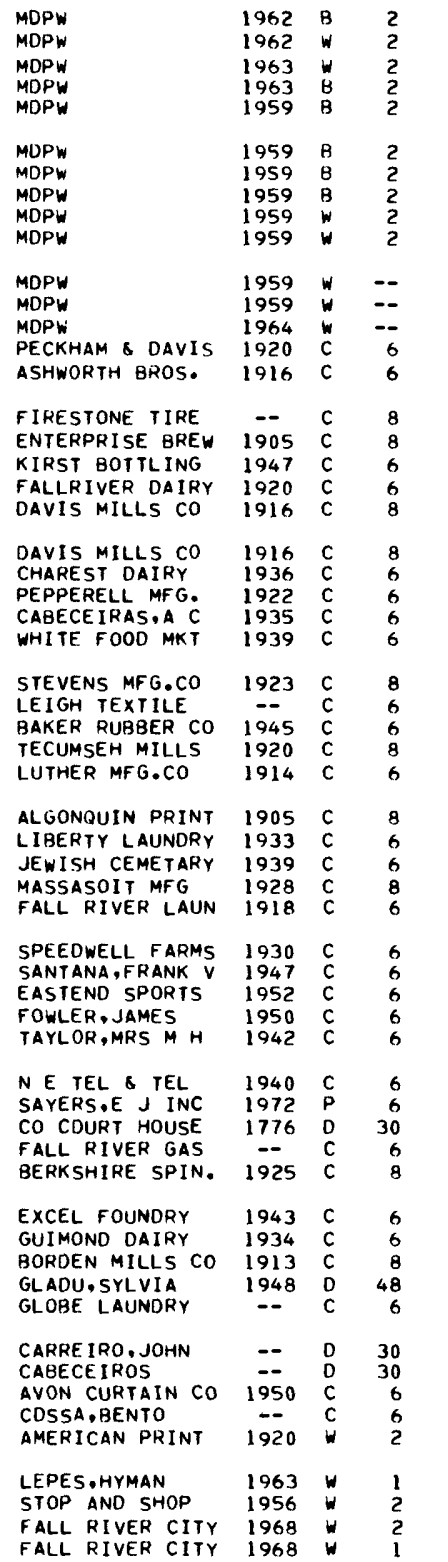

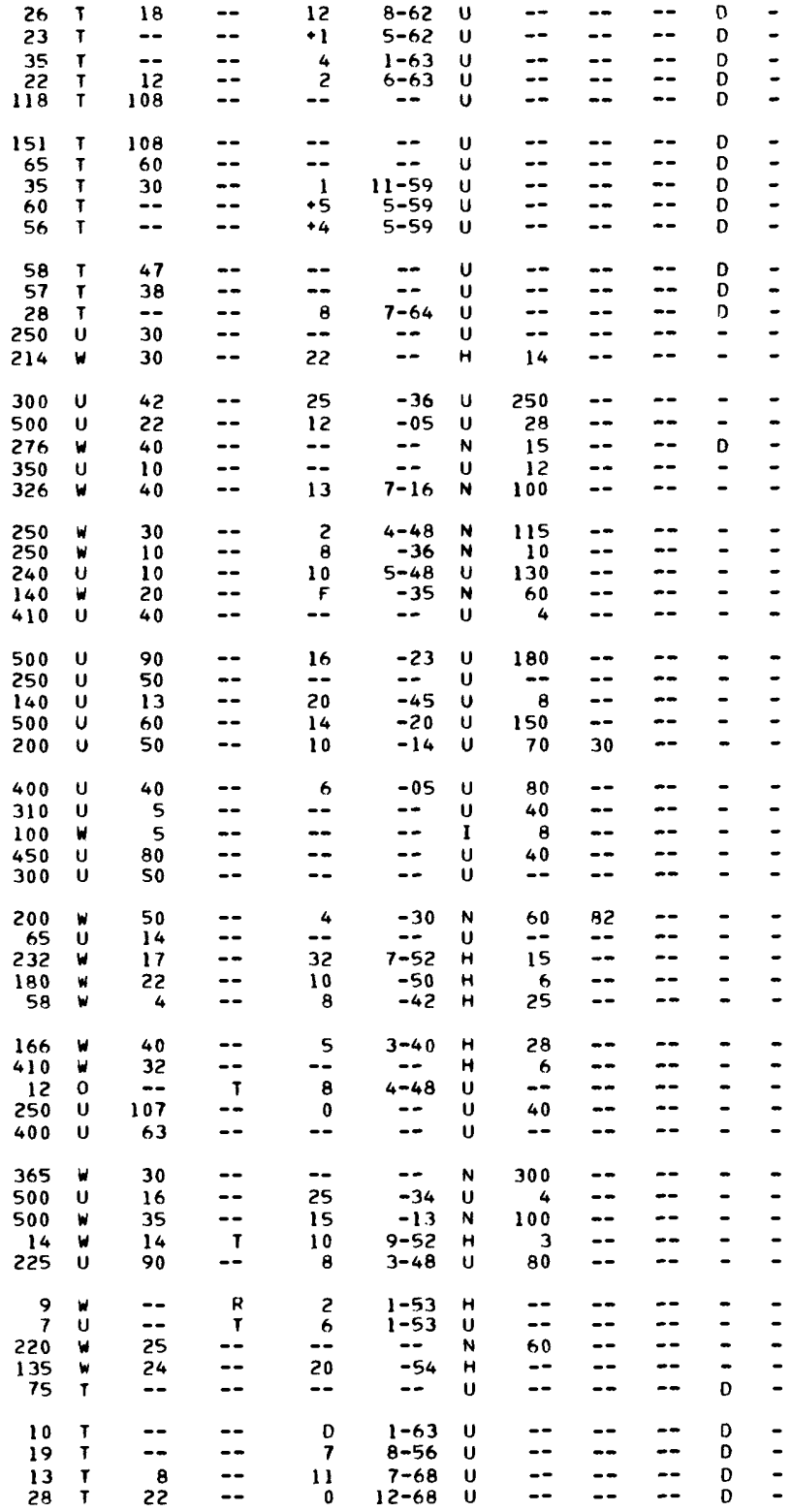

FREE TOWN

$1414833 \mathrm{NO} 711642.1$ 2414631 No711653. 4 415030NOP11441. 5 415032N0711434.1

6 414R01NOT11624. $7414653 N 0711718.1$ R 414642N0711647. Q $414640 \mathrm{~N} N 711634.1$
I $414653 \mathrm{~N} 0711512.1$

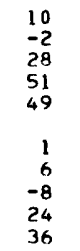

MDPW

MDPW

MDPW

MDPW

MDPW

MOPW

ORMAND, FRANK

\section{4 - 6 X}

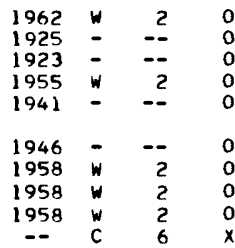

$100 W 50$

$\begin{array}{rrrr}25 & T & -- & -- \\ 33 & T & -- & -- \\ 14 & T & -- & -- \\ 25 & 1 & -- & -- \\ 26 & 1 & -- & - \\ 42 & T & -- & -- \\ 141 & 1 & 136 & -- \\ 28 & T & -- & -- \\ 59 & T & 54 & -- \\ 98 & W & -- & -\end{array}$
LEVELIDATE IUSE YIELOI DO ITIME LOG OW (FT) IURED I

(GPM)Í(FT) I (HR) 
LOCAL WELL
LATITUDELONGI IUDE
ALTITUDE OWNER OR USER (FT)
WELL YEAR/ TIAM-IFIN-IDEPTHIUSE FEET WATERDRILLED ETER IISH I (IN) I I IFTII

REHOBOTH --CONTINUEO$$
\begin{array}{lrl}
w & 2 & 414644 N 0711532.1 \\
W & 3 & 414644 N 0711549.1 \\
W & 4 & 414645 N 0711603.1 \\
w & 5 & 414648 N 0711619.1 \\
W & 6 & 414638 N 0711635.1 \\
W & 7 & 414713 N 0711634.1 \\
W & 8 & 414747 N 0711609.1 \\
W & 9 & 414720 N 0711631.1 \\
W & 10 & 414658 N 0711629.1 \\
W & 12 & 414635 N 0711720.1
\end{array}
$$

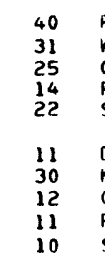

READ, R B WYMAN FARM
CARDOZA.M CARDOZA,M SILVA,A

DIAS,OIAGO KIMBALL. I RLOBUS $F A R$

SUTCLIFFE, G

$\begin{array}{cc}1950 & C \\ -- & 0 \\ -\because & D \\ 1-- & D \\ 1940 & C \\ -- & 0 \\ 1938 & C \\ 1930 & C \\ 1933 & C \\ 1947 & C\end{array}$

$14 \quad 414645 N 0711729.1$

$15 \quad 414708$ N0711713.1

$16 \quad 414719 \mathrm{N0711705.1}$

19 414752N0711737.1

22 414749N0711542.1

24 414804 NOT11620.

$\begin{array}{ll}26 & 414838 \text { No711739.1 } \\ 27 & 414830 \text { No711717.1 }\end{array}$

$28 \quad 414815$ N0711702.1 29
30 414811 N0711646.1

30414809 NO711640.

31
32 $414818 \mathrm{~N} 0711737.1$

12

HATHAWAY,H

ALEMEDIA, A

SANOBERG, $C$ J

THOMAS $5 E A$ FOOD

$\begin{array}{lll}-- & 0 & 30 \\ -- & 0 & 30\end{array}$

PRAMAS $C$ W JR
PRA FOOD

1935

CLARK, HARRY P

DUARTE, OAVID

COSTA,JOSEPH

PIMENTAL .A

COUGHLIN, ROBERT

1951

REEO, JOHN L

HASS, JOSEPH $P$

HASS, JOSEPH P

ESTRELLA.ANTONE I951

$\begin{array}{lll} & \\ \text { OLIVERA,FRANK } & 1951 & \mathrm{C}\end{array}$

$33 \quad 414833 N 0711532.1$

34 414836N0711553.

$36 \quad 414820$ NO711610.

$38 \quad 414807 \mathrm{~N} 0711534$.

40 414833N0711538.1

41 414826N0711543.

$42 \quad 414907 N 0711524$.

$44 \quad 414933 \mathrm{~N} 0711528.1$

$45414947 \mathrm{~N} 0711520.1$

$47 \quad 414839 N 0711707$.

$49 \quad 14858 N 0711709$

$54 \quad 414843 N 0711639.1$

$57414907 N 0711624.1$

58 414905N0711556.1

62 414937N0711601.

$64 \quad 414949 \mathrm{~N} 0711545.1$

$66415409 \mathrm{~N} 0711559 \cdot 1$

$67 \quad 4154$

$68 \quad 415336 \mathrm{~N} 0711617$.

70 415302NO711652.1

$71415003 \mathrm{~N} 0711642.1$

$73415004 \mathrm{~N} 0711506$.

$74 \quad 415014 N 0711505.1$

75
77 $415339 \mathrm{~N} 0711612.1$

78 415028N0711509.1

$79415033 \mathrm{~N} 0711501.1$

80 415027N0711526.1

81
82

$83415042 N 0711527.1$

85 415040N0711532.1

$86 \quad 415021 N 0711627.1$

$415353 N 0711558.1$

89 415127N0711510.1

90 415114N0711516.1

91415350 N0711552.1

$92415143 \mathrm{~N} 0711521.1$

94 415202NOT11530.

$954152111 N 0711539$.

96414645 N0711725.1

97 415221N0711620.1

98
100 $415217 \mathrm{~N} 0711619.1$

\section{TRAVIS,MARY}

SANTOS, MANUEL

TOWNSEND, A $S$

COLBETH, ARCHIE

NAJARIAN GEORGE

ABRAMS, C
MELLL, JOSEPH

MELLLN,JOS C

RAMSPOTT ROBERT

RAMSPOTT.ROBERT

NOONAN, EOWAR

CHAPPELL, H B

MANZIGAN, SURIA

$\overline{1932}$

1940

$\ddot{910}$

WHITE, JOHN

KINOBERG, VIMER

OESOUZA, JAMES

OEMERS,ROYAL T

YOUNG,LEO

DALTON, WILFRED

PETZOLO, PAUL

LONG,RUSSELL E 1963

ANTHONY,AGNES 1952

ANTHONY, AGNES

BURNETT,A C

DUNN, WILLIAM

$\begin{array}{cc}1952 & C \\ - & 0 \\ - & C \\ 1965 & P \\ -- & 0\end{array}$

HORTON FARM

PORC.ENAMEL CO

BULLOCK, HARR IS

LOVETT, CHARLES 1950

REHOBOTH EL SCH 1951

MC CLELLLANO, ANN

STATE POL ICE

WARREN, ALBERT

FERRINI, JOSEPH

1942

1944

JASTRAM,E $P$

MAKER, WINSTON

HATHAWAY OLLOYD

GOFF, HAROLO A

$1949 \mathrm{C}$

1949
1965
--

APPLEBY,L E

ARNAUSKAS, 8

$\begin{array}{lll}\text { SWANSON,GEORGE } & -- & 0 \\ \text { GILMORE, S H } & 1948 & \mathrm{C} \\ \text { ALLEN, GEORGE N } & 1910 & \mathrm{C}\end{array}$

$\begin{array}{lcc}\text { SWANSON,GEORGE } & -\overline{0} & 0 \\ \text { GILMORE,S H } & 1948 & \mathrm{C} \\ \text { ALLEN,GEORGE N } & 1910 & \mathrm{C}\end{array}$
NAJARIAN, GEORGE

KINNIE'S DAIRY 1941 C
BURRY,ALEERT JR 1956 P

FISHER, MARSHAL
WATER

PUMPAGE

LEVEL IOATE IUSE YIELOT DO ITIME LOG OW (FT) IURED

(GPM) I (FT) I (HR) 
LOCAL

WULL
ALT I TUDE OF LSD
W $101415117 \mathrm{~N} 0711557.1$ $415058 \times 0711546.1$ $104 \quad 414855 N 0711641.2$ W $106 \quad 415155 N 0711716.1$

W $108 \quad 415128 N 0711724.1 \quad 220$ w 109 41510SNO711716.1 140 W 110 415043N0711710. W $112 \quad 415023 N 0711713.1$

W $114415059 N 0711637.1$ $116 \quad 414940 N 0711559.1$ W $118 \quad 415124 N 0711714.1$ W 120415050 N0711730.1

w 121 414948N0711719.1 $w 123415000 N 0711527$. W $124 \quad 415030 N 0711731$. W $125415024 N 0711654.1$ $126415022 N 0711645.1$

W $127 \quad 415050$ N0711602.1 W $129 \quad 415040 N 0711617.1$ W $130415019 N 0711413.1$ W 132 414925N0711330.1

w 133415006 N0711713.1 w $134 \quad 415009 N 0711700.1$ W $137414835 N 0711448.1$ w $140 \quad 415129 N 0711400.1$

w 141 415213N0711404.1 $142415333 N 0711745$. $143415342 N 0711711$. $144415351 N 0711700$. $145415355 N 0711644.1$

W $146 \quad 415356 N 0711636.1$ W $147 \quad 415418 \mathrm{~N} 0711346.1$ W $148 \quad 415350 N 0711319.1$ 149
$w \quad 152$
$w 14319$

w $154 \quad 414836$ N0711644.1 W 156415238 N0711237.1 w 157 415250N0711236.1 $159415334 N 0711148.1$

W 161415401 N0711222.1 $163415413 N 0711403.1$ $164415332 N 0711712.1$ 165415331 N0711711.1 $166415241 N 0711534.1$

W $167 \quad 415237$ N0711709.1 I68 $415253 N 0711624.1$ IRB 415253N0711624.1 $\begin{array}{lll}169 & 415247 N 0711623.1 & 175\end{array}$ w 177 415225N0711756.1 202

W $179414741 N 0711706.2 \quad 20$ W 180 415121N0711658.1 170 $183414953 N 0711627.1$ $184 \quad 414805 N 0711611.1$

W 185414815 N0711605.1 W 186415038 N0711621.1 W 18714904 N0711635.1 188 415037N0711736.1 188
$W \quad 189$ 415951N0711545.1

W $190 \quad 414959$ N0711520.1 192414840 NOT11633.1 $193415225 N 0711609.1$

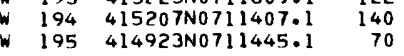

W $196 \quad 414927 N 0711442.1 \quad 69$ W 197 414951N0711340.1 $198415043 \mathrm{~N} 0711305.1$ $199 \quad 415149 N 0711441.1$
$W 01415116 N 0711424.1$

\section{60
55
200
10
220
140
110
72
60}

90

180

200
140

42

88
60

40

32
70
75
60

112

50
50
95
50
72

135
158

187
180

150

150
140

120
139

20

133
141

185

122
140

172
174

112

80

59

45
47
59

59
122

68

50

25
122$$
\text { C }
$$

WELL OWNER OR USER METHOD DRILLED DIAM-IFIN-TOEPTHIUSE BO
IIER IISH I (IN) I I I I ROCK

FEET WATER-

REHDBOTH --CONTINUED

YOUNG, JOSEPH LARSON, $5 \mathrm{M}$ PHILLIPS,W $V$ WJAR TV

PHILBRODKE, C

$\begin{array}{lccc}\text { PENKINSON,H E } & 1949 & C & \\ \text { AMORAL, W } & -- & 0 & 24 \\ \text { JENNINGS, RALPH } & 1951 & 0 & 30\end{array}$

$\begin{array}{lccc}\text { JENKINSON,HE } & -- & 0 & 24 \\ \text { AMORAL,W } & -- & 0 & 30 \\ \text { JENNINGS,RALPH } & 1951 & C & \end{array}$

RICHARDSON, W

MULL IGAN, ROBERT

KENWORTHY, H JR

FRUTADO , JOSE

BROWN,F T

VEADER,ERNEST F

UNSWDRTH, JDHN

VI ALLE, DAIRY

WEST, MARY E

KAMMERER OJ

HARRI SON,$W$

$F O X, F B$

ALLARD, ROGER U

CASTLE, HENRY W

DE MATTOS,M

DIAS, J

BENTZINGER, H A

HARRIS, HENRY D

$\begin{array}{ccr}1940 & C & 6 \\ -- & 0 & 24 \\ 1965 & P & 6 \\ 1948 & C & 6 \\ -- & D & 24 \\ 1949 & C & 6 \\ -- & 0 & 24 \\ -- & 0 & 30 \\ 1951 & C & 6 \\ -- & D & 36\end{array}$

1963

1942

1925

48
6
6
24

$1960 P$

$\begin{array}{rrr}962 & P & 6 \\ -- & 0 & 36\end{array}$

$\begin{array}{lll}-- & 0 & 36 \\ -- & D & 24\end{array}$

$1951 \mathrm{~V}$

$1914 \mathrm{C}$

1947

JACQUES,ALDERIC 1951 C

$\begin{array}{lll}\text { GENOREAU,A C } & 1964 & \text { P } \\ \text { STRDNG.LESTER W } 1955 \text { P }\end{array}$

KULIS,ALEXANDER 1963 P

SALOIS,H LINDOR 1962

LAMARRE, T

FULLER, FRANK

HAGAR, AL TON

$1965 P$

HORNBINE CHURCH -- D

BRISTOL CO WAT 1957

DZALA,MICHAEL

RICHARD, A J

TESSIER,PAUL E

TRUDELL, R N

SPELLMAN,L

KANARI AN, N

KANAR I AN, PETER

DONATO,PETER D

MELLD, THOMAS J

SWANSON, C V

CASAYANT,M T

COTE, GEDRGE

BATESON, JAMES

PORTELLA,ROBERT

DAVIS, JDHN G

DEANE, PETER $B$

DOGGETT EDWARD

JOL IN, ARMAND $P$

RASSOL. JAMES T

IRELAND, ALLEN

ABRAMS, WILLIAM

AGOPI AN, A

MAGOPIAN,A

CHURCH,HECTOR

WILSON, DUANE

CODK, RUSSELL L

BOREN,ERNEST

LEASOR, LEO F
STAPLETON,W V

$\begin{array}{rr}6 & x \\ 24 & x \\ 6 & x \\ 24 & \\ 6 & x \\ 24 & \\ 30 & x \\ 6 & x \\ 36 & \\ 48 & x \\ 6 & x \\ 6 & x \\ 24 & x \\ 6 & x \\ 6 & x \\ 6 & x \\ 36 & \\ 36 & \\ 24 & \\ 2 & y \\ 6 & x \\ 6 & x \\ 6 & x \\ 6 & x\end{array}$

$\begin{array}{rllll}75 & W & 20 & -- & = \\ 31 & W & - & -- & 28 \\ 155 & W & 80 & = & - \\ 180 & W & 25 & = & 1 \\ 18 & W & -- & -- & 13\end{array}$

$\begin{array}{rrrrr}103 & w & 8 & -- & 46 \\ 15 & w & - & = & 1 \\ 17 & w & -- & -- & 1 \\ 140 & w & 40 & -- & - \\ 24 & w & - & -- & 1\end{array}$

23

145

135
20

$125 W$

115

60

17

13

28
147

$125 W \quad 35$

$\begin{array}{rr}170 & W \\ 95 & 114 \\ & 48\end{array}$

28
55

55
160
125

$55 * 33 \quad-\quad-$

$100 W$

200

187
55

$80 \mathrm{~W}$

95

$\begin{array}{rrrrr}200 & w & 8 & -- & -- \\ 14 & W & -- & -- & 8 \\ 18 & w & -- & -- & 15 \\ 98 & W & -- & -- & --\end{array}$

28

140
110

110

140
260

140

275

160
95

125

350

122
125

410

$145 \mathrm{~W}$

163

150
215
155

$155 W$

$172 W \quad 100$
$200 * 6$

180

110

140

70
225

120

185

\begin{tabular}{r|r}
140 & $W$ \\
80 & 30 \\
185 & 25 \\
500 & 20
\end{tabular}
WATER

Pumfage

LEVEL IDATE IUSE YIELDI DD ITIME LOG QW (FT) IURED

(GPM) । (FT) ।(HR) 
LOCAL

WELL LATITUDE- ALTI LATITUDE- TUDE
LONGITUDE OF LSD OF LSO
WELL

OWNER OR USER METHOD OIAM-IFIN-IDEPTHIUSE FEET WATERMEPALD OIAM-IFIN-TOEPTHIUSE TO BEARING LEVELTDATE IUSE VIELOT DO TTIME LOG OW $\begin{array}{cllll}\text { ORILLEO ETER IISH I I I } & \text { BED- MATERIAL } \\ \text { (IN) I } & \text { I IFT) I ROCK IMEAS-I } & \text { (FT) IURED I }\end{array}$

REHOBOTH --CONTINUED

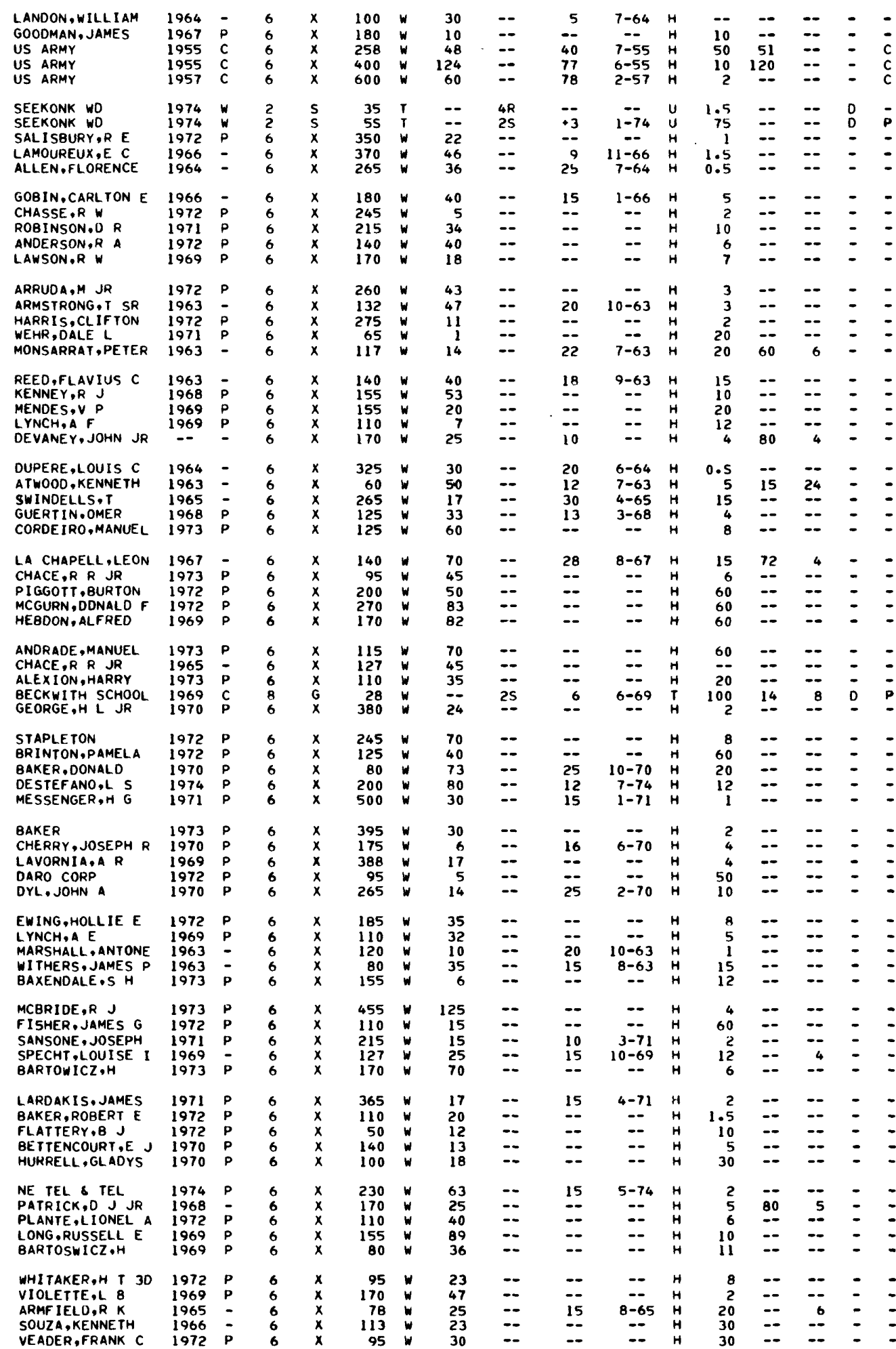


LOCAL WELL

LATITUDELONGI TUDE
ALTI TUDE OF LSO (FT)

\begin{abstract}
$295 \quad 414716$ N0711449.1 $294 \quad+14718 N 0711444.1 \quad 65$ 297
214741 N0711444.1 290
299
$414819 N 0711445: 1$

$300 \quad 414829$ N0711449.1 $304 \quad 415019 \mathrm{~N} 0711506.1$ $305 \quad 414940$ N0711603.1
$306 \quad 414951$ N0711628.1 307 415122N0711547.1

308 414823N0711608.1 $309 \quad 4 ! 4736 \mathrm{~N} 0711608.1$

$310 \quad 415232$ No711525.1

311414810 N0711453.1

313414848 N0711451.1

W $314 \quad 414737 N 0711325.1$

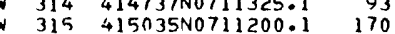
315 415151N0711208.1

317415159 N0711208.1 134 * 319 415408N0711226.1 122

w 320415411 N0711230.1 123 $\begin{array}{lll}321 & 415338 \mathrm{~N} 0711757.1 & 150 \\ 322 & 414905 \mathrm{NO} 11551.1 & 42\end{array}$ - 322 414905N0711551.1 $\begin{array}{r}324 \\ \times \quad 415119 N 0711404.1 \\ \hline\end{array}$

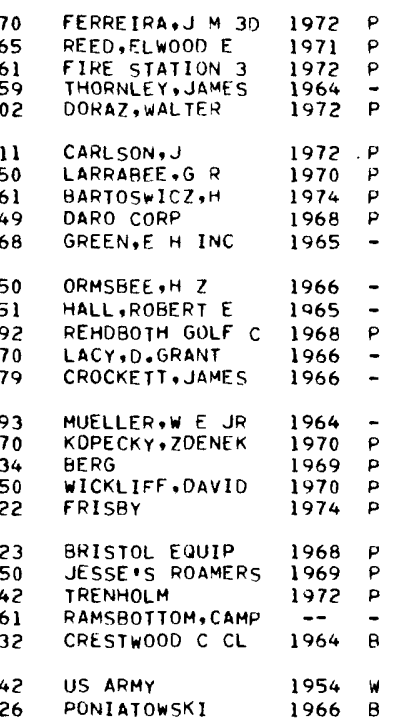

$P$

$$
\text { (IN) } 1 \text { I (FT)I }
$$

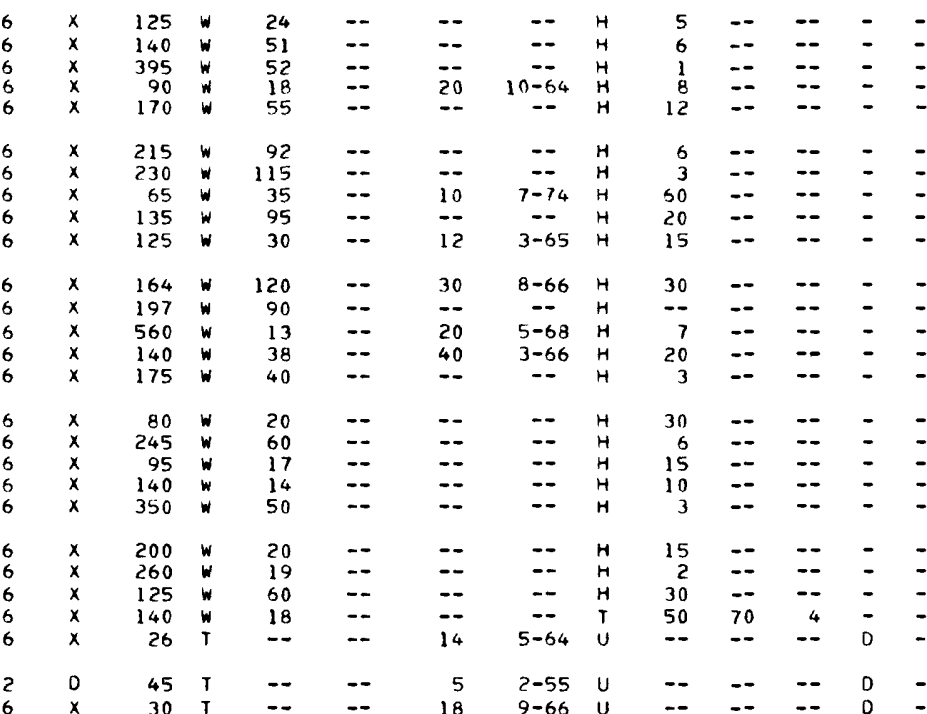

SEEKONK

MDPW

MDPW

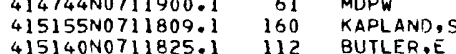

BOWLIN, ERNEST

BISHOP, $W$

WHEELER SCHOOL

CARPENTER, RUTH
MOSARRAT, $N$

$1549=$

$\begin{array}{ll}1958 & 8 \\ 1958 & 8\end{array}$

-2
2
30
24

$$
\begin{array}{r}
36 \\
84 \\
34 \\
23 \\
18 \\
11 \\
130 \\
300 \\
32 \\
180
\end{array}
$$

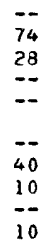

ZINDAL, R

BEEDE, ROBERT

BELL,ERNEST

CLEGG, FRANK

$\overline{1933}$

$1935 \mathrm{C}$

CLEGG,FRANK

KENT, ROBERT

$\begin{array}{lll}\text { LEVEN, IRVING } & 1967 & - \\ \text { RAU,FF } & -- & 0\end{array}$

-.

SEEKONK TOWN

FOREST HILLS 1949

BROWNING HELEN $1945 \mathrm{C}$

LEPAGE, ROLANO 1968

YAGHJIAN,A A 1944 C

CARMAN.J A

GFANT, HOLLIS E --

READ DAIRY FARM 1933 C

GOMES, JOSEPH

ADAMS, W A JR

PAPA, MARIA

MELLO, MANUEL

19200
1968

$\overline{1968}$

MT CARMEL $C H$

$1930 \mathrm{C}$

CONTO, JOSEPH

CDROE IRO, MANUEL

COSTA,LEITER M

1966

MARSHALL OFRANK

$\begin{array}{ll}1922 & C \\ 1933 & C\end{array}$

PECK FARMS

SEEKONK SWIM CL 1910

MATTA M

$\begin{array}{lll}\text { AMARAL, M } & 1930 & \text { C } \\ \text { QUATTRUCCI,A A } 1970 & -\end{array}$

$\begin{array}{rrrr}21 & w & -- & = \\ 100 & w & 10 & = \\ 212 & w & 102 & = \\ 200 & w & 55 & = \\ 15 & w & -- & --\end{array}$

$250 \mathrm{U}$

95
125
10

$\begin{array}{cccc}10 & w & -2 & 10\end{array}$

186

120

200

255 20 -- 21

$150 w$

$\begin{array}{rl}150 & w \\ 12 & w \\ 10 & u \\ 17 & u\end{array}$

$\begin{array}{rllll}17 & U & - & - & \\ 150 & w & 75 & -- & 14\end{array}$

12

145
15
110

15
110
120

155

8
60

$\begin{array}{llll}60 & = & -- & 16\end{array}$

120

15

120
75

$\begin{array}{rlll}15 & 30 & -- & 18 \\ 100 & 50 & -- & 60\end{array}$

OLIVER, A

OLIVER, JOSEPH 1945

\begin{tabular}{lcc} 
OECOSTALEITE,J & 1945 & $C$ \\
AMARAL. ANTHONY & -- & $D$ \\
\hline
\end{tabular}

$\begin{array}{llll}15 & w & -- & = \\ 78 & w & 40 & = \\ 23 & w & -- & = \\ 20 & w & -- & = \\ 67 & & 26 & --\end{array}$
WATER

PUMPAGE

VEL IDATE IUSE YIELOT DD ITIME LOG OW MEAS(GPM) \& (FT) । (HR) 
LOCAL NUELL

$$
\begin{array}{lc}
\text { ALTI- } & \\
\text { LATITUDE- } & \text { TUDE } \\
\text { LONGITUDE } & \text { OF LSD }
\end{array} \text { OWNER OR USER }
$$

WELL. METHOD DIAM-IFIN-IDEPTHIUSE FEET WATERMETHOD DIAM-IFIN-IDEPTHIUSE TO BEARING
DRILLED ETER IISH I I I BED- MATERIAL
(IN) I

WATER

PUMPAGE SEEKONK --CONTINUED

$61414851 N 0711836.1$ 62
63 $4148849 N 0711934.1$ $\begin{array}{ll}63 & 414849 N 0711923.1 \\ 65 & 414847 N 0711753.1\end{array}$ 66 414849N0711813.1

$68 \quad 414852 N 0711846.1$ $69414741 N 0711802$. 70 414841N0711948. 72 414857N0711808. 4314647 N0711753.

$\begin{array}{ll}74 & 414915 N 0711812.1 \\ 75 & 414718 N 0711940.1\end{array}$ 76 414918N0711940.1 78
$814943 N 0711809.1$
80 $414947 N 0711756.1$

84 414933N0711854.1 $87414907 N 0711839.1$ $89414922 N 0711908.1$ $90414922 N 0711918.1$

W $93 \quad 414913$ N0711901.1 W $95414959 \mathrm{N0711755.1}$ - $98415014 N 0711608$.

* $101415037 N 0711841.1$ W 103 415024N0711830. $104 \quad 415005 N 0711833.1$
$105 \quad 414950 N 0711831.1$ W 106 414945N0711848.1

W $107414942 N 0711905.1$ W 109 414740N0711949.1 W 111 414755N0711923.1 $112414951 N 0711813.1$
$W \quad 114 \quad 415054 N 0711922.1$

W $115 \quad 415052 N 0711937.1$ W $116415040 N 0711940.1$ W 119 415120N0711922.1 W 123 415001N0711853.1

W $126 \quad 415025 N 0711907.1$ 128
$W$
$w 29$ $415014 N 0711936.1$ $129415224 N 0711931.1$
$W \quad 130 \quad 415213 N 0711933.1$ $130415213 N 0711933.1$
$W \quad 132 \quad 415216 N 0712002.1$

W $135 \quad 415151$ N0711951.1 W $136425156 N 0711950.1$ W $139415135 N 0712931.1$ W 141 415115N0711943.1

W $150415042 N 0712005.1$ W $151415054 N 0711957.1$ W $152415033 \mathrm{~N} 0712017$. $W \quad 157 \quad 414950 N 0711912$. W 159 414956N0712016.1

W 160 414948N0712029.1 W 162414833 N0712015. * $163414926 \mathrm{N0711953.}$ W 166 414931N0712004.1

W $167 \quad 414928 N 0712018.1$ W 168 414900N0712010.1 W 169 414911N0712022.1 $171 \quad 414948 N 0711952.1$
$173 \quad 415227 N 0712001.1$

W $175415121 N 0711949$. W 177 414953N0711945.1 - 178 415207N0711926.1 W $179414826 N 0711949$.

W $182 \quad 414820$ N0711936.1 * 183414807 NOT11820. W 185 414804N0711814. $185414804 N 0711814.1$
$189414954 N 0711930.1$ $189414954 N 0711930.1$
$W \quad 190 \quad 415004 N 0711928.1$

142
60
54
50
70

98
32
80
75
15

MAYER,LUCAS B 1973 HICKS,PETER 1950 C 6 MELLO,CHARLES M 1974 ALPERT S SIMPSON -- D 104

COSTA, MANUEL QUEQUECHAN DEV 1973 MARSHALL, JOSEPH 1928 C $\begin{array}{lll}\text { FREDRICK5ON,O } & 1949 & \text { C } \\ \text { CABRAL,DAVID } & 1973 & \text { P }\end{array}$

MEDERIOS, A

$\begin{array}{lll}\text { READ, WALLACE } & 1964 & W \\ \text { OLIVERA,ANTHONY } & 1946 & \text { C } \\ \text { KINNEY,EDITH } & 1935 & \text { C }\end{array}$

$\begin{array}{lll}\text { KINNEY,EDITH } & 1935 & \mathrm{C} \\ \text { SANTOS,MARY } & 1935 & \mathrm{C}\end{array}$

MELVIN,E

CHAFFEE, EDGAR

AMARAL,$E U$

$\checkmark$ IERRA, JOSEPH

1951

CAMARA, JOSEPH

ARAJUO. ANTONE KNOWLES, ALBERT SANTOS, SIMON DORR , JOSEPH TURNER, $R F$

BERRY,M T

ENOS, GEORGE

FIELD, R H

BOVE MOTORS

DICKENS, I L

-- ? 30

CUSHING,H L

DARL ING'S REST

DARL ING , FRED

MCCLEOD, F

$\begin{array}{rrr}-- & D & 30 \\ -- & D & 48 \\ 1949 & C & 6 \\ -- & C & 6\end{array}$

1952

TREMBLEY,P

BRAGAUL, ANTONE

HALL, M L

SANTOS, MANUEL

BRETTO,MAY

$1948 \mathrm{C}$

$1930 \mathrm{C}$

$\begin{array}{ll}1928 & C \\ 1928 & C\end{array}$

VIERRA, MANUEL 1930 C $1952 \quad C$ $1949 \mathrm{C}$

KANE, $B$ N

SMITH,E W

1935

$\begin{array}{lll}\text { ZAJCHOWSKI,S } & 1938 & \mathrm{C} \\ \text { OLIVERA, CLUDINO } & 1938 & \mathrm{C}\end{array}$

MELLO,MANUEL 1938 C

THOMPSON,H F

ROSA, JAMES

SEEKONK TOWN

ROSS,E GERTRUDE

SWANSON, $V R$

SWANSON, $V$
PRINCE, $L$

$x \quad 230 w 120$

$120 W$
80

$80 W \quad 25$

14

245

84
130
160

18
30
135
143
99

28

82

13

$22 w$

16
166

250
106

$247 w$

80

180

$110 w$

$19 w$

$\begin{array}{rl}75 & U \\ 280 & T\end{array}$

280
64

15
120
17

17
120

$120 w$

12
462
17
20

122
134

80
18
17

$\begin{array}{lll}- & D & 30 \\ = & D & 30\end{array}$

MUZZEY,RICHARD

BESSE, EVA

SEEKONK TOWN

GREEN,ELLIS
CLOVERLY,N

CONLON, JAMES
OLD GRIST MILL

OLD GRIST MIL

SEEKONK WD

SEEKONK WD

SEEKONK WO

SEEKONK WD

SEEKONK WD

SEEKONK WD

SEEKONK WD

SEEKONK WD

SEEKONK WD

SEEKONK WD
SEEKONK WD $\because$

18

18

18

$18 \mathrm{~W} 20$

14
350

350
210
54

$54 \mathrm{U}$
$230 \mathrm{~W}$

$52 \quad$

30

188
36
23

46
36

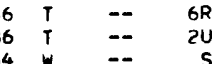

$\begin{array}{llll}34 & W & - & 5 \\ 40 & T & -- & T\end{array}$

1973
1973

1973

1973

1973
1973$$
\overline{-1}
$$

12

(FT) IURED I (GPM)I(FT)í(HR)

$$
\begin{aligned}
& \begin{array}{r}
-7 \\
18 \\
-12 \\
7 \\
14 \\
-- \\
27 \\
12 \\
6
\end{array} \\
& \begin{array}{cc}
-- & H \\
12-50 & H \\
-- & H \\
8-52 & 5 \\
8-52 & H
\end{array} \\
& \begin{array}{lr}
-- & 14 \\
-- & 5 \\
- & 16 \\
-- & 26 \\
& 16
\end{array} \\
& \begin{array}{cc}
8-52 & H \\
-- & H \\
-28 & H \\
10-49 & H \\
10-73 & H
\end{array} \\
& 11-49
\end{aligned}
$$$$
\begin{array}{rrrr}
15 & -- & - & -
\end{array}
$$$$
\begin{array}{rr}
8-52 & S \\
9-64 & - \\
2-46 & I \\
-35 & H \\
-35 & H
\end{array}
$$

$$
\begin{array}{r}
-51 \\
-46 \\
8-52
\end{array}
$$

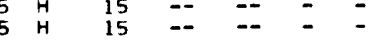$$
8-52 \quad H
$$$$
\begin{array}{rr}
8-52 & H \\
-49 & H
\end{array}
$$$$
8-52
$$$$
\begin{array}{ll}
6-48 & H \\
-- & H \\
-- & H \\
-28 & H \\
-- & S
\end{array}
$$$$
\begin{array}{cc}
8-52 & S \\
-- & C \\
-- & U \\
-52 & H
\end{array}
$$$$
8-5
$$$$
8-52
$$$$
8-52
$$$$
\begin{array}{rr}
-42 & 5 \\
8-52 & H \\
12-46 & H \\
8-52 & H \\
8-52 & H
\end{array}
$$

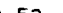$$
\begin{array}{ll}
-- & H \\
0-44 & \\
9-52 & \\
9-52 &
\end{array}
$$$$
9-52
$$$$
9-52 \quad H
$$$$
-28 \mathrm{H}
$$$$
9-52
$$$$
\begin{array}{ll}
9-52 & H \\
-- & H \\
-47 & H
\end{array}
$$$$
-39 \quad
$$$$
\therefore \quad-10
$$$$
-39
$$$$
1
$$$$
\begin{array}{r}
-32 \\
8-45
\end{array}
$$$$
\text { -- }
$$$$
\text { (3) }
$$$$
\begin{aligned}
& -- \\
& -- \\
& 18 \\
& --
\end{aligned}
$$

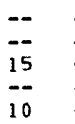$$
\begin{array}{r}
35 \\
-- \\
2 \\
20
\end{array}
$$$$
\begin{array}{r}
-- \\
--2 \\
5 \\
6
\end{array}
$$$$
=
$$$$
\begin{aligned}
& 12 \\
& -20 \\
& --
\end{aligned}
$$ 
LOCAL

WELL LATITUDE- ALTI - TUDE LONGITUDE TUDE (FT)
192415139 N0712001.1 i 93415130 N0712005.1 W 194 415125N0712001.1 W $195 \quad 415103 N 0712010.1$

W $199414803 N 0712018.1$ $200414806 N 0712025.1$ $203414847 N 0712001.1$
$209415223 N 0711937.1$ 214 415120N0712000.1

W $215 \quad 415113 N 0711954.1$ $2164 ! 5148 N 0711940.1$ $217415145 \mathrm{~N} 0711936.1$ W 220 415148N0711929.1 222415154 NO711940.1 $223415152 N 0711900.1$ $244415051 N 0712027.1$ 245415006 N0712010.1

W $246415006 N 0711958.1$ W 247 414858N0712020.1 248 415110NOT12001.1

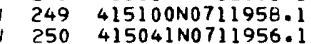

251 415202N0712003.1 W 255 414849N0712021.1 256 414821N0712021.1 W 258 414713NO711913.1

W $259414707 N 0711855.1$ W 264 415125N0712009.1 W 415125N0712009.? 266 415124N0712012.1 275414710 N0711753.1

$284 \quad 415037 \mathrm{~N} 0712030.1$ $313415123 N 0712009.1$
$1415042 N 0712005.1$ w

70
71
61
77
55
18
11
48
75
55
68
59
66
63
65
61
68
89
60
59
60
57
65
73
65
60
35
17
20
33
28
65
67
64
21
50
63
60

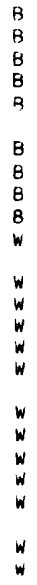

$\begin{array}{rlr}10 & 414339 N 0711131.1 & -9 \\ 1 & 414424 N 0711114.1 & -5 \\ 2 & 414243 N 0711032.1 & 6 \\ 3 & 414238 N 0711024.1 & -1 \\ 14 & 414234 N 0711016.1 & -17 \\ 15 & 414230 N 0711008.1 & -18 \\ 16 & 414307 N 0711100.1 & 72 \\ 17 & 414342 N 0711120.1 & 27 \\ 8 & 414333 N 0711112.1 & 27 \\ 1 & 414253 N 0711025.1 & 19 \\ & & \\ 6 & 414312 N 0711054.1 & 105 \\ 8 & 414311 N 0711046.1 & 100 \\ 9 & 414239 N 0711047.1 & 15 \\ 10 & 414239 N 0711053.1 & 15 \\ 12 & 414335 N 0711042.1 & 95 \\ 13 & 414335 N 0711046.1 & 85 \\ 20 & 414405 N 07111040.1 & 105 \\ 24 & 414429 N 0711018.1 & 158 \\ 26 & 414430 N 0711024.1 & 154 \\ 27 & 414425 N 0711028.1 & 155 \\ & & \\ 31 & 414405 N 0711020.1 & 154 \\ 33 & 414430 N 0711106.1 & 25 \\ 4 & 414341 N 0711127.1 & 12\end{array}$

$\begin{array}{llll}1 & 414619 N 0711700.1 & -9 & M D P W \\ 2 & 414401 N 0711256.1 & -14 & M D P W\end{array}$

$3414446 N 0711209.1 \quad-2$ MDPW

$514612 N 0711450$.

6414520 NO $111330.1 \quad 105$ $7414459 N 0711308$.

B 414349N0711133.1

9
10 414359 NOT111150.1

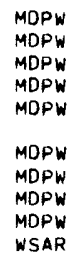

MDPW

$M D P W$

MDPW

MDPW

$M D P W$
MDPW

MDPW

MDPW

MOPW

MDPW

MDPW
SEEKONK WD SEEKONK WD SEEKONK WD SEEKONK WO
SEEKONK WD

SEEKONK WO SEEKONK WD SEEKONK WD SEEKONK WD

SEEKONK WD

LEDGEMONT C CL

LEDGEMONT C CL

LEDGEMONT $\mathrm{C} \mathrm{CL}$

LEDGEMONT $C \mathrm{CL}$

LEOGEMONT C CL

LEOGEMONT $\mathrm{C} \mathrm{Cl}$

SEEKONK WD
SEEKONK WD

SEEKONK WD

SEEKONK WD

SEEKONK WD

SEEKONK WD

SEEKONK WO

SEEKONK WD

SEEKONK WD

SEEKONK WD

SEEKONK WD

SEEKONK WD

SEEKONK WD

SEEKONK WD
US GEOL SURVEY

EAST PROVIDENCE

SEEKONK WD
SEEKONK TOWN

WSAR RADIO STA

MEDE IROS, ANTONE

AUSTIN, HERBEFT

KERSHAW, E

OLIVAL, E

BONADIE.G N

DUQUETTE , LEO

KUDLACIK.MA
KALILE,C

GONCALO, MANUEL

COREAU, ARTHUR

LEITE, J JOHNSONS 1965
HOWARD JO

1974 W

1971

1970
1970

$1970 \mathrm{~W}$

1970
1970
1952

$1952 W$

1952

1952
1952

1952

$1952 \mathrm{~W}$

1952
1052

1952
1952

$1952 W$

$1952 \mathrm{~W}$

$1958 \quad C$

1958 W

$1959 \mathrm{~B}$

$1959 \quad W$

1959 B
WELL

YEAR/ DETAM-IFIN-IDEPTHIUSE TEET WATER-

WATER

PUMPAGE (IN) I I (FT) I ROCK

SEEKONK --CONTINUED

$\begin{array}{rlllllllllllllll}1973 & W & 2 & S & 60 & T & -- & 3 S & -- & -- & U & 20 & -- & -- & D & - \\ 1973 & W & 2 & P & 90 & T & -- & 7 S & 27 & 7-73 & U & Z & -- & -- & D & - \\ 1974 & C & 8 & S & 69 & T & 69 & 3 R & 17 & 5-74 & U & 450 & 12 & 480 & D & M \\ 1973 & W & 2 & P & 15 & T & -- & T & -- & -- & U & -- & -- & -- & D & - \\ 1973 & W & 2 & P & 55 & T & -- & 2 U & -- & -- & U & -- & -- & -- & D & -\end{array}$

$\begin{array}{llll}1973 & W & 2 & S \\ 1973 & W & 2 & P \\ 1973 & W & 2 & P \\ 1974 & W & 2 & S \\ 1974 & W & 2 & S\end{array}$

$\begin{array}{llll}69 & T & -- & 3 R \\ 79 & T & -- & P U \\ 30 & T & -- & 6 G \\ 50 & T & -- & 4 R \\ 36 & T & -- & 4 R\end{array}$

2
18
2

$\begin{array}{llll}40 & T & -- & 4 R \\ 40 & W & -- & -- \\ 41 & T & -- & 3 R \\ 45 & T & -- & 3 R \\ 36 & T & -- & 3 R\end{array}$

$\begin{array}{llll}47 & T & -- & 2 R \\ 31 & T & -- & 2 R\end{array}$

$\begin{array}{llll}45 & T & -- & 2 R \\ 72 & T & -- & 7 R \\ 5 & T & -- & P U\end{array}$

$\begin{array}{llll}56 & T & -- & -\end{array}$

$\begin{array}{ccc}11 & 8-73 & U \\ -- & -- & U \\ -- & -- & U \\ -- & -- & U \\ 0 & 2-74 & U\end{array}$

$\begin{array}{rrrr}35 & -- & =- & 0 \\ Z & =- & = & 0 \\ 25 & =- & = & 0 \\ 15 & = & =- & 0\end{array}$

$4-74$
$11-71$

$$
\begin{array}{lr}
1 & 11-7 \\
8 & 9-7 \\
6 & 9-70
\end{array}
$$

\begin{tabular}{rrrrrr}
-70 & $U$ & 300 & 26 & 48 & $D$ \\
\hline-70 & & 60 & 4 & 3 & $D$
\end{tabular}

$\begin{array}{rrrrrr}9-70 & U & 65 & -- & 3 & 0 \\ 9-70 & U & -- & -- & -- & 0\end{array}$

$9-70$ $9-70$ U $9-70$
$10-52$

20

$11-52$ u 13

$\begin{array}{llll}53 & T & -- & 65 \\ 45 & T & -- & P U \\ 41 & T & -- & 35 \\ 67 & T & -- & 2 U \\ 49 & T & -- & P U\end{array}$

13 -- $=$

\section{$\begin{array}{ll}2 & p \\ 2 & p \\ 2 & S \\ 2 & P \\ 2 & P\end{array}$}

$\begin{array}{ccc}-- & -- & U \\ -- & -- & U \\ 4 & 11-52 & U \\ -- & -- & U \\ -- & -- & U\end{array}$

$--$

$\because \because$

$\begin{array}{llll}31 & T & -- & 2 R \\ 32 & T & -- & P U \\ 56 & T & -- & 9 P \\ 44 & T & -- & T\end{array}$

59

-- $Q P$

60

66 W -

14

$\begin{array}{llll}81 & T & -- & U \\ 83 & W & -- & 4 R \\ 45 & T & -- & --\end{array}$

$\begin{array}{rr}2 & P \\ 24 & G\end{array}$

SOMERSE T

$\begin{array}{rrrrrrrrrrrr}21 & T & -- & -- & -- & -- & U & -- & -- & -- & 0 & - \\ 19 & T & -- & -- & -- & -- & U & -- & -- & -- & 0 & - \\ 62 & 1 & 57 & -- & -- & -- & U & -- & -- & -- & 0 & - \\ 68 & 1 & 63 & -- & -- & -- & U & -- & -- & -- & 0 & - \\ 120 & T & 115 & -- & -- & -- & U & -- & -- & -- & 0 & -\end{array}$

$\begin{array}{rrrrr}140 & T & 134 & =- & - \\ 25 & T & -- & = & -- \\ 25 & T & 10 & =- & \\ 30 & T & 19 & =- & \\ 300 & W & 30 & --\end{array}$

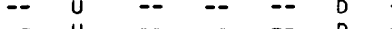

$\begin{array}{lllll}26 & U & -- & -- & 12 \\ 28 & W & -- & -- & 23 \\ 16 & W & -- & -- & 13 \\ 103 & U & 60 & -- & -\end{array}$

$9-59 \quad U$

--

$-47$

$9-52 \quad U$

$\begin{array}{ll}9-52 & H \\ 9-52 & H \\ --- & U\end{array}$

$9-52 \quad H$

110

102

65
70
22

$\begin{array}{ll}-5 & H \\ -52 & H\end{array}$

$10-52 \quad H$

$10-52 \quad H$

$\begin{array}{rrrrrr}16 & U & 12 & -- & 9 & 10-52 \\ 23 & W & -- & -- & 15 & 10-52\end{array}$

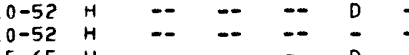

SWANSEA

$\begin{array}{cccc}1938 & - & -- & 0 \\ 1925 & - & -- & 0 \\ -- & - & -- & 0 \\ 1958 & 8 & 2 & x \\ 1958 & 8 & 2 & x \\ 1958 & B & 2 & x \\ 1958 & 8 & 2 & x \\ 1959 & W & 2 & 0 \\ 1959 & 8 & 2 & x \\ 1959 & W & 2 & 0\end{array}$

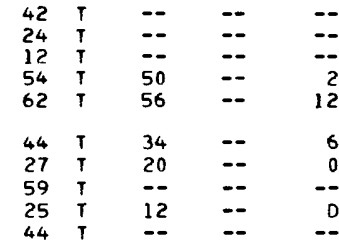


WATER

PUMPAGE

METHOD DIAM-IFIN-IDEPTHIUSE

FEET WATER-

$$
\text { SWANSEA --CONTINUED }
$$

MEAS-1

YIELOI DD ITIME LDG QW

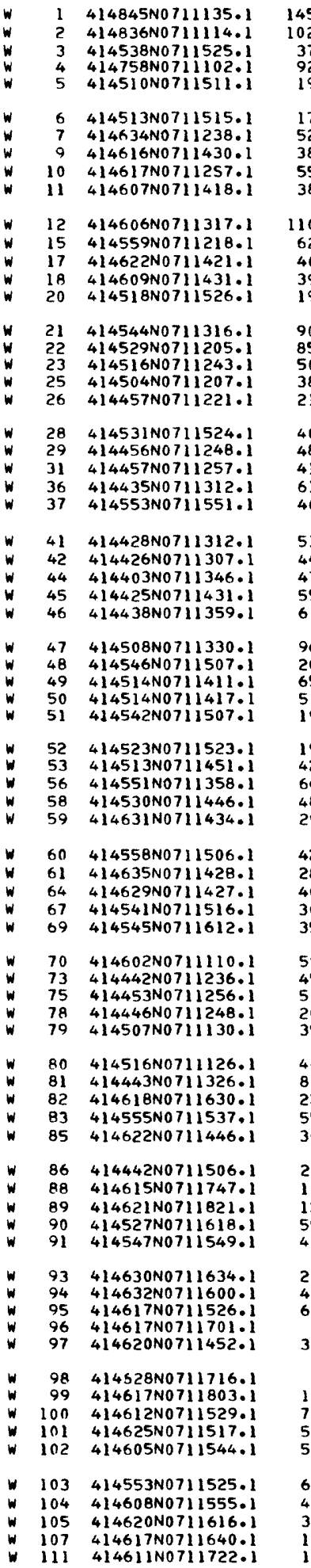

REESE , ANTHONY SYLVIA,R SWANSEA WO
CHACE, MERRILL CHACE, MERRI SWANSEA WD

SWANSEA WO
BAKER,MYRON BAKER, MYRON BRISTOL CO WAT BRISTOL CO WAT $\begin{array}{rrr}-- & D & 30 \\ -- & C & 6 \\ 1971 & W & 2 \\ -- & 0 & 30 \\ 1950 & C & 24\end{array}$ HALE, WILL I AM ESPANIOLA BRISTOL CO WAT BRISTOL CD WAT 5WANSEA WO

SWANSEA BOTTLE

SUPRENARO WOODS DAIRY BORGE

\section{SWANSEA WO}

PRINCETON DINER

GULF ICE CREAM

SWANALA MANUE

RECH, RAYMOND

WALKER, IAN

PELLETIER, N

HOLT

CORREIA,F

\section{MAOIERAS}

SWANSEA WO

DE ISS, CHARLES

SWANSEA WO

$1950 \mathrm{C}$

1945

$\overline{--} \quad$ W

24
30
--

$\begin{array}{lll}-- & C & \end{array}$

$\begin{array}{rrr}-- & C & 6 \\ - & 0 & 30 \\ 945 & W & --\end{array}$

945

1945
1957

$\begin{array}{ll}-- & c \\ -- & c \\ -- & c \\ -- & 0\end{array}$

SWANSEA WD

CHACE, GEORGE

BRISTOL CO WAT

MELLOW,F
SWANSEA WO

VINNI CUM.LYOIA

SWANSEA WO

TRIMBLE, T

LUTHER, $C R$

SWANSEA WD

SOROKA S $S$

SWANSEA WO

SWANSEA WD

STRACHMAN FARM

5WANSEA TOWN

MARTIN HDUSE

SWANSEA WO

YOST, C
YOOO,

WOOD, E J

ARROWHEAD FARM

JOHNSON, C E

1971

1932 C

1971 w

$1 \overline{932}$

$1948 \mathrm{C}$

$=\quad C$

$1972 \quad$ W

‥ $\quad c$

--
1957

19570

1974

$1936 \mathrm{C}$

1971
1971

--

1971

$1030 \mathrm{C}$

1957
1957

1957

$\begin{array}{cc}-- & C \\ -- & C \\ 1920 & C\end{array}$

1957 w

BARNEY,A S

DEMARAIS HENRY

BELL.A H

$1950 \mathrm{C}$

1948

1952

BRA ILEY, ALFRED

TERRY, E J

DOYLE,E P
FIGURIEO, TONY

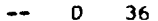

DEAN, CARROLL

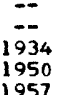

1957

FIOLA, V

CEOAR REST TOUR

BAKER, C B

BAKNEY, JESSE
MARTIN,HDMER

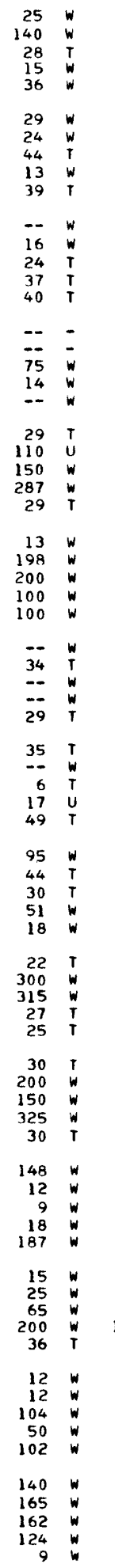

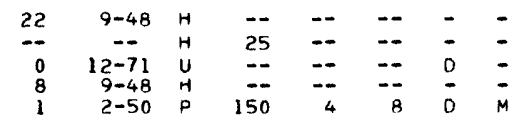

$\begin{array}{cccccccc}0 & 4-50 & P & 150 & 9 & 72 & 0 & M \\ 10 & 9-48 & H & -- & -- & -- & - & - \\ -- & -- & U & -- & - & - & - & - \\ 9 & 9-48 & S & -- & - & - & - & - \\ -- & -- & U & -- & -- & -- & - & -\end{array}$

$\begin{array}{ccccc}9-48 & H & -- & -- & - \\ -- & U & -- & -- & --\end{array}$

$\begin{array}{lllll}-- & U & -- & -- & -- \\ -- & U & -- & = & --\end{array}$ 
LOCAL

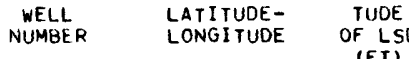
(FT)

\begin{tabular}{|c|c|c|c|}
\hline & $\begin{array}{l}115 \\
119 \\
119 \\
122 \\
124\end{array}$ & $\begin{array}{l}414612 N 0711603.1 \\
414428 N 0711216.1 \\
414358 N 0711310.1 \\
414303 N 0711336.1 \\
414358 N 0711416.1\end{array}$ & $\begin{array}{l}28 \\
42 \\
25 \\
15 \\
35\end{array}$ \\
\hline $\mathbf{w}$ & $\begin{array}{l}125 \\
126 \\
127 \\
128 \\
129\end{array}$ & $\begin{array}{l}414415 N 0711451.1 \\
414709 N 0711052.1 \\
414433 N 0711410.1 \\
414411 N 0711303.1 \\
414434 N 0711341.1\end{array}$ & $\begin{array}{l}55 \\
78 \\
74 \\
45 \\
49\end{array}$ \\
\hline$W$ & $\begin{array}{l}130 \\
132 \\
133 \\
134 \\
136\end{array}$ & $\begin{array}{l}414238 N 0711243.1 \\
414251 N 0711247.1 \\
414247 N 0711239.1 \\
414237 N 0711224.1 \\
414300 N 0711217.1\end{array}$ & $\begin{array}{r}29 \\
9 \\
40 \\
26 \\
47\end{array}$ \\
\hline$w$ & $\begin{array}{l}137 \\
140 \\
141 \\
142 \\
143\end{array}$ & $\begin{array}{l}414256 N 0711228.1 \\
414313 N 0711212.1 \\
414715 N 0711042.1 \\
414301 N 0711227.1 \\
414242 N 0711210.1\end{array}$ & $\begin{array}{r}40 \\
55 \\
95 \\
35 \\
6\end{array}$ \\
\hline$w$ & $\begin{array}{l}144 \\
145 \\
146 \\
149 \\
151\end{array}$ & $\begin{array}{l}414414 N 0711141.1 \\
414420 N 0711142.1 \\
414432 N 0711138.1 \\
414458 N 0711140.1 \\
414449 N 0711120.1\end{array}$ & $\begin{array}{l}33 \\
31 \\
30 \\
40 \\
30\end{array}$ \\
\hline $\begin{array}{l}w \\
w \\
w\end{array}$ & $\begin{array}{l}153 \\
154 \\
161 \\
162 \\
165\end{array}$ & $\begin{array}{l}414453 N 0711057.1 \\
414455 N 0711146.1 \\
414639 N 07111022.1 \\
414432 N 0711324.1 \\
414339 N 0711234.1\end{array}$ & $\begin{array}{r}83 \\
38 \\
140 \\
75 \\
12\end{array}$ \\
\hline $\begin{array}{l}w \\
w\end{array}$ & $\begin{array}{l}166 \\
174 \\
176 \\
179 \\
180\end{array}$ & $\begin{array}{l}414435 N 0711015.1 \\
414431 N 0711005.1 \\
414349 N 0711153.1 \\
414332 N 0711212.1 \\
414753 N 0711032.1\end{array}$ & $\begin{array}{r}162 \\
185 \\
65 \\
70 \\
98\end{array}$ \\
\hline$W$ & $\begin{array}{l}182 \\
183 \\
186 \\
187 \\
188\end{array}$ & $\begin{array}{l}414813 N 0711037.1 \\
414254 N 0711208.1 \\
414814 N 0711032.1 \\
414407 N 0711335.1 \\
414403 N 0711322.1\end{array}$ & $\begin{array}{l}89 \\
25 \\
97 \\
53 \\
55\end{array}$ \\
\hline 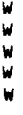 & $\begin{array}{l}190 \\
195 \\
196 \\
199 \\
200\end{array}$ & $\begin{array}{l}414806 N 0711046.1 \\
414 R 49 N 0711057.1 \\
414851 N 0711052.1 \\
414849 N 0711048.1 \\
414847 N 0711043.1\end{array}$ & $\begin{array}{l}89 \\
87 \\
86 \\
85 \\
86\end{array}$ \\
\hline$w$ & $\begin{array}{l}201 \\
206 \\
207 \\
213 \\
217\end{array}$ & $\begin{array}{l}414850 N 07111040.1 \\
414654 N 0711126.1 \\
414301 N 0711208.1 \\
414642 N 0711130.1 \\
414650 N 0711132.1\end{array}$ & $\begin{array}{l}86 \\
77 \\
40 \\
72 \\
75\end{array}$ \\
\hline$w$ & $\begin{array}{l}218 \\
220 \\
221 \\
222 \\
223\end{array}$ & $\begin{array}{l}414706 N 0711035.1 \\
414720 N 0711043.1 \\
414728 N 0711045.1 \\
414735 N 0711046.1 \\
414823 N 0711102.1\end{array}$ & $\begin{array}{l}99 \\
98 \\
99 \\
98 \\
89\end{array}$ \\
\hline$W$ & $\begin{array}{l}224 \\
225 \\
229 \\
230 \\
232\end{array}$ & $\begin{array}{l}414829 N 0711100.1 \\
414646 \mathrm{~N} 0711126.1 \\
414726 \mathrm{~N} 0711031.1 \\
414800 \mathrm{~N} 0711118.1 \\
414820 \mathrm{~N} 0711123.1\end{array}$ & $\begin{array}{l}89 \\
76 \\
98 \\
85 \\
92\end{array}$ \\
\hline $\begin{array}{l}w \\
w \\
w\end{array}$ & $\begin{array}{l}233 \\
235 \\
237 \\
238 \\
239\end{array}$ & $\begin{array}{l}414454 \mathrm{~N} 0711208.1 \\
414422 \mathrm{~N} 0711440.1 \\
414445 \mathrm{~N} 0711202.1 \\
414449 \mathrm{~N} 0711201.1 \\
414631 \mathrm{~N} 0711437.1\end{array}$ & $\begin{array}{l}19 \\
36 \\
19 \\
20 \\
35\end{array}$ \\
\hline$w$ & $\begin{array}{l}240 \\
241 \\
242 \\
244 \\
246\end{array}$ & $\begin{array}{l}414440 N 0711146.1 \\
414525 N 0711508.1 \\
414446 N 0711237.1 \\
414438 N 0711253.1 \\
414735 N 0711023.1\end{array}$ & $\begin{array}{r}29 \\
40 \\
35 \\
18 \\
122\end{array}$ \\
\hline 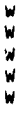 & $\begin{array}{l}249 \\
250 \\
251 \\
252 \\
259\end{array}$ & $\begin{array}{l}414601 N 0711230.1 \\
414604 N 0711243.1 \\
414607 N 0711246.1 \\
414609 N 0711245.1 \\
414559 N 0711244.1\end{array}$ & $\begin{array}{l}40 \\
37 \\
38 \\
38 \\
28\end{array}$ \\
\hline
\end{tabular}
YEAR, WELL DRILLED ETER IISH I BED- MATERIAL
ROCK (IN) I

SWANSEA --CONTINUED

\begin{tabular}{|c|c|}
\hline $\begin{array}{l}\text { TOPSY'S } \\
\text { SEARS, ROY H } \\
\text { SOUVIER, A } \\
\text { OEAUDRY, ARTHUR } \\
\text { WILLIAMS, C B }\end{array}$ & $\begin{array}{c}1933 \\
1931 \\
1951 \\
-- \\
-\end{array}$ \\
\hline $\begin{array}{l}\text { SEYMOUR, W } \\
\text { SWANSEA WD } \\
\text { RATUS }\end{array}$ & $\begin{array}{l}1947 \\
1969 \\
1941\end{array}$ \\
\hline $\begin{array}{l}\text { BUFF INGION,A C } \\
\text { REILLY,G J }\end{array}$ & 1949 \\
\hline $\begin{array}{l}\text { ANTHONY,C C } \\
\text { CEDAR COVE C CL } \\
\text { SMITH,C C } \\
\text { THOMPSON,R } \\
\text { BERUBE, ARTHUR }\end{array}$ & $\begin{array}{l}1923 \\
1949 \\
1951 \\
1930 \\
--\end{array}$ \\
\hline $\begin{array}{l}\text { NORMAN, JOHN } \\
\text { GARONER, F L } \\
\text { SWANSEA WD } \\
\text { BERUBE, A JR } \\
\text { ROUNSEVILLE, C C }\end{array}$ & $\begin{array}{l}1931 \\
1940 \\
1969 \\
-- \\
1908\end{array}$ \\
\hline
\end{tabular}

CLVELTOATE IUSE
IMEAS-I (FT) IURED

PUMPAGE
SWANSEA WD

SWANSEA WO

SWANSEA WD

SWANSEA WD

5WANSEA WO

SWANSEA WO

5WANSEA WO

SWANSEA WO

SWANSEA WO

SWANSEA WD

SWANSEA WD

FERRY, JOHN

SWANSEA WO

SWANSEA WD

SWANSEA WD

SWANSEA WD
$1969 W$

$1969 \mathrm{R}$

1969

1969

$1948 W$

1969

1969

1948
1973

1948 W

1964
1948

1948

1965

$1948 w$

1948
1949

1949

\begin{tabular}{|c|c|c|c|c|c|c|c|c|}
\hline \multirow{4}{*}{$\begin{array}{l}\text { TOPSY'S } \\
\text { SEARS,ROY H } \\
\text { BOUVIER, A } \\
\text { BEAUDRY,ARTHUR } \\
\text { WILLI IAMS, C B }\end{array}$} & 1933 & c & 6 & $x$ & 184 & $w$ & 80 & -- \\
\hline & 1931 & C & 6 & $x$ & 310 & $w$ & -- & - \\
\hline & 1951 & c & 6 & $x$ & 102 & 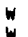 & 32 & -- \\
\hline & $\because$ & $c$ & 6 & $\hat{x}$ & 113 & 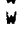 & 15 & - \\
\hline SEYMOUR,W & 1947 & c & 6 & $x$ & 200 & $\mathbf{W}$ & 24 & -- \\
\hline SWANSEA WD & 1969 & $w$ & 2 & $P$ & 31 & 1 & 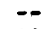 & PU \\
\hline FRATUS & 1941 & C & 6 & $x$ & 123 & $w$ & 40 & -- \\
\hline BUFF INGION, A C & -- & c & 6 & $x$ & 68 & $w$ & 20 & - \\
\hline REILLY,G J & 1949 & c & 6 & $x$ & 80 & $w$ & 18 & -- \\
\hline ANTHONY,C C & 1923 & c & 6 & $x$ & 200 & $u$ & 130 & -- \\
\hline CEOAR COVE C CL & 1949 & c & 6 & $x$ & 200 & $u$ & 120 & -- \\
\hline SMITH.C C & 1951 & c & 6 & $x$ & 215 & $w$ & 50 & -- \\
\hline $\begin{array}{l}\text { THOMPSON, R } \\
\text { BERUBE, ARTHUIR }\end{array}$ & 1930 & $\begin{array}{l}\mathrm{c} \\
\mathrm{c}\end{array}$ & $\begin{array}{l}6 \\
6\end{array}$ & $\begin{array}{l}x \\
x\end{array}$ & $\begin{array}{l}150 \\
450\end{array}$ & $u$ & 11 & $=$ \\
\hline NORMAN. JOHN & 1931 & $c$ & 6 & $x$ & 90 & $u$ & -- & -- \\
\hline GARONER, F L & 1940 & c & 6 & $\hat{x}$ & 142 & u & 30 & -- \\
\hline SWANSEA WD & 1969 & $w$ & 2 & $P$ & 44 & $T$ & $\bar{a}$ & PU \\
\hline BERUBE , A JR & -- & c & 6 & $x$ & 350 & $w$ & 25 & -- \\
\hline ROUNSEVILLE, C C & 1908 & c & 6 & $x$ & 50 & $u$ & - & -- \\
\hline LAFLAMME BROS & -- & D & 48 & $w$ & 24 & $u$ & -- & - \\
\hline BLISS,GEORGE & 1928 & $\mathrm{c}$ & 6 & $x$ & 67 & $u$ & 30 & -- \\
\hline & 1931 & c & 6 & $x$ & 62 & $w$ & 30 & $-\infty$ \\
\hline SWANSEA TOWN & -- & c & 6 & $x$ & 37 & $u$ & 21 & -- \\
\hline STEVENS HOME & 1937 & c & 6 & $x$ & 408 & $u$ & 6 & -- \\
\hline REST HOUSE & 1917 & c & 6 & $x$ & 165 & $u$ & 12 & -- \\
\hline SWANSEA TOWN & 1908 & c & 6 & $x_{0}^{x}$ & 55 & $w$ & 10 & -- \\
\hline $\begin{array}{l}\text { SWANSEA WD } \\
\text { SWANSEA TOWN }\end{array}$ & 1969 & $w$ & 2 & $P^{p}$ & 16 & $\mathrm{~T}$ & $\ddot{z}$ & -- \\
\hline $\begin{array}{l}\text { SWANSEA TOWN } \\
\text { ST MICHAELS SCH }\end{array}$ & 1950 & $c_{c}^{c}$ & 6 & $x$ & 280 & u & 100 & $=$ \\
\hline WYNNYK, GEORGE & 1929 & $c$ & 6 & $x$ & 70 & $w$ & -- & -- \\
\hline LEWIS, F & $\ldots$ & D & 30 & $\omega$ & 26 & $\ddot{u}$ & -- & - \\
\hline RICE, CHARLES & 1931 & c & 6 & $x$ & 72 & $w$ & -- & -- \\
\hline SULL IVAN,P & 1932 & c & 6 & $x$ & 102 & $u$ & -- & -- \\
\hline SWANSEA WD & 1969 & $w$ & 2 & $P$ & 22 & $\mathbf{T}$ & -- & PU \\
\hline SWANSEA WD & 1969 & $w$ & 2 & $P$ & 28 & $T$ & -- & PU \\
\hline KINGSLEY,F H & 1913 & c & 6 & $x$ & 194 & $w$ & $\cdots$ & -- \\
\hline SWANSEA WD & 1969 & w & 2 & $p$ & 22 & $\boldsymbol{T}$ & -- & PU \\
\hline HAYDEN,D & 1937 & c & 6 & $x$ & 70 & $\omega$ & 5 & -- \\
\hline LESHINSKY,A & 1929 & c & 6 & $x$ & 94 & $w$ & -- & -- \\
\hline SWANSEA WD & 1969 & $w$ & 2 & $\mathrm{p}$ & 32 & $\mathbf{T}$ & - & $T$ \\
\hline SWANSEA WD & 1969 & $\omega$ & 2 & $p$ & 13 & $\mathrm{~T}$ & -- & -- \\
\hline SWANSEA WD & 1969 & $w$ & 2 & $\mathbf{s}$ & 28 & $T$ & -- & $2 R$ \\
\hline SWANSEA WD & 1969 & 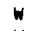 & 2 & 0 & 30 & $T$ & - & $3 R$ \\
\hline SWANSEA WD & 1969 & w & 2 & $\mathrm{P}$ & 23 & $\mathrm{~T}$ & -- & $3 R$ \\
\hline SWANSEA WD & 1969 & $w$ & 2 & $\mathrm{P}$ & 30 & $T$ & - & $3 R$ \\
\hline SWANSEA WD & 1969 & 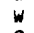 & 2 & $P$ & 32 & $T$ & -- & $2 R$ \\
\hline CULLEN, JOHN R & 1920 & $c$ & 6 & $x$ & 124 & $u$ & -- & - \\
\hline SWANSEA WO & 1969 & w & 4 & $\mathrm{~s}$ & 27 & $w$ & -- & $3 R$ \\
\hline SWANSEA WD & 1969 & $w$ & 2 & $\mathrm{P}$ & 19 & $\mathrm{~T}$ & -- & - \\
\hline SWANSEA WD & 1969 & $w$ & 2 & $\mathrm{~s}$ & 28 & $T$ & -- & $2 R$ \\
\hline SWANSEA WO & 1969 & $\omega$ & 2 & p & 28 & $t$ & -- & $6 R$ \\
\hline SWANSEA WD & 1969 & w & 2 & $P$ & 17 & $T$ & 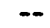 & - \\
\hline SWANSEA WD & 1969 & $w$ & 2 & $P$ & 22 & $\mathrm{~T}$ & -- & $6 R$ \\
\hline SWANSEA & 1769 & & 2 & $P$ & 26 & $\mathbf{T}$ & -- & $3 R$ \\
\hline
\end{tabular}

\begin{tabular}{|c|c|c|c|c|c|}
\hline 6 & $x$ & 184 & $w$ & 80 & -- \\
\hline 6 & $x$ & 310 & 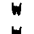 & $\overline{32}$ & $=-$ \\
\hline & $\begin{array}{l}x \\
x\end{array}$ & 97 & $w$ & $\begin{array}{l}32 \\
40\end{array}$ & $=$ \\
\hline & & & $w$ & & -- \\
\hline & $x$ & 200 & $w$ & 24 & -- \\
\hline & $P$ & 31 & $T$ & -- & PU \\
\hline & $x$ & 123 & $w$ & 40 & -- \\
\hline & $x$ & 68 & $w$ & 20 & -- \\
\hline 6 & & & 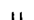 & 30 & -- \\
\hline 0 & $\hat{x}$ & 200 & u & 120 & -- \\
\hline 6 & $x$ & 215 & 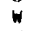 & 50 & -- \\
\hline 6 & $x$ & 150 & $u$ & 11 & -- \\
\hline 6 & $x$ & 450 & u & -- & -- \\
\hline 6 & $x$ & 90 & $u$ & -- & -- \\
\hline 6 & $x$ & 142 & $u$ & 30 & -- \\
\hline 2 & $P$ & 44 & $T$ & $\overline{-}$ & PU \\
\hline $\begin{array}{l}6 \\
6\end{array}$ & $\begin{array}{l}x \\
x\end{array}$ & $\begin{array}{r}350 \\
50\end{array}$ & $w$ & 25 & $=-$ \\
\hline 48 & $w$ & 24 & $u$ & -- & -- \\
\hline 6 & $x$ & 67 & $u$ & 30 & -- \\
\hline 6 & $x$ & 62 & $w$ & 30 & -- \\
\hline 6 & $x$ & 37 & u & 21 & -- \\
\hline 6 & $x$ & 408 & 0 & 6 & -- \\
\hline 6 & $x$ & 165 & $u$ & 12 & -- \\
\hline${ }^{6}$ & ${ }_{p}^{x}$ & $\begin{array}{l}55 \\
16\end{array}$ & $w$ & 10 & $=-$ \\
\hline 6 & $x$ & 280 & $w$ & 15 & -- \\
\hline 6 & $x$ & 125 & $u$ & 100 & -- \\
\hline 6 & $x$ & 70 & $w$ & -- & -- \\
\hline 30 & $w$ & 26 & u & - & -- \\
\hline 6 & $x$ & 102 & u & $=$ & $=$ \\
\hline 2 & $\hat{\mathrm{P}}$ & 22 & $T$ & $=$ & PU \\
\hline 2 & $P$ & 28 & $T$ & -- & PU \\
\hline 6 & $\begin{array}{l}x \\
0\end{array}$ & 194 & $w$ & -- & $\ddot{-}$ \\
\hline 2 & $P$ & 22 & $\stackrel{T}{T}$ & 5 & -- \\
\hline 6 & $x$ & 94 & $w$ & -- & -- \\
\hline 2 & $P$ & 32 & $\boldsymbol{T}$ & -- & $T$ \\
\hline & $p$ & 13 & $I$ & -- & $=$ \\
\hline 2 & 0 & $\begin{array}{l}28 \\
30\end{array}$ & T & $\ldots$ & $\begin{array}{l}2 R \\
3 R\end{array}$ \\
\hline 2 & $P$ & 23 & $T$ & - & $3 R$ \\
\hline 2 & $P$ & 30 & $T$ & -- & $3 R$ \\
\hline ? & $x$ & 32 & $T$ & - & $2 R$ \\
\hline . & $\hat{\mathbf{s}}$ & 124 & U & -- & $\overrightarrow{3 R}$ \\
\hline 2 & $\vec{P}$ & 19 & $\mathrm{~T}$ & 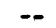 & -. \\
\hline 2 & $S$ & 28 & $\mathbf{T}$ & -- & $2 R$ \\
\hline 2 & $P$ & 28 & $T$ & -- & $6 R$ \\
\hline 2 & $p$ & $\begin{array}{l}11 \\
22\end{array}$ & T & $\therefore$ & $6 R$ \\
\hline & $p$ & 26 & T & -. & $3 R$ \\
\hline
\end{tabular}

\begin{tabular}{|c|c|c|c|c|c|}
\hline 6 & $x$ & 184 & $w$ & 80 & -- \\
\hline 6 & $x$ & 310 & 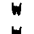 & $\overline{32}$ & $=-$ \\
\hline & $\begin{array}{l}x \\
x\end{array}$ & 97 & $w$ & $\begin{array}{l}32 \\
40\end{array}$ & $=$ \\
\hline & & & $w$ & & -- \\
\hline & $x$ & 200 & $w$ & 24 & -- \\
\hline & $P$ & 31 & $T$ & -- & PU \\
\hline & $x$ & 123 & $w$ & 40 & -- \\
\hline & $x$ & 68 & $w$ & 20 & -- \\
\hline 6 & & & 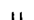 & 30 & -- \\
\hline 0 & $\hat{x}$ & 200 & u & 120 & -- \\
\hline 6 & $x$ & 215 & 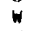 & 50 & -- \\
\hline 6 & $x$ & 150 & $u$ & 11 & -- \\
\hline 6 & $x$ & 450 & u & -- & -- \\
\hline 6 & $x$ & 90 & $u$ & -- & -- \\
\hline 6 & $x$ & 142 & $u$ & 30 & -- \\
\hline 2 & $P$ & 44 & $T$ & $\overline{-}$ & PU \\
\hline $\begin{array}{l}6 \\
6\end{array}$ & $\begin{array}{l}x \\
x\end{array}$ & $\begin{array}{r}350 \\
50\end{array}$ & $w$ & 25 & $=-$ \\
\hline 48 & $w$ & 24 & $u$ & -- & -- \\
\hline 6 & $x$ & 67 & $u$ & 30 & -- \\
\hline 6 & $x$ & 62 & $w$ & 30 & -- \\
\hline 6 & $x$ & 37 & u & 21 & -- \\
\hline 6 & $x$ & 408 & 0 & 6 & -- \\
\hline 6 & $x$ & 165 & $u$ & 12 & -- \\
\hline${ }^{6}$ & ${ }_{p}^{x}$ & $\begin{array}{l}55 \\
16\end{array}$ & $w$ & 10 & $=-$ \\
\hline 6 & $x$ & 280 & $w$ & 15 & -- \\
\hline 6 & $x$ & 125 & $u$ & 100 & -- \\
\hline 6 & $x$ & 70 & $w$ & -- & -- \\
\hline 30 & $w$ & 26 & u & - & -- \\
\hline 6 & $x$ & 102 & u & $=$ & $=$ \\
\hline 2 & $\hat{\mathrm{P}}$ & 22 & $T$ & $=$ & PU \\
\hline 2 & $P$ & 28 & $T$ & -- & PU \\
\hline 6 & $\begin{array}{l}x \\
0\end{array}$ & 194 & $w$ & -- & $\ddot{-}$ \\
\hline 2 & $P$ & 22 & $\stackrel{T}{T}$ & 5 & -- \\
\hline 6 & $x$ & 94 & $w$ & -- & -- \\
\hline 2 & $P$ & 32 & $\boldsymbol{T}$ & -- & $T$ \\
\hline & $p$ & 13 & $I$ & -- & $=$ \\
\hline 2 & 0 & $\begin{array}{l}28 \\
30\end{array}$ & T & $\ldots$ & $\begin{array}{l}2 R \\
3 R\end{array}$ \\
\hline 2 & $P$ & 23 & $T$ & - & $3 R$ \\
\hline 2 & $P$ & 30 & $T$ & -- & $3 R$ \\
\hline ? & $x$ & 32 & $T$ & - & $2 R$ \\
\hline . & $\hat{\mathbf{s}}$ & 124 & U & -- & $\overrightarrow{3 R}$ \\
\hline 2 & $\vec{P}$ & 19 & $\mathrm{~T}$ & 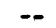 & -. \\
\hline 2 & $S$ & 28 & $\mathbf{T}$ & -- & $2 R$ \\
\hline 2 & $P$ & 28 & $T$ & -- & $6 R$ \\
\hline 2 & $p$ & $\begin{array}{l}11 \\
22\end{array}$ & T & $\therefore$ & $6 R$ \\
\hline & $p$ & 26 & T & -. & $3 R$ \\
\hline
\end{tabular}

$\begin{array}{llll}23 & T & -- & 2 R \\ 29 & T & -- & 2 R \\ 18 & T & - & - \\ 18 & T & - & 2 R\end{array}$

$\begin{array}{llll}27 & -= & P U\end{array}$

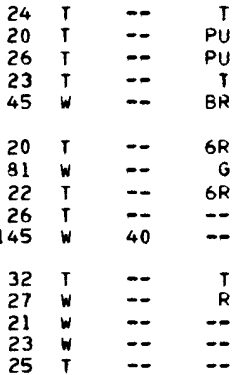

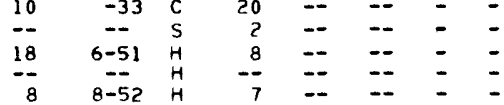

$\begin{array}{cccccccc}-- & -- & H & 24 & -- & - & - & - \\ 2 & 8-69 & U & -- & -- & - & 0 & =\end{array}$

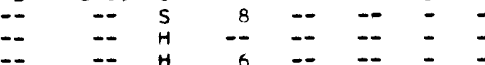

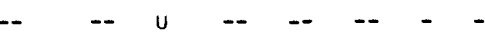

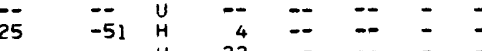

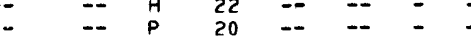

$\begin{array}{llllllll}-- & - & P & 50 & - & - & - & - \\ 20 & 9-52 & P & 25 & -- & -- & D & -\end{array}$

$\begin{array}{cccccccc}3 & 8-69 & U & -- & -- & -- & D & - \\ -- & -- & H & 8 & -- & -- & - & - \\ -- & H & H & 3 & -- & -- & - & -\end{array}$

$\begin{array}{cccccccc}13 & 9-52 & U & -- & -- & -- & - & - \\ -- & -- & H & 1 & -- & -- & - & - \\ 20 & -52 & H & 4 & -- & -- & - & -\end{array}$

20

$9-52$ U $\begin{array}{cccccc}-- & - & - & -- & 0 & -\end{array}$

$\begin{array}{lllllll}- & u & - & - & - & 0 & 0\end{array}$

$=\begin{array}{llllll}H & H & 15 & = & = & = \\ \because & H & - & = & = & =\end{array}$

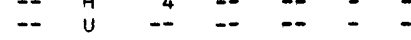

$\begin{array}{cccccc}-- & H & 15 & =- & = & - \\ 10-52 & H & -- & -- & = & =\end{array}$

$\begin{array}{llllll}-- & H & 6 & = & = & = \\ = & U & 2 & = & = & = \\ -- & U & -- & = & = & =\end{array}$

$\begin{array}{lllllll}9-69 & 4 & -- & -- & -- & 0 & -\end{array}$

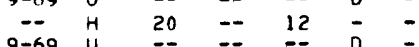

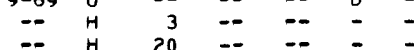

$\begin{array}{lllllll}9-69 & \cup & -- & -- & -- & D & -\end{array}$

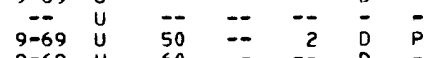

$\begin{array}{lllllll}9-69 & u & 60 & -- & -- & 0 & P \\ 9-69 & \cup & 60 & -- & -- & 0 & P\end{array}$

$\begin{array}{lllllll}9-69 & \cup & 65 & - & 2 & 0 & P\end{array}$

$\begin{array}{rrrrrrrr}1 & 10-69 & U & 60 & = & 4 & 0 & P \\ -1 & -- & U & -- & = & -\overline{0} & - & - \\ 1 & 10-69 & P & 137 & -- & 120 & 0 & M\end{array}$

$2 \quad 10-69$ U $20 \quad--\quad 8 \quad 0$

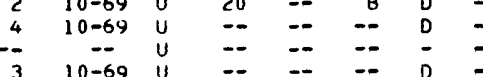

$\begin{array}{ccccccc}10-69 & u & -- & -- & -- & 0 & - \\ 10-69 & u & 65 & -- & 2 & 0 & \bar{p}\end{array}$

$\begin{array}{llllllll}2 & 10-69 & U & 10 & -- & -- & 0 & - \\ 1 & 10-69 & u & 50 & -- & 3 & 0 & p\end{array}$

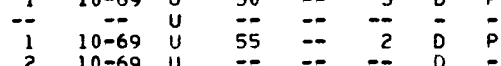

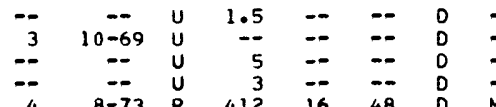

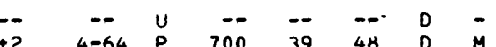

$\begin{array}{cccccccc}+2 & 4-64 & P & 700 & 39 & 48 & D & M \\ -- & -- & U & -- & -- & -- & D & -\end{array}$

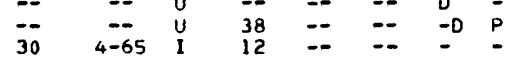

$\begin{array}{rrrrrrrr}-- & -- & U & 1 & -- & -- & D & - \\ 2 & 10-49 & P & 200 & 12 & 120 & D & M \\ 2 & 10-49 & P & 100 & 12 & 120 & - & M \\ 1 & 11-49 & P & 106 & 1 & 120 & - & M \\ -- & -- & U & 15 & -- & - & D & -\end{array}$ 
LOCAL WELL
LATITUDE- ALTILATITUDE-
LONGI TUDE
WELL OWNER OR USER MEAR/ DIAM-IFIN-IOEPTHIUSE FEET WATERMETHOD DIAM-IFIN-IDEPTHIUSE TO BEARING LEVEL TDATE IUSE YIELOT DO ITIME LOG QW DRILLED ETER IISH
(IN) I SWANSEA --CONTINUED

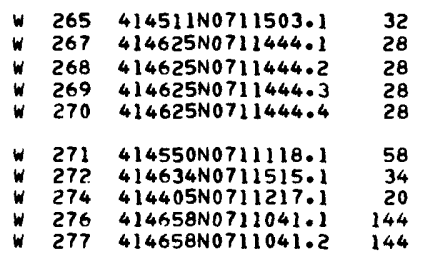

$\times \quad 414709$ N0711109.1 77

$\begin{array}{llll}x & 21 & 414555 \mathrm{~N} 0711406.1 & 66 \\ \mathrm{X} & 22 & 414555 \mathrm{~N} 0711350.1 & 72\end{array}$

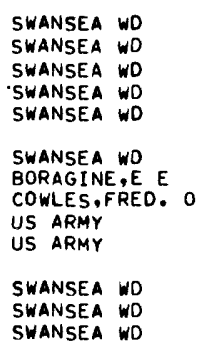

$\begin{array}{ll}1950 & W \\ 1965 & C \\ 1965 & C \\ 1965 & C \\ 1965 & C \\ 1962 & W \\ 1967 & - \\ 1965 & P \\ 1955 & C \\ 1955 & C\end{array}$

\section{2} 1972

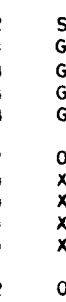

$\begin{array}{rrrr}28 & T & -- & R \\ 36 & W & =- & S \\ 35 & W & =- & 3 R \\ 36 & W & =- & 3 R \\ 35 & W & -- & 36\end{array}$

$\begin{array}{rrrr}23 & 1 & -- & - \\ 82 & W & 24 & --\end{array}$

$\begin{array}{llll}155 & 20 & -- & --\end{array}$

293 W $\quad--\quad--\quad 168$

$\begin{array}{llll}46 & 1 & -- & -- \\ 16 & T & -- & --\end{array}$

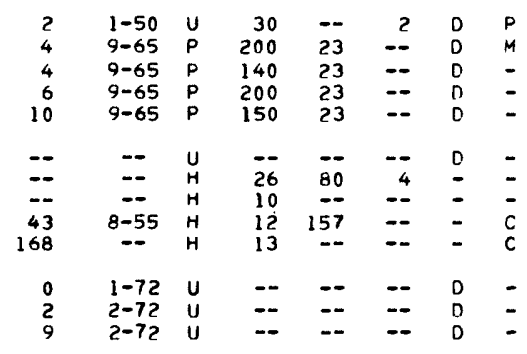

WESTPORT

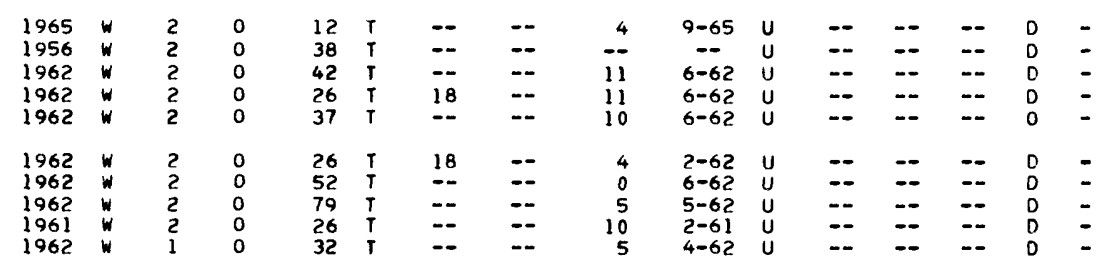

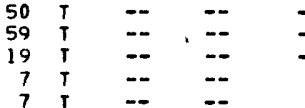

13 413412N0710419.1 -9 MOPW

$14 \quad 413056$ N0710416.1 -11 MDPW

$\begin{array}{llll}15 & 413508 N 0710459.1 & 52 & \text { MDPW } \\ 16 & 413540 \text { N0710452.1 } & 64 & \text { MOPW }\end{array}$

17413630 NO710441.1 67 MOPW

18413635 NO710440.1 72 MOPW

$\begin{array}{rrrr}19 & 413847 N 0710506.1 & 114 & \text { MDPW } \\ 20 & 413802 \text { NO } 710348.1 & 20 & \text { MDPW }\end{array}$

$14140490710654.1 \quad 139$ MOPW

2414048 NO710642.1 148

4 414041N0710613:1 144

$\begin{array}{lll}6 & 414037 N 0710601.1 & 140 \\ 7 & 414035 N 0710556.1 & 140\end{array}$

414032 NOT10552.1

9 414027N0710538.1 163

10414031 No710526.1 186

$11414020 \mathrm{NO} 710513.1 \quad 160$

14 414007N0710449.1 110

15414001 No710440.1 110 $17 \quad 413955 \mathrm{NO} 710.431 .1 \quad 108$ $\begin{array}{lll}19 & 413948 \mathrm{~N} 0710417.1 & 132 \\ 20 & 413939 \mathrm{~N} 0710406.1 & 128\end{array}$ $21413936 \mathrm{~N} 0710355.1 \quad 125$

R 22413931 No710343.1 108 $1413812 \mathrm{~N} 0710748.1 \quad 140$ $413712 N 0710448.1$
$413910 N O T 10439.1$
410 413918N0710534.1 161

7413849 N0710649.1 176 813908 NO710601.1 193 9 413148NO710625.1 55 $11413556 \mathrm{~N} 0710431.150$

13 413916N0710505.1 144 $14 \quad 413756 \mathrm{~N} 0710612.1 \quad 197$ $\begin{array}{ll}412909 \mathrm{NO} 10216.1 & 15 \\ 412919 \mathrm{NO} 10219.1 & 20\end{array}$ 20412925 N0710225.1 $413025 \mathrm{N0} 10320.1 \quad 14$ $413022 N 0710314.1$ $\begin{array}{ll}413010 N 0710230.1 & 10 \\ 413007 N 0710239.1 & 10\end{array}$

413020 N0710313.4 413339 N0710533.1 172 413200 NO 710459.1

39 413241N0710526.1 70 41 413352N0710531.1

180
MDPW

$M D P W$

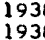

1938

1950

1959

1959

1959

1961

1941

-

1962

1962

1962

MDPW

MDPW

MDPW

MDPW

MDPW

MDPW

MDPW

MDPW

MOPW

MANCLI X,H

DESCHENES, MRS

CYBERT, WALTER

COSTA, ANTONE

PACHECO, ANTONE

MARSTON, WAL TER

PERRY, HENRY F $F$

VITAL, MANUEL $R$

LAUTON.ELLIS

SILVARIUS, N

SILVARIUS, N

$1962 w=0$

1962

1962

1962

1962

1962

1962
1962

1962

--
$1965=$
1963

$1968=$

1966

1966 P

SILVARIUS, N

HORSENECK BEACH 1956 HORSENECK BEACH 1956 HORSENECK BEACH 1956
HORSENECK BEACH 1956

HORSENECK BEACH 1956 W

GILES, HARRY

SOUTHARD, G H

SWARTZ, JOHN
CAMP NOQUOCHOKE

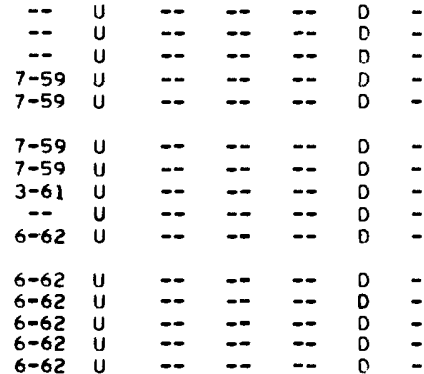

$6-62$ u $\quad=\quad--\quad=-$ $\begin{array}{cccccc}-- & u & = & =- & = & 0 \\ 6-62 & u & = & = & = & 0 \\ 6-62 & 4 & = & = & = & 0\end{array}$

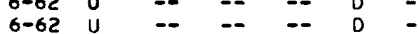

$\begin{array}{lllllll}-- & u & - & -- & - & 0 & -\end{array}$

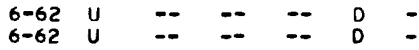

$\begin{array}{rlllll}6-62 & U & - & -- & - & 0 \\ 10-52 & H & - & - & = & -\end{array}$

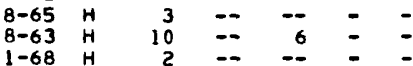

$\begin{array}{lllll}4-66 & H & 4 & - & 2\end{array}$

$\begin{array}{cccccc}8-67 & H & 3 & = & = & = \\ = & H & - & = & = & = \\ = & H & 100 & = & = & =\end{array}$

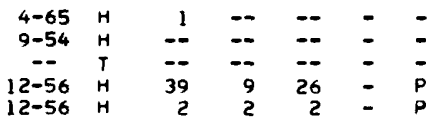

$\begin{array}{rrrrrrr}12-56 & H & 6 & 2 & 20 & - & P \\ 9-56 & U & -- & -- & -- & 0 & - \\ 10-56 & U & 5 & 92 & 5 & 0 & - \\ 11-56 & U & 15 & -- & 4 & 0 & \bar{P} \\ 11-56 & U & 7 & -- & 3 & 0 & P\end{array}$

$11-56$ U $\quad 8 \quad=\quad 3 \quad 0 \quad P$

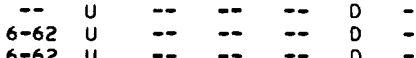


LOCAL

WELL
WELL YEAR/ TIAM-IFIN-IDEPTHIUSE FEET WATER-

PUMPAGE
AUSE YIELDI DD TTIME LOG QW (IN) I I (FT) I RED- MATERIAL (FT) IMEAS-

WESTPORT --CONTINUED
W $42413208 N 0710502.1 \quad 120$ 46 413353N0710711.1

$47 \quad 413321$ 1N0710708.1 50 413328N0710724. 51413019 N0710710.

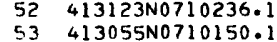

55 413715N0710341.1 $59 \quad 413146$ N0710411.1 50

6? 413443N0710302.1 120 63 413857N0710454.1 $64 \quad 413814 N 0710315$.

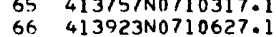

67 413934N0710627.1 $68 \quad 414026 \mathrm{N0710635.}$ 70 413854N0710703.1 72 414011N0710636.1 195

$74 \quad 413136$ NO710433.1 $75413452 N 0710231.1$ 77413910 NO710716.1 80 414004N0710619.1

$81413808 \mathrm{NO} 10301.1$ $83414118 N 0710539.1$ $85 \quad 414002 N 0710640.1$

$86414002 \mathrm{N0710632.1}$ B7 413830N0710353.1 RB 413815N0710254. $89413915 N 0710428.1$
$90 \quad 414048 N 0710613.1$ $91413926 \mathrm{N0710715.1}$ $92414013 N 0710640$. $95413110 N 0710424$. $96 \quad 413255 N 0710533.1$

$98 \quad 413444 N 0710529.1$
$-99413234 N 0710516.1$ $100413136 N 0710446.1$ $101413412 N 0710436.1$ - 163 413202N0710408.

W 105 413309N0710411. W $106 \quad 413415 N 0710352$. $107413118 N 0710430.1$
$108413132 N 0710436.1$ $108413132 N 0710436.1$
$W \quad 109 \quad 413538 N 0710437.1$ W $111413216 \mathrm{N071040S.1} 55$

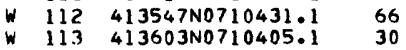
$w 114 \quad 413952 N 0710612.1 \quad 175$

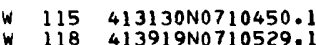
W $120 \quad 413914 N 0710018.1$

* $122413721 N 0710351.1$ W $124 \quad 413436 N 0710530.1$ $W \quad 125 \quad 413425 N 0710629.1$ W 127 413641N0710305.1

$128 \quad 413611$ N0710315. W 129 413106N0710706. $W \quad 129413106 N 0710706.1$ $W 131$
$W 1324047 N 0710643.1$
$W 13716 N 0710328.1$ $43413143 \mathrm{~N} 0710439.1$ $44 \quad 413318 N 0710737.1$ SR 413151N0710443.1 102 $61413709 \mathrm{~N} 0710312.163$ $82413835 N 0710303.1$

W $110 \quad 413302$ N0710415.1 101 * $119413223 N 0710354$.
BIRKETT,WILLIAM 1966 MARTINEAU,W 1966 LEACH,GEORGE JR $2969=$
PERRY,JOSEPH
SIS65 SISSON, SUSAN M 1968

WILBOUR,F C JR. 1963 PERRY, JOSEPH SR 1966 $\begin{array}{ll}\text { BOYLE, EDWARD } & 1965 \\ \text { COBURN,WILLIAM } & 1966\end{array}$ GIFFORD,RICHARD $1966=$

FRANCE, DONALD W 1964 DEKNATEL,F B 1966 $\begin{array}{lll}\text { PIERCE A ALLEN S } & 1964 \\ \text { WAITE, ERNEST B. } & 1965\end{array}$ BOAN,ALTON A 1966

TRIPP,HOWARDS 1964 CDSTA,RICHARD 1968 WILEY,WILLIAM 1963 THE GARDEN SHOP $1963=$

BDOTHROYD,DORIS 1963 BRIGHTMAN, HENRY SLATER, CLYDE $\begin{array}{ll}\text { SIMONIN,NORMAN } & 1967 \\ \text { BASSETT,G. } & 1966\end{array}$

BABCOCK,CHESTER 1965 WODD,RAYMOND W 1966 QUINN, JAMES LA PLANTE, R W $1966=$

CHRISTIANSEN,A 1966 RENAUD,BRYAN J 1965 8LOSSOM-PETTEY 1966 PACHECO,JOSEPH 1964 ANDRAOE.VALERIO 1963

SZELAG,WALTER 1963 $\begin{array}{ll}\text { LAPRISE, ALBERT } & 1963= \\ \text { LEVESQUE,M D } & 1963=\end{array}$ LEVESQUE,M D , 1963 P $\begin{array}{lll}\text { PERRY,RUSSELL J } 1969 & \text { P } \\ \text { ZEMBO.LDUIS J } 1964=\end{array}$

BDULLARD,F M 1964 COSTA,THOMAS M 1965 OFNTON,THEODORE 1963 $\begin{array}{ll}\text { TRIPP-PEARSDN } & 1965= \\ \text { ROUSSEAU,HENRI } & 1966=\end{array}$

WOOD, KENNETH E

BUSH, GEOFFREY

ALEEN, HAROLO A 1964 -

EARLE, SYLVIA S 1963

MATHEWS,K C

BOAN, ALTON A $\quad 1964$

SOWLE, MARY EST. $1964=$

SCHOFIELD,R.

BRIGHTMAN,L A

KEEFE, THOMAS W

PERRY -KIRBY

MURRAY,DORIS

PERREIRA,R

WOYTASZEK, E

SOREL,RUDOLF N 1969

MARTIN,MANUEL 1970 -

WESTPORT LIBRAR $1970 \quad P$

BOWMAN,LUTHER 81966

LEWIS, MANUEL

MARTENS,CLAIRE 1965

SIMOES, ANTONE $J 1964$

ZALEWSKI, S C $1966=$

SOUZA,RDBERT P 1964 -

$\begin{array}{ll}\text { BLISS, LESLIE T } & 1968= \\ \text { DOSVAIS,MANUEL } & 1964=\end{array}$
FRISCH,DAVIO H

\begin{tabular}{|c|c|c|c|}
\hline 6 & $x$ & 208 & $w$ \\
\hline 6 & $x$ & 147 & $w$ \\
\hline $\begin{array}{l}6 \\
6\end{array}$ & $\begin{array}{l}x \\
x\end{array}$ & $\begin{array}{r}137 \\
85\end{array}$ & $w$ \\
\hline 6 & $x$ & 135 & $w$ \\
\hline 6 & $x$ & 100 & $w$ \\
\hline 6 & $x$ & 160 & $w$ \\
\hline 6 & $x$ & 130 & $\hat{w}$ \\
\hline 6 & $x$ & 460 & $W$ \\
\hline 6 & $x$ & 67 & $W$ \\
\hline 6 & $x$ & 202 & $W$ \\
\hline 6 & $x$ & 2SS & 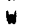 \\
\hline 6 & $x$ & 110 & $w$ \\
\hline 6 & $x$ & 232 & 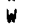 \\
\hline 6 & $x$ & 104 & $W$ \\
\hline 6 & $x$ & 595 & $w$ \\
\hline 6 & $x$ & 160 & $w$ \\
\hline 6 & $x$ & 94 & $W$ \\
\hline 6 & $x$ & 97 & $W$ \\
\hline 6 & $x$ & 170 & $w$ \\
\hline 6 & $x$ & 88 & $W$ \\
\hline 6 & $x$ & 109 & $w$ \\
\hline 6 & $x$ & 117 & $w$ \\
\hline 6 & $x$ & 152 & $v$ \\
\hline 6 & $x$ & 238 & $w$ \\
\hline 6 & $x$ & 127 & $w$ \\
\hline 6 & $x$ & 328 & $W$ \\
\hline 6 & $x$ & 108 & $w$ \\
\hline 6 & $x$ & 134 & $w$ \\
\hline 6 & $x$ & 96 & $w$ \\
\hline 6 & $x$ & 88 & $W$ \\
\hline 6 & $x$ & 186 & $w$ \\
\hline 6 & $x$ & 104 & $w$ \\
\hline $\begin{array}{l}6 \\
6\end{array}$ & $x$ & 98 & $w$ \\
\hline & & & \\
\hline 6 & $x$ & 138 & $w$ \\
\hline 6 & $x$ & 80 & $w$ \\
\hline 6 & $x$ & 187 & 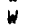 \\
\hline 6 & $x$ & 110 & 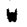 \\
\hline 6 & $x$ & 84 & $\ddot{*}$ \\
\hline 6 & $x$ & 90 & $w$ \\
\hline 6 & $x$ & 127 & $w$ \\
\hline 6 & $x$ & 100 & $w$ \\
\hline 6 & $x$ & 150 & W \\
\hline 6 & $x$ & 210 & $W$ \\
\hline 6 & $x$ & 95 & $w$ \\
\hline 6 & $x$ & 150 & W \\
\hline 6 & $x$ & 165 & $W$ \\
\hline 6 & $x$ & $=$ & 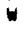 \\
\hline 6 & $x$ & 79 & $w$ \\
\hline 6 & $x$ & 208 & $w$ \\
\hline 6 & $x$ & 103 & $W$ \\
\hline 6 & $x$ & 202 & $w$ \\
\hline 6 & $x$ & 100 & $W$ \\
\hline 6 & $x$ & 142 & $w$ \\
\hline 6 & $x$ & 189 & $w$ \\
\hline 6 & $x$ & 83 & $\tilde{\omega}$ \\
\hline 6 & $x$ & 432 & $w$ \\
\hline 6 & $\hat{x}$ & 115 & $w$ \\
\hline 6 & $x$ & 97 & $w$ \\
\hline 6 & $x$ & 95 & $w$ \\
\hline 6 & $x$ & 142 & $\omega$ \\
\hline 6 & $x$ & 295 & 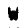 \\
\hline 6 & $x$ & 98 & $\bar{W}$ \\
\hline 6 & $x$ & 100 & $w$ \\
\hline 6 & $x$ & 155 & $w$ \\
\hline 6 & $x$ & 88 & $W$ \\
\hline 6 & $x$ & 96 & $\omega$ \\
\hline 6 & $\bar{x}$ & 470 & $W$ \\
\hline 6 & $x$ & 98 & $\omega$ \\
\hline 6 & $x$ & 98 & $w$ \\
\hline 6 & $\hat{x}$ & 161 & $w$ \\
\hline 6 & $x$ & 85 & 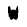 \\
\hline 6 & $x$ & 250 & $w$ \\
\hline 6 & $x$ & 130 & $W$ \\
\hline
\end{tabular}

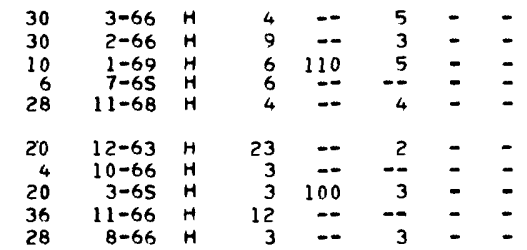

$10-64 \quad \mathrm{H}$ 4-66 H $\begin{array}{ll}10-64 & H \\ 12-65 & H\end{array}$ $\begin{array}{rr}12-65 & H \\ 9-66 & H\end{array}$

$12-64 \mathrm{~N}$ $\begin{array}{rr}11-68 & H \\ 8-63 & H\end{array}$ $11-68$

9-63 H $1-64 \quad H$ $\begin{array}{ll}5-66 & H \\ 1-67 & \end{array}$ $\begin{array}{ll}1-67 & H \\ 5-66 & H\end{array}$

8-65 H $5-66$
$H$ $\begin{array}{ll}12-63 & H \\ 10-66 & H\end{array}$ $10-66$
$8-64$

2-66 H $3-65 \quad H$ $\begin{array}{ll}8-66 & H \\ 1-64 & H\end{array}$ $8-63 H$ $\begin{array}{lllll}60 & - & 18 & 10-63 & H \\ 35 & - & 23 & 10-63 & H \\ 47 & - & 7 & 11-63 & H\end{array}$ $31=$

4-64

$\begin{array}{ll}20 & =- \\ 30 & =- \\ 16 & =- \\ 48 & =-\end{array}$

$9-64 \quad H$ $9-63$
$9-65$ 9-66

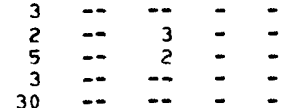

$\begin{array}{ccc}8 & -- & = \\ 2 & -- & = \\ -3 & \cdots & = \\ 3 & - & -\end{array}$

3
5
5
2
6
4
5
0

75
$=-$
$=-$
$=$
$\therefore-$
$-\therefore$
48

$\begin{array}{rr}12 & - \\ 6 & = \\ 12 & \cdots \\ 10 & -\end{array}$

8
4
3
20
15

$\because$

8
6
5
4
4

$\begin{array}{rr}-- & 0 \\ = & -- \\ = & 2 \\ -- & 4\end{array}$
$56 \quad=$

$\begin{array}{ll}7-66 & H \\ 5-66 & H\end{array}$

2-64 H

$8-66$
$10-63$

$6-66 \mathrm{H}$

20 --

27
40
15
30

$8-64$
$6-66$

$6-66$
$2-64$

$10-63 \cdot \mathrm{H}$

9-63 H

4-65 $\mathrm{H}$

$3-67 \quad H$
$4-66$

$4-66$
$12-65$

20

150

$1-69$
$1-68$

$11=70 \quad H$

$9-70$

3
1.5
8
3
20
3
5
15
4
36

$=-8$

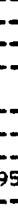

30
4
6
6
7

$\begin{array}{ll}34 & = \\ 40 & =- \\ 12 & =\end{array}$

$\begin{array}{ll}7-64 & H \\ 0-65 & H\end{array}$

$11-64$

$\begin{array}{rrrrr}11 & -- & 20 & 11-64 & H \\ 24 & -- & 20 & 3-66 & H\end{array}$

40 -..

$10-64$
$12-68$

$12-68$
$4-64$ 


\begin{tabular}{|c|c|c|c|c|c|c|c|c|c|c|c|c|c|c|c|c|c|c|c|c|}
\hline \multirow{2}{*}{\multicolumn{2}{|c|}{$\begin{array}{l}\text { LOCAL } \\
\text { WELLL } \\
\text { NUMBER }\end{array}$}} & \multirow[b]{2}{*}{$\begin{array}{l}\text { LATITUDE- } \\
\text { LONGITUDE }\end{array}$} & \multirow[b]{2}{*}{$\begin{array}{l}\text { ALTI- } \\
\text { TUDE } \\
\text { OF LSD } \\
\text { (FT) }\end{array}$} & \multirow[b]{2}{*}{ OWNER OR USER } & \multicolumn{6}{|c|}{ WELL } & \multirow[b]{2}{*}{$\begin{array}{l}\text { FEET } \\
\text { TO } \\
\text { BED- } \\
\text { ROCK }\end{array}$} & \multirow[b]{2}{*}{$\begin{array}{l}\text { WATER- } \\
\text { BEARING } \\
\text { MATERIAL }\end{array}$} & \multicolumn{3}{|c|}{ WATER } & \multicolumn{3}{|c|}{ PUMPAGE } & \multirow[b]{2}{*}{ LOG } & \multirow[b]{2}{*}{$2 W$} \\
\hline & & & & & \multicolumn{2}{|c|}{$\begin{array}{l}\text { METHOD } \\
\text { DRILLED }\end{array}$} & \multicolumn{4}{|c|}{$\begin{array}{l}\text { DIAM-IFIN-IDEPTHIUSE } \\
\text { EIER IISH IS } \\
\text { (IN) I }\end{array}$} & & & \multirow[t]{2}{*}{$\begin{array}{l}\overline{\text { LEVEL }} \\
(F T)\end{array}$} & \multicolumn{2}{|l|}{$\begin{array}{l}\text { TOATE I } \\
\text { IMEAS- I } \\
\text { IURED I }\end{array}$} & \multicolumn{3}{|c|}{\begin{tabular}{l|l|l} 
YIELD & DDIME \\
(GPM)। (FT) I(HR)
\end{tabular}} & & \\
\hline \multicolumn{20}{|c|}{ WESTPORT - CONTINUED } & \\
\hline $\begin{array}{c}w \\
w \\
w \\
w \\
w\end{array}$ & $\begin{array}{l}134 \\
135 \\
136 \\
137 \\
138\end{array}$ & $\begin{array}{l}412948 N 0710703.1 \\
413716 N 0710218.1 \\
413730 N 0710341.1 \\
413323 N 0710320.1 \\
413308 N 0710314.1\end{array}$ & $\begin{array}{r}20 \\
131 \\
40 \\
15 \\
15\end{array}$ & $\begin{array}{l}\text { FEENER, EDNA F } \\
\text { DUTRA, } S \text { S } \\
\text { CLARK - JOHANSEN } \\
\text { SPOONER, RALPH } \\
\text { VIEIRA, } J\end{array}$ & $\begin{array}{l}1965 \\
1964 \\
1965 \\
1964 \\
1963\end{array}$ & $=$ & $\begin{array}{l}6 \\
6 \\
6 \\
6 \\
6\end{array}$ & $\begin{array}{l}x \\
x \\
x \\
x \\
x\end{array}$ & $\begin{array}{r}60 \\
94 \\
265 \\
72 \\
76\end{array}$ & $w$ & $\begin{array}{l}30 \\
40 \\
28 \\
28 \\
20\end{array}$ & $\begin{array}{l}-- \\
= \\
--\end{array}$ & $\begin{array}{l}30 \\
20 \\
18 \\
10 \\
15\end{array}$ & $\begin{array}{r}6-65 \\
11-64 \\
10-65 \\
8-64 \\
7-63\end{array}$ & $\begin{array}{l}\mathrm{H} \\
\mathrm{H} \\
\mathrm{H} \\
\mathrm{H} \\
\mathrm{H}\end{array}$ & $\begin{array}{r}3 \\
7 \\
1 \\
10 \\
6\end{array}$ & $\begin{array}{l}-- \\
\ddot{-} \\
=\end{array}$ & $\begin{array}{r}3 \\
-- \\
-- \\
8 \\
2\end{array}$ & $\begin{array}{l}- \\
=\end{array}$ & $\begin{array}{l}- \\
\overline{-}\end{array}$ \\
\hline $\begin{array}{c}w \\
w \\
w \\
w \\
w\end{array}$ & $\begin{array}{l}139 \\
140 \\
141 \\
143 \\
144\end{array}$ & $\begin{array}{l}413033 N 0710636.1 \\
413129 N 0710611.1 \\
413758 N 0710411.1 \\
413940 N 0710423.1 \\
413850 N 0710412.1\end{array}$ & $\begin{array}{r}35 \\
78 \\
99 \\
110 \\
68\end{array}$ & $\begin{array}{l}\text { PHINNEY, EDWARD } \\
\text { MEADER, DANIEL E } \\
\text { TRIPP, CALVIN F } \\
\text { WESTPORT TOWN } \\
\text { WESTPORT TOWN }\end{array}$ & $\begin{array}{l}1969 \\
1963 \\
1965 \\
1964 \\
1964\end{array}$ & $\overline{-}$ & $\begin{array}{l}6 \\
6 \\
6 \\
2 \\
2\end{array}$ & $\begin{array}{l}x \\
x \\
x \\
0 \\
0\end{array}$ & $\begin{array}{r}126 \\
138 \\
92 \\
41 \\
27\end{array}$ & $\begin{array}{l}w \\
w \\
w \\
T \\
T\end{array}$ & $\begin{array}{l}28 \\
40 \\
39 \\
-- \\
--\end{array}$ & $\begin{array}{l}=- \\
\ddot{-} \\
--\end{array}$ & $\begin{array}{l}10 \\
20 \\
30 \\
--\end{array}$ & $\begin{array}{r}3-69 \\
11-63 \\
3-65 \\
-- \\
--\end{array}$ & $\begin{array}{l}H \\
H \\
H \\
U \\
U\end{array}$ & $\begin{array}{r}5 \\
4 \\
7 \\
-- \\
--\end{array}$ & $\begin{array}{l}=- \\
=- \\
=-\end{array}$ & $\begin{array}{r}3 \\
2 \\
4 \\
-- \\
--\end{array}$ & $\begin{array}{l}- \\
\bar{D} \\
0\end{array}$ & $\begin{array}{l}\overline{-} \\
\overline{-}\end{array}$ \\
\hline $\begin{array}{c}w \\
w \\
w \\
w\end{array}$ & $\begin{array}{l}145 \\
147 \\
148 \\
151 \\
152\end{array}$ & $\begin{array}{l}413806 N 0710342.1 \\
413812 N 0710358.1 \\
413746 N 0710334.1 \\
413718 N 0710338.1 \\
413754 N 0710252.1\end{array}$ & $\begin{array}{r}45 \\
40 \\
9 \\
8 \\
42\end{array}$ & $\begin{array}{l}\text { WESTPORT TOWN } \\
\text { WESTPORT TOWN } \\
\text { WESTPORT TOWN } \\
\text { WESTPORT TOWN } \\
\text { WESTPORT TOWN }\end{array}$ & & $\begin{array}{l}w \\
w \\
w \\
w\end{array}$ & $\begin{array}{l}2 \\
2 \\
2 \\
2 \\
2\end{array}$ & $\begin{array}{l}0 \\
0 \\
0 \\
0 \\
0\end{array}$ & $\begin{array}{l}18 \\
22 \\
36 \\
41 \\
18\end{array}$ & $\begin{array}{l}T \\
T \\
T \\
T \\
T\end{array}$ & $\begin{array}{l}= \\
= \\
=\end{array}$ & $\begin{array}{l}\ddot{-} \\
25 \\
-- \\
25\end{array}$ & $\frac{--}{-2}$ & $\begin{array}{c}= \\
12-64 \\
-- \\
12-64\end{array}$ & $\begin{array}{l}u \\
u \\
u \\
u \\
u\end{array}$ & $\begin{array}{l}-- \\
20 \\
10\end{array}$ & $\begin{array}{l}= \\
= \\
= \\
--\end{array}$ & $\begin{array}{l}= \\
\pm \\
=\end{array}$ & $\begin{array}{l}D \\
D \\
D \\
D \\
D\end{array}$ & ! \\
\hline $\begin{array}{c}w \\
w \\
w \\
w\end{array}$ & $\begin{array}{l}153 \\
154 \\
155 \\
156 \\
157\end{array}$ & $\begin{array}{l}413735 N 0710328.1 \\
413741 N 0710321.1 \\
413755 N 0710326.1 \\
413631 N 0710327.1 \\
413954 N 0710718.1\end{array}$ & $\begin{array}{r}34 \\
30 \\
34 \\
42 \\
143\end{array}$ & $\begin{array}{l}\text { WESTPORT TOWN } \\
\text { WESTPORT TOWN } \\
\text { WESTPORT TOWN } \\
\text { WESTPORT TOWN } \\
\text { WESTPORT TOWN }\end{array}$ & $\begin{array}{l}1964 \\
1964 \\
1964 \\
1964 \\
1964\end{array}$ & $\begin{array}{c}w \\
w \\
w \\
w\end{array}$ & $\begin{array}{l}2 \\
2 \\
2 \\
2 \\
2\end{array}$ & $\begin{array}{l}0 \\
0 \\
0 \\
0 \\
0\end{array}$ & $\begin{array}{l}47 \\
37 \\
36 \\
48 \\
13\end{array}$ & $\begin{array}{l}T \\
T \\
T \\
T \\
T\end{array}$ & $\begin{array}{l}= \\
\square \\
= \\
-\end{array}$ & $\begin{array}{l}9 \mathrm{~S} \\
\square- \\
=- \\
-\end{array}$ & $\begin{array}{l}23 \\
18 \\
-26 \\
26\end{array}$ & $\begin{array}{c}12-64 \\
12-64 \\
-- \\
12-64 \\
--\end{array}$ & $\begin{array}{l}u \\
u \\
u \\
u \\
u\end{array}$ & $\begin{array}{l}20 \\
-- \\
--\end{array}$ & 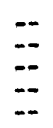 & $\begin{array}{l}-- \\
\ddot{-} \\
--\end{array}$ & $\begin{array}{l}0 \\
D \\
0 \\
0 \\
D\end{array}$ & $\begin{array}{l}\overline{-} \\
\overline{-}\end{array}$ \\
\hline $\begin{array}{l}w \\
w \\
w \\
w\end{array}$ & $\begin{array}{l}158 \\
159 \\
160 \\
161 \\
162\end{array}$ & $\begin{array}{l}413617 N 0710358.1 \\
413646 N 0710329.1 \\
413950 N 0710523.1 \\
413725 N 0710332.1 \\
413616 N 0710803.1\end{array}$ & $\begin{array}{r}12 \\
13 \\
140 \\
8 \\
139\end{array}$ & $\begin{array}{l}\text { WESTPORT TOWN } \\
\text { WESTPORT TOWN } \\
\text { WESTPORT TOWN } \\
\text { WESTPORT TOWN } \\
\text { WESTPORT TOWN }\end{array}$ & $\begin{array}{l}1965 \\
1965 \\
1965 \\
1965 \\
1965\end{array}$ & $\begin{array}{l}w \\
w \\
w \\
w\end{array}$ & $\begin{array}{l}2 \\
2 \\
2 \\
2 \\
2\end{array}$ & $\begin{array}{l}0 \\
\mathbf{S} \\
0 \\
0 \\
0\end{array}$ & $\begin{array}{l}31 \\
38 \\
36\end{array}$ & $\begin{array}{l}T \\
T \\
T \\
T \\
T\end{array}$ & $\begin{array}{l}-- \\
= \\
--\end{array}$ & $\begin{array}{l}-- \\
2 R \\
-- \\
--\end{array}$ & $\begin{array}{r}-- \\
-- \\
--\end{array}$ & $\begin{array}{l}-- \\
2-65 \\
2-65 \\
=-\end{array}$ & $\begin{array}{l}u \\
u \\
u \\
u \\
u\end{array}$ & $\begin{array}{r}-- \\
10 \\
273 \\
--\end{array}$ & $\begin{array}{l}-- \\
12 \\
--\end{array}$ & $\frac{-}{142}$ & $\begin{array}{l}D \\
D \\
D \\
D \\
D\end{array}$ & $\begin{array}{l}- \\
\overline{-} \\
\overline{-}\end{array}$ \\
\hline $\begin{array}{c}w \\
w \\
w \\
w\end{array}$ & $\begin{array}{l}163 \\
164 \\
165 \\
166 \\
167\end{array}$ & $\begin{array}{l}413240 N 0710306 \\
413337 N 0710450 \\
413021 N 0710300 \\
413024 N 0710301 \\
413025 N 0710259\end{array}$ & $\begin{array}{l}10 \\
72 \\
10 \\
12 \\
11\end{array}$ & $\begin{array}{l}\text { WESTPORT TOWN } \\
\text { WESTPDRT TOWN } \\
\text { HORSENECK BEACH } \\
\text { HORSENECK BEACH } \\
\text { HORSENECK BEACH }\end{array}$ & $\begin{array}{l}1965 \\
1965 \\
1958 \\
1958 \\
1958\end{array}$ & $\begin{array}{l}w \\
w \\
c \\
c \\
c\end{array}$ & $\begin{array}{r}2 \\
2 \\
12 \\
12 \\
12\end{array}$ & $\begin{array}{l}0 \\
0 \\
G \\
G \\
G\end{array}$ & $\begin{array}{l}2 \\
2 \\
1 \\
2 \\
1\end{array}$ & $\begin{array}{c}T \\
T \\
w \\
w \\
w\end{array}$ & $\begin{array}{l}=- \\
\ddot{-} \\
=\end{array}$ & $\begin{array}{l}\ddot{--} \\
2 R \\
2 S \\
2 S\end{array}$ & $\begin{array}{r}-- \\
4 \\
7\end{array}$ & $\begin{array}{c}-- \\
-- \\
5-58 \\
5-58 \\
5-58\end{array}$ & $\begin{array}{l}u \\
u \\
T \\
T \\
T\end{array}$ & $\begin{array}{l}-- \\
20 \\
20 \\
20\end{array}$ & $\begin{array}{r}-- \\
-- \\
2 \\
2 \\
4\end{array}$ & $\begin{array}{r}-- \\
-- \\
8 \\
8 \\
8\end{array}$ & $\begin{array}{l}D \\
0\end{array}$ & $\bar{M}$ \\
\hline $\begin{array}{l}w \\
w \\
w \\
w\end{array}$ & $\begin{array}{l}168 \\
169 \\
170 \\
171 \\
172\end{array}$ & $\begin{array}{l}413025 N 0710256.1 \\
413025 N 0710252.1 \\
413024 N 0710249.1 \\
413023 N 0710247.1 \\
413021 N 0710244.1\end{array}$ & $\begin{array}{l}5 \\
8 \\
9 \\
6 \\
7\end{array}$ & $\begin{array}{l}\text { HORSENECK BEACH } \\
\text { HORSENECK BEACH } \\
\text { HORSENECK BEACH } \\
\text { MORSENECK BEACH } \\
\text { HORSENECK BEACH }\end{array}$ & $\begin{array}{l}1958 \\
1958 \\
1958 \\
1958 \\
1958\end{array}$ & $\begin{array}{l}c \\
C \\
c \\
c \\
c\end{array}$ & $\begin{array}{l}12 \\
12 \\
12 \\
12 \\
12\end{array}$ & $\begin{array}{l}G \\
G \\
G \\
G \\
G\end{array}$ & $\begin{array}{l}19 \\
19 \\
17 \\
16 \\
15\end{array}$ & $\underset{w}{w} w$ & $\begin{array}{l}= \\
= \\
=\end{array}$ & $\begin{array}{l}2 R \\
2 R \\
2 R \\
2 R \\
2 R\end{array}$ & $\begin{array}{l}6 \\
6 \\
6 \\
5 \\
4\end{array}$ & $\begin{array}{l}5-58 \\
5-58 \\
5-58 \\
5-58 \\
5-58\end{array}$ & $\begin{array}{l}T \\
T \\
T \\
T \\
T\end{array}$ & $\begin{array}{l}20 \\
20 \\
20 \\
20 \\
20\end{array}$ & $\begin{array}{l}4 \\
2 \\
2 \\
2 \\
2\end{array}$ & $\begin{array}{l}8 \\
8 \\
8 \\
8 \\
8\end{array}$ & $\begin{array}{l}D \\
D \\
D\end{array}$ & $\begin{array}{l}M \\
M \\
M \\
M \\
M\end{array}$ \\
\hline $\begin{array}{l}w \\
w \\
w \\
w\end{array}$ & $\begin{array}{l}173 \\
174 \\
175 \\
176 \\
177\end{array}$ & $\begin{array}{l}413020 N 0710242.1 \\
413018 N 0710239.1 \\
413016 N 0710236.1 \\
413015 N 0710234.1 \\
413013 N 0710233.1\end{array}$ & $\begin{array}{l}8 \\
8 \\
8 \\
8 \\
8\end{array}$ & $\begin{array}{l}\text { HORSENECK } \text { BEACH } \\
\text { HORSENECK BEACH } \\
\text { HORSENECK } \text { BEACH } \\
\text { HORSENECK BEACH } \\
\text { HORSENECK BEACH }\end{array}$ & $\begin{array}{l}1957 \\
1957 \\
1957 \\
1957 \\
1957\end{array}$ & $\begin{array}{l}w \\
w \\
w \\
w \\
w\end{array}$ & $\begin{array}{l}2 \\
2 \\
2 \\
2 \\
2\end{array}$ & $\begin{array}{l}5 \\
5 \\
5 \\
5 \\
5\end{array}$ & $\begin{array}{l}= \\
=- \\
=\end{array}$ & $\begin{array}{l}T \\
T \\
T \\
T \\
T\end{array}$ & $\begin{array}{l}= \\
=- \\
= \\
--\end{array}$ & $\begin{array}{l}-- \\
m \\
--\end{array}$ & $\begin{array}{l}4 \\
4 \\
4 \\
4 \\
4\end{array}$ & $\begin{array}{l}12-57 \\
12-57 \\
12-57 \\
12-57 \\
12-57\end{array}$ & $\begin{array}{l}u \\
u \\
u \\
u \\
u\end{array}$ & $\begin{array}{l}-- \\
-- \\
--\end{array}$ & $\begin{array}{l}-- \\
-- \\
--\end{array}$ & $\begin{array}{l}-- \\
-- \\
--\end{array}$ & $\begin{array}{l}\text { - } \\
\text { - }\end{array}$ & $\begin{array}{l}- \\
\overline{-}\end{array}$ \\
\hline$w$ & $\begin{array}{l}178 \\
179 \\
180 \\
181 \\
183\end{array}$ & $\begin{array}{l}413011 N 0710230.1 \\
413010 N 0710230.2 \\
413954 N 0710507.1 \\
414036 N 0710518.1 \\
414006 N 0710643.1\end{array}$ & $\begin{array}{r}8 \\
8 \\
152 \\
175 \\
144\end{array}$ & $\begin{array}{l}\text { MORSENECK BEACH } \\
\text { MORSENECK BEACH } \\
\text { MILLER,H J } \\
\text { TRIPP, J H SR } \\
\text { FREOERICK,D }\end{array}$ & $\begin{array}{l}1957 \\
1957 \\
1964 \\
1965 \\
1963\end{array}$ & $\begin{array}{l}w \\
w \\
p \\
p\end{array}$ & $\begin{array}{l}2 \\
2 \\
6 \\
6 \\
6\end{array}$ & $\begin{array}{l}5 \\
5 \\
x \\
x \\
x\end{array}$ & $\begin{array}{r}-- \\
90 \\
320 \\
71\end{array}$ & $\begin{array}{l}T \\
T \\
w \\
w \\
w\end{array}$ & $\begin{array}{l}-- \\
-2 \\
55 \\
31 \\
41\end{array}$ & $\begin{array}{l}\ddot{-} \\
\ddot{-} \\
--\end{array}$ & $\begin{array}{l}4 \\
4 \\
30 \\
10 \\
10\end{array}$ & $\begin{array}{r}12-57 \\
12-57 \\
6-64 \\
2-65 \\
12-63\end{array}$ & H & $\begin{array}{r}-- \\
10 \\
1 \\
7\end{array}$ & $\begin{array}{l}-- \\
80 \\
30\end{array}$ & $\frac{-}{10}$ & $=$ & $\begin{array}{l}\overline{-} \\
\vdots\end{array}$ \\
\hline $\begin{array}{l}w \\
w \\
w \\
w\end{array}$ & $\begin{array}{l}184 \\
185 \\
186 \\
187 \\
192\end{array}$ & $\begin{array}{l}413925 N 07106 \\
413850 N 07106 \\
413823 N 07106 \\
413904 N 07105 \\
413004 N 07102\end{array}$ & $\begin{array}{l}155 \\
192 \\
183 \\
135 \\
20\end{array}$ & $\begin{array}{l}\text { ROY J P \& SON } \\
\text { PERRY JOHN J } \\
\text { FERREIRA,W } \\
\text { ESTACIO, JOSEPH } \\
\text { HORSENECK BEACH }\end{array}$ & $\begin{array}{l}1970 \\
1965 \\
1965 \\
1965 \\
1972\end{array}$ & $\begin{array}{l}P \\
\bar{p} \\
\mathbf{W}\end{array}$ & $\begin{array}{l}6 \\
6 \\
6 \\
6 \\
2\end{array}$ & $\begin{array}{l}x \\
x \\
x \\
x \\
s\end{array}$ & $\begin{array}{r}140 \\
258 \\
130 \\
100 \\
20\end{array}$ & $\begin{array}{c}w \\
w \\
w \\
w\end{array}$ & $\begin{array}{l}50 \\
30 \\
38 \\
40 \\
--\end{array}$ & $\begin{array}{l}-- \\
-- \\
2 R\end{array}$ & $\begin{array}{r}8 \\
15 \\
15 \\
-- \\
4\end{array}$ & $\begin{array}{l}2-70 \\
7-65 \\
4=65 \\
-- \\
1-73\end{array}$ & $\begin{array}{l}H \\
H \\
H \\
H\end{array}$ & $\begin{array}{r}2 \\
3 \\
7 \\
2 \\
50\end{array}$ & $\begin{array}{l}=- \\
=- \\
=\end{array}$ & 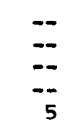 & $\begin{array}{l}- \\
\overline{0}\end{array}$ & $\begin{array}{l}\overline{-} \\
\overline{-}\end{array}$ \\
\hline $\begin{array}{l}W \\
W \\
W \\
W \\
W\end{array}$ & $\begin{array}{l}194 \\
196 \\
197 \\
198 \\
199\end{array}$ & $\begin{array}{l}413518 N 0710524.1 \\
414102 N 0710639.1 \\
414051 N 0710613.1 \\
413845 N 0710448.1 \\
413810 N 0710422.1\end{array}$ & $\begin{array}{l}134 \\
140 \\
149 \\
130 \\
101\end{array}$ & $\begin{array}{l}\text { TRECIDA,RICHARO } \\
\text { SAMPSON FARM } \\
\text { SAMPSON FARM } \\
\text { RODRIGUES,A. } \\
\text { DIAS,RAYMOND }\end{array}$ & $\begin{array}{c}1964 \\
1965 \\
-- \\
1965 \\
1972\end{array}$ & $\begin{array}{l}\bar{w} \\
c \\
\bar{p}\end{array}$ & $\begin{array}{l}6 \\
2 \\
6 \\
6 \\
6\end{array}$ & $\begin{array}{l}x \\
s \\
x \\
x \\
x\end{array}$ & $\begin{array}{r}98 \\
60 \\
100 \\
125 \\
125\end{array}$ & $\begin{array}{l}w \\
T \\
w \\
w \\
w\end{array}$ & $\begin{array}{l}60 \\
35 \\
45 \\
45\end{array}$ & 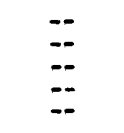 & $\begin{array}{l}30 \\
13 \\
-- \\
30 \\
--\end{array}$ & $\begin{array}{c}9-64 \\
9-65 \\
-- \\
4-65 \\
--\end{array}$ & $\begin{array}{l}H \\
U \\
I \\
H \\
H\end{array}$ & $\begin{array}{r}4 \\
2 \\
15 \\
2 \\
3\end{array}$ & $\begin{array}{l}80 \\
=- \\
=- \\
--\end{array}$ & $\begin{array}{l}5 \\
=- \\
=-\end{array}$ & $\overline{0}$ & - \\
\hline $\begin{array}{l}w \\
w \\
w \\
w \\
w\end{array}$ & $\begin{array}{l}201 \\
203 \\
204 \\
205 \\
206\end{array}$ & $\begin{array}{l}413726 N 0710416.1 \\
413356 N 0710531.1 \\
413028 N 0710637.1 \\
413015 N 0710628.1 \\
413024 N 0710554.1\end{array}$ & $\begin{array}{r}119 \\
170 \\
44 \\
11 \\
35\end{array}$ & $\begin{array}{l}\text { DIAS,RAYMOND } \\
\text { VINCENT O J J } \\
\text { ACOAXET WAT.CO. } \\
\text { ACOAXET WAT.CO. } \\
\text { WESTPORT AQUED. }\end{array}$ & $\begin{array}{c}1972 \\
1972 \\
-- \\
1906\end{array}$ & $\begin{array}{l}P \\
P \\
C \\
D \\
C\end{array}$ & $\begin{array}{r}6 \\
6 \\
-5 \\
104 \\
6\end{array}$ & $\begin{array}{l}x \\
x \\
x \\
x\end{array}$ & $\begin{array}{r}110 \\
170 \\
130 \\
16 \\
86\end{array}$ & $\begin{array}{l}w \\
w \\
w \\
w\end{array}$ & $\begin{array}{l}35 \\
=- \\
=-\end{array}$ & $\begin{array}{l}=- \\
=- \\
=-\end{array}$ & $\begin{array}{l}=- \\
=- \\
35\end{array}$ & $\begin{array}{l}= \\
= \\
=- \\
-06\end{array}$ & $\begin{array}{l}H \\
H \\
P \\
P \\
P\end{array}$ & $\begin{array}{r}3 \\
5 \\
5 \\
12 \\
20\end{array}$ & $\begin{array}{l}=- \\
=- \\
=-\end{array}$ & $\begin{array}{l}=- \\
=- \\
=-\end{array}$ & - & $\begin{array}{l}\bar{M} \\
M \\
M\end{array}$ \\
\hline$x$ & $\begin{array}{r}207 \\
2\end{array}$ & $\begin{array}{l}413024 N 0710554.2 \\
413021 N 0710318.1\end{array}$ & $\begin{array}{l}35 \\
29\end{array}$ & $\begin{array}{l}\text { WESTPORT AQUEO. } \\
\text { HORSENECK BEACH }\end{array}$ & $\begin{array}{l}1906 \\
1956\end{array}$ & $w$ & $\begin{array}{l}6 \\
1\end{array}$ & $\begin{array}{l}x \\
0\end{array}$ & 95 & $\mathbf{W}$ & 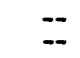 & 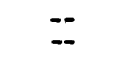 & $\begin{array}{l}35 \\
19\end{array}$ & $\begin{array}{r}-06 \\
7-56\end{array}$ & $\begin{array}{l}P \\
U\end{array}$ & $\begin{array}{l}30 \\
--\end{array}$ & $\ddot{-\infty}$ & $=$ & $\overrightarrow{\mathrm{D}}$ & - \\
\hline
\end{tabular}


Table 2.--Logs of selected wells, test wells, and borings

(Depths are given in feet below land surface)

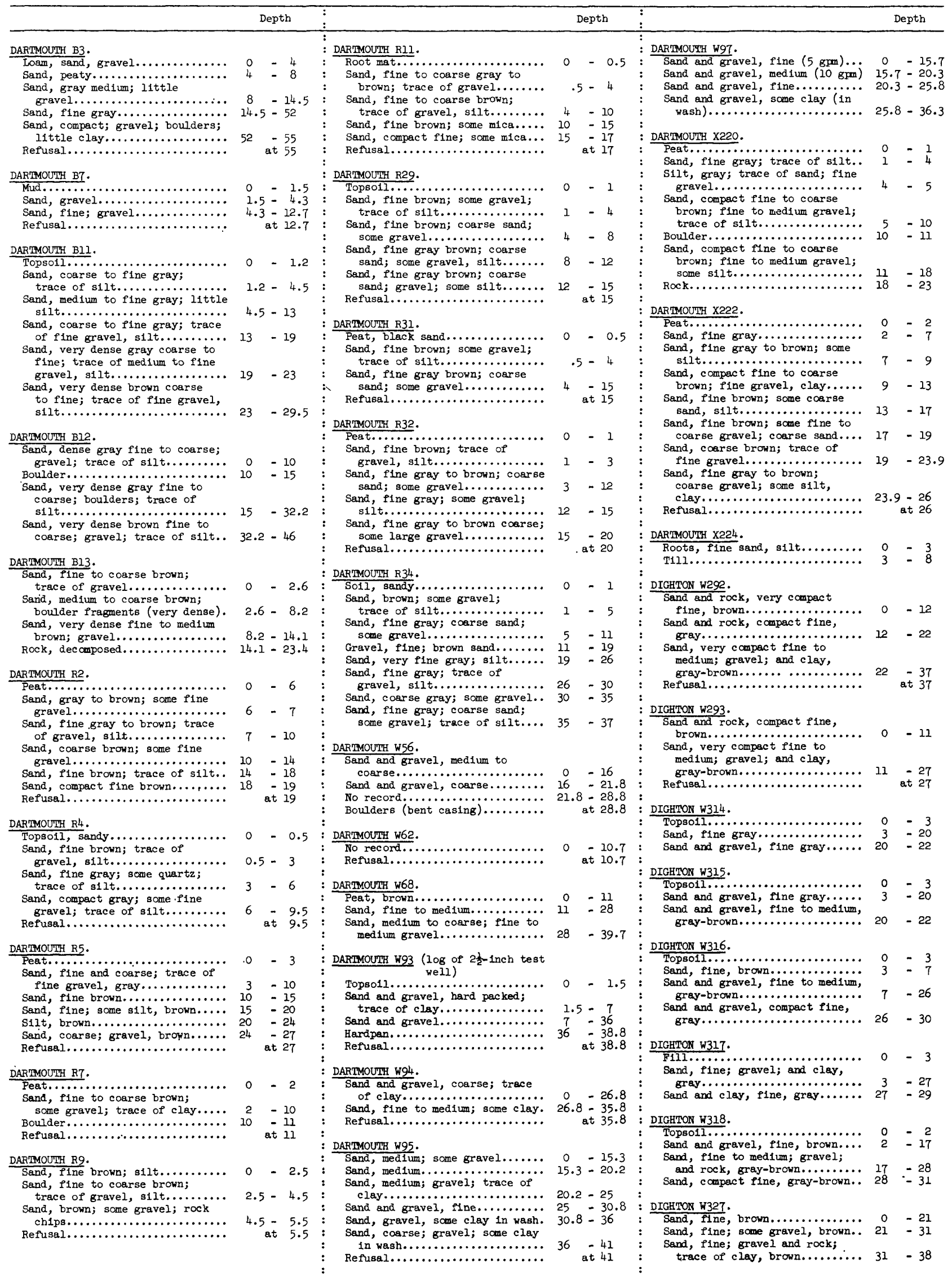


Table 2.--Logs of selected wells, test wells, and borings (Continued)

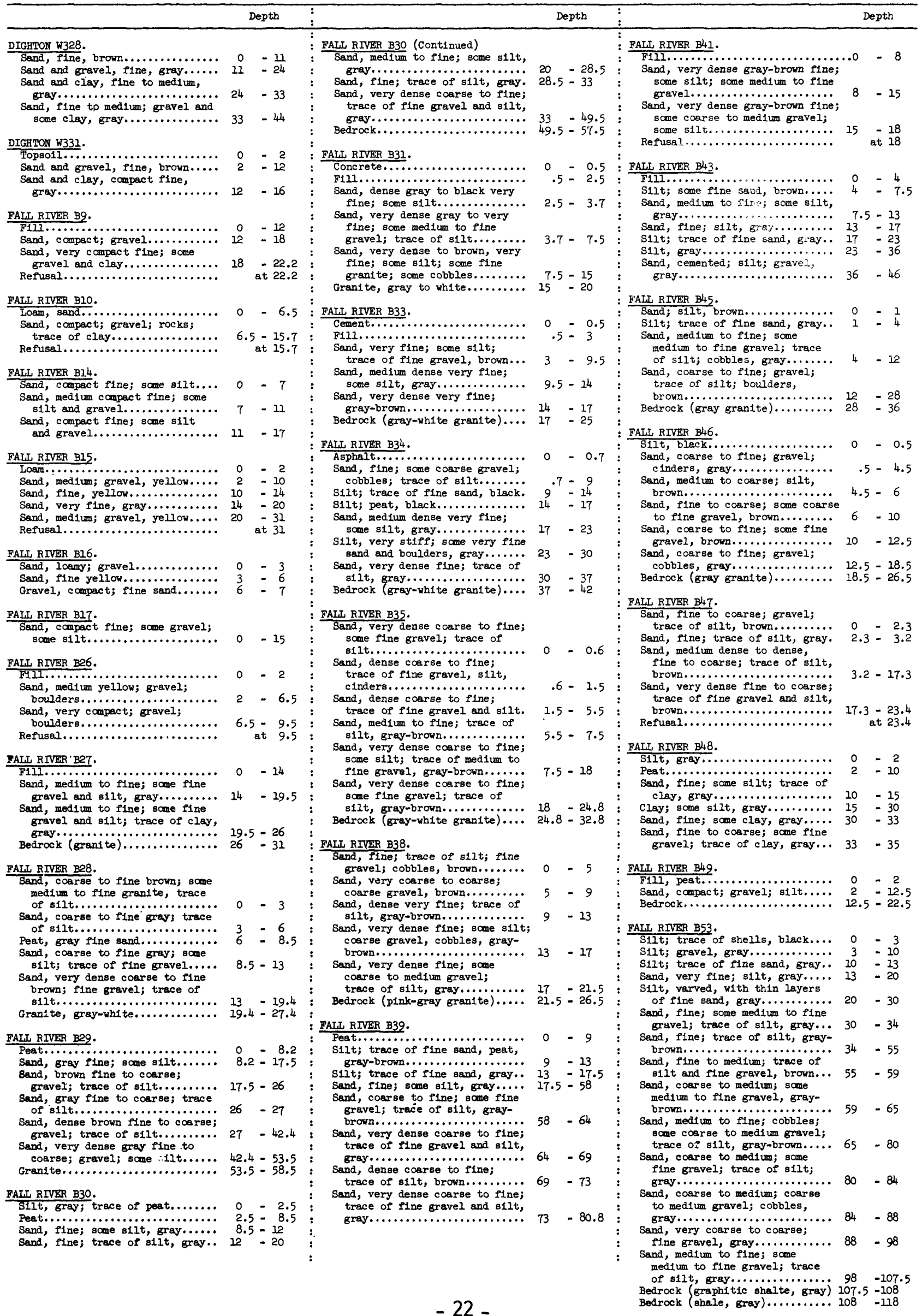


Table 2.--Logs of selected wells, test wells, and borings (Continued)

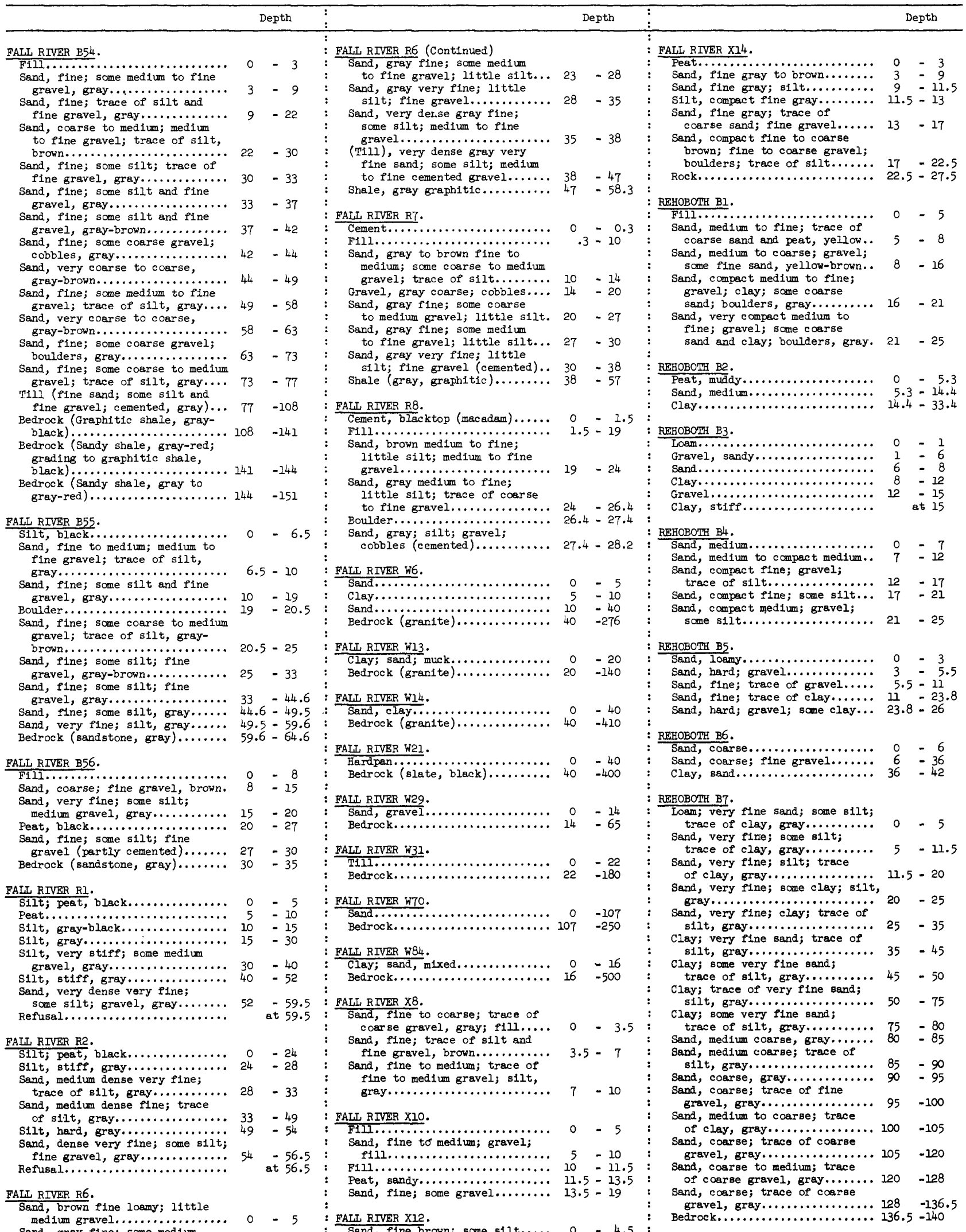

Sand, gray fine; some medium

aral; trace of silt; cobbles. $5-8$

gravel; trace of silt; cobbles.

and, gray fline; some medium to $8-13$

fine gravel; cobbles............

fine gravel; trace of silt;

boulders.................... $13-20$

Sand, gray very fine; ittle

silt; medium to fine gravel... $20 \quad-23$

nd, gray fine; some medium

silt; fine gravel............

gine silt; medium to fin

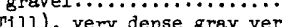

fine sand; some silt; medil

to fine cemented gravel....... $38-47$

ALL RIVER RT.

$0-0.3$

to

medium; some coarse to medium

gravel; trace of silt........ 10 - I4

to medium gravel; little silt. $20-27$

to fine gravel; little silt... $27 \quad-30$

nd, gray very fine; little

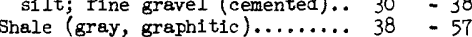

ALL RIVER R8.

nt, blacktop (macadam)

and, brown medium to fine;

little silt; medium to fine

and, gray medium to fine;

little silt; trace of coerse

to fine gravel.............. $24 \quad-26.4$

(1).

(con. 27.2

SLL RTVER W6

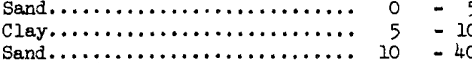

Bedrock (granite)............. 40 , -276

CLIL RIVER W13.

Bedrock (aranite)............ $20 \quad-140$

ALI RIVER W14

LLI RIVER W2I.

Bedrock (slate, black) ........ $40-400$

ALI RIVER W29.

Bedrock................... $14=65$

FALL RIVER W3I

SAL RIVER WTO.

LIL RIVER W84.

Clay; sand, mlxed.............. 00 - 16

FALL R IVER X8.

and, fline to coarse; trace of

coarse gravel, gray; flll.

flne grovel, brown...........

and, fine to medium; trace of

fine to medium gravel; silt,

LI RIVER X10

Sand, fine to medium; gravel;

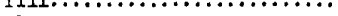

Peat, sandy.................. $11.5=13.5$

FALL RIVER X12.

Sand, come brown; some silt.

some silt. very flne gray;

sand, compect fine brown; some

fine gravel; trace of silt....

Gravel, very compact fine; rock

fragments....................

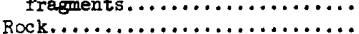

$0-4.5$

$4.5-5.5$

$5.5-6.5$

$6.5-8$
LLL RTVER X14

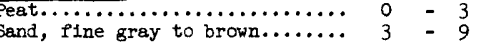

Sand, fine gray; silt.......... 9 . $\begin{aligned} & 91 . \\ & \text { Silt, compact fine gray........ } 11.5=13\end{aligned}$

nd, fine gray; trace of

nd, compact fine to coarse

brown; fine to coarse gravel

$17-22.5$

HOBOTH BI.

and, medium to fine; trace of

, medium to coarse; gravel; $8-16$

and, compact medium to fine,

d, very compact medium to

sand and clay; boulders, gray. $21-25$

$\mathrm{OBOTH} \mathrm{B2}$

Peat, muddy................. 0.5 .3

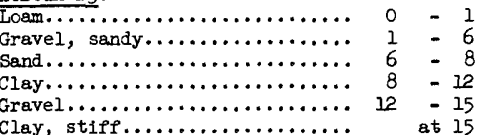

HOBOTH B4.

Sand, medium............... $0=7$ nd, compact flne; gravel;

trace of silt.

Sand, compact flne; some silt... 17 - 21

some silt................. $21-25$

Sand, hard; gravel............ $3=3.5$

Sand, fine; trace of clay...... 11 - 23.8

HOBOTH B6.

Sand, coarse; fine gravel........ $6=6$

BOBOTH B7.

oam; very flne sand; some silt;

$0-5$

trece of clay, groy.

Sand, very flne; silt; trace

gray............. 20 - 25

, very plne; clay, trace of

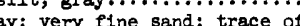

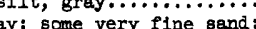

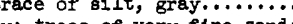

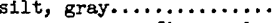

$80-85$

d, coarse, gray.......... 90 - 95

gravel, gray................ $95 \quad-100$

Sand, medium to coarse; trace $100-105$

and, coarse; trace of coarse

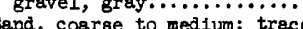

of carse gravel, gray.........

Bedrock....................... 136.5 - 140 
Table 2.--Iogs of selected wells, test wells, and borings (Continued)

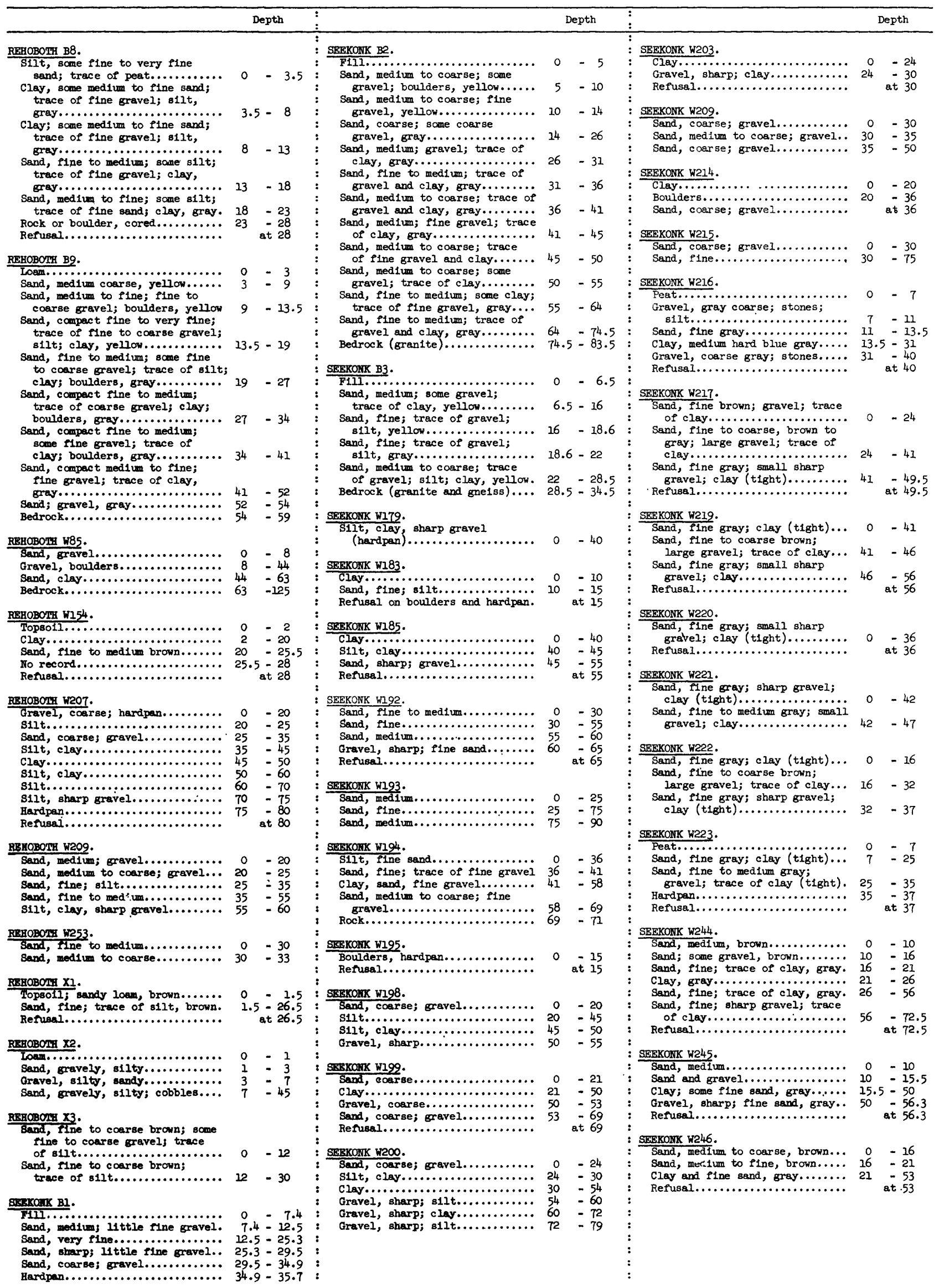


Table 2.--Logs of selected wells, test wells, and borings (Cont1nued)

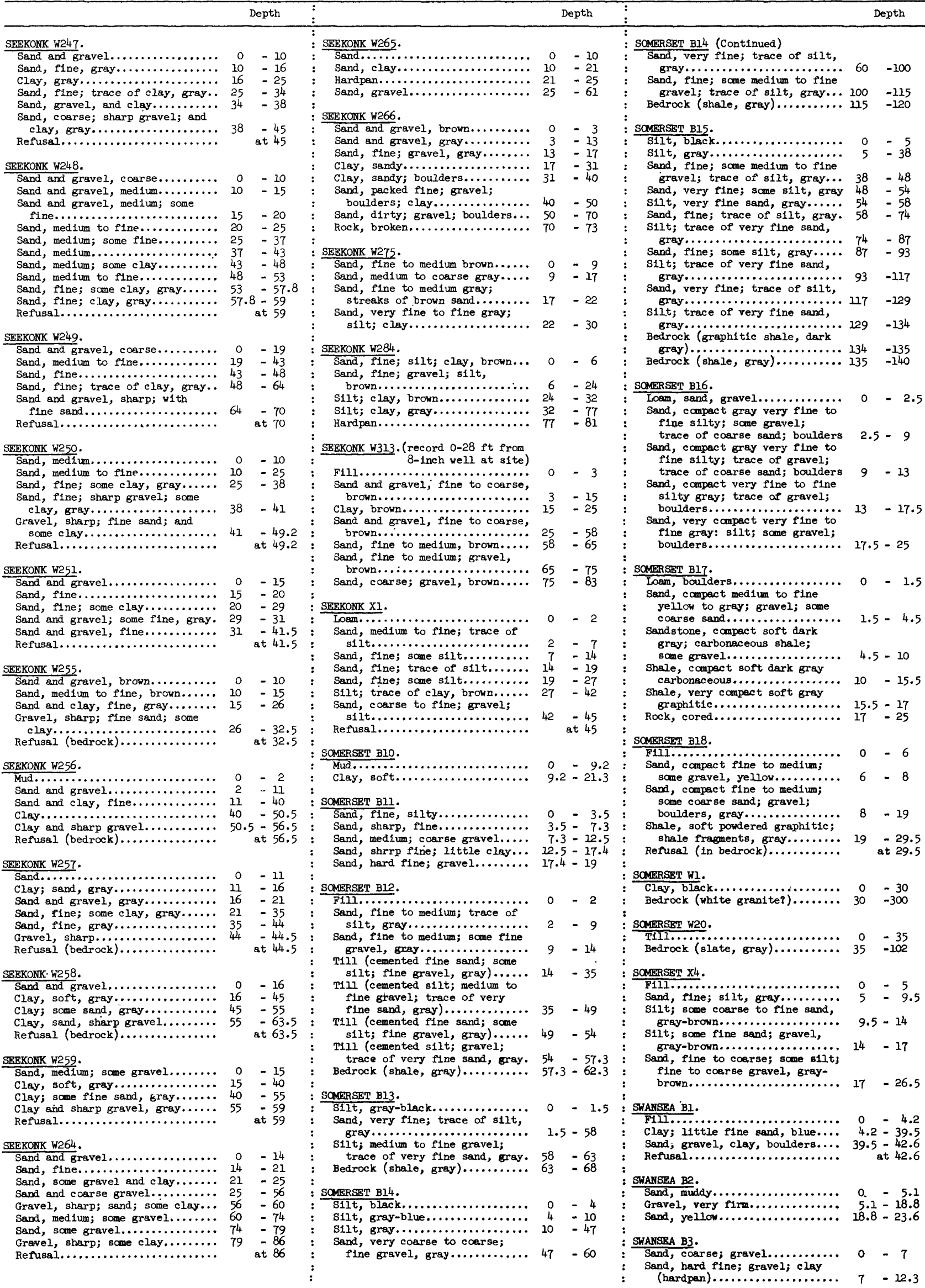


Trable 2.--Logs of selected wells, test wells, and borlngs (Continued)

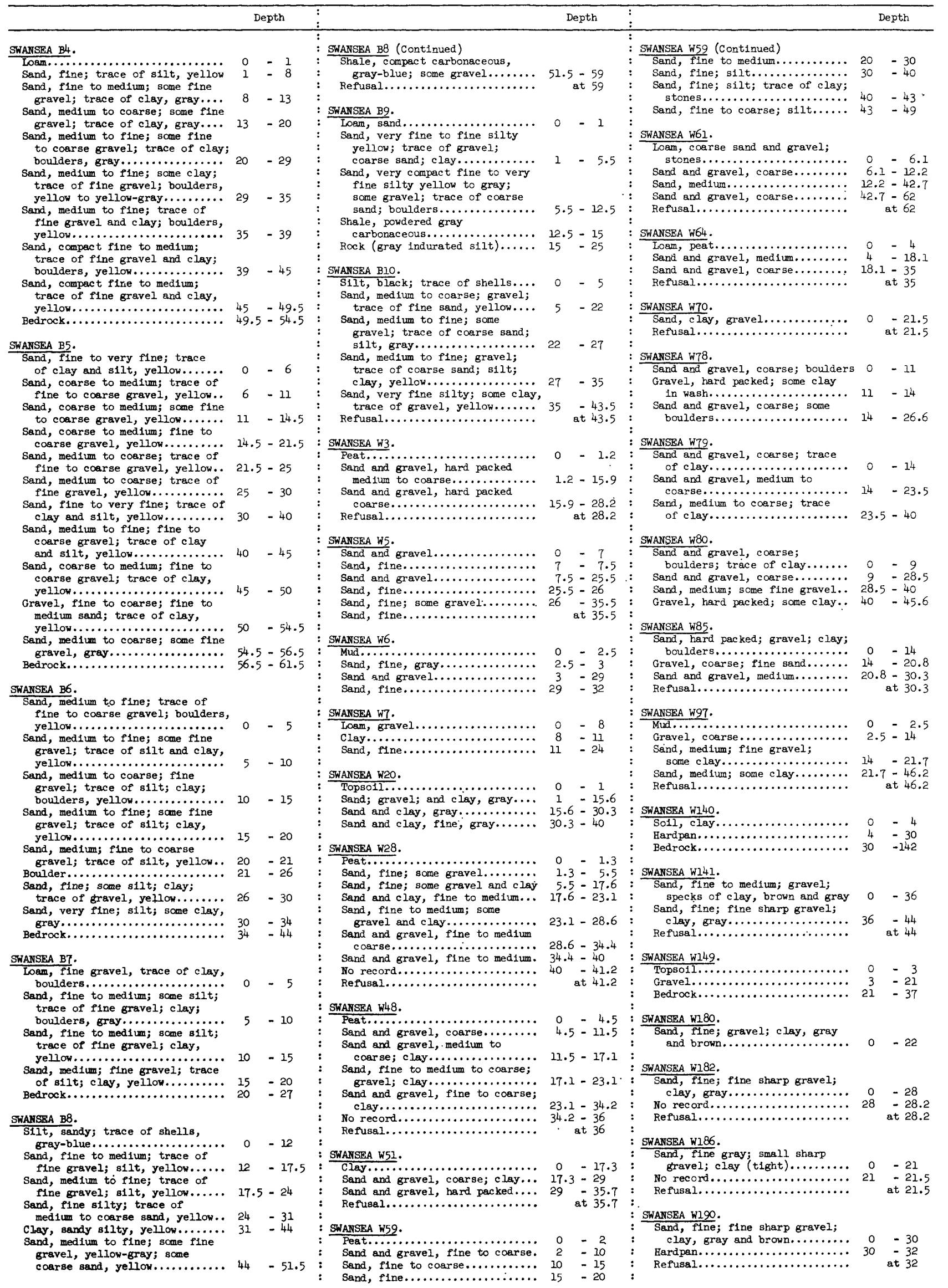


Table 2.--Logs of selected wells, test wells, and borings (Continued)

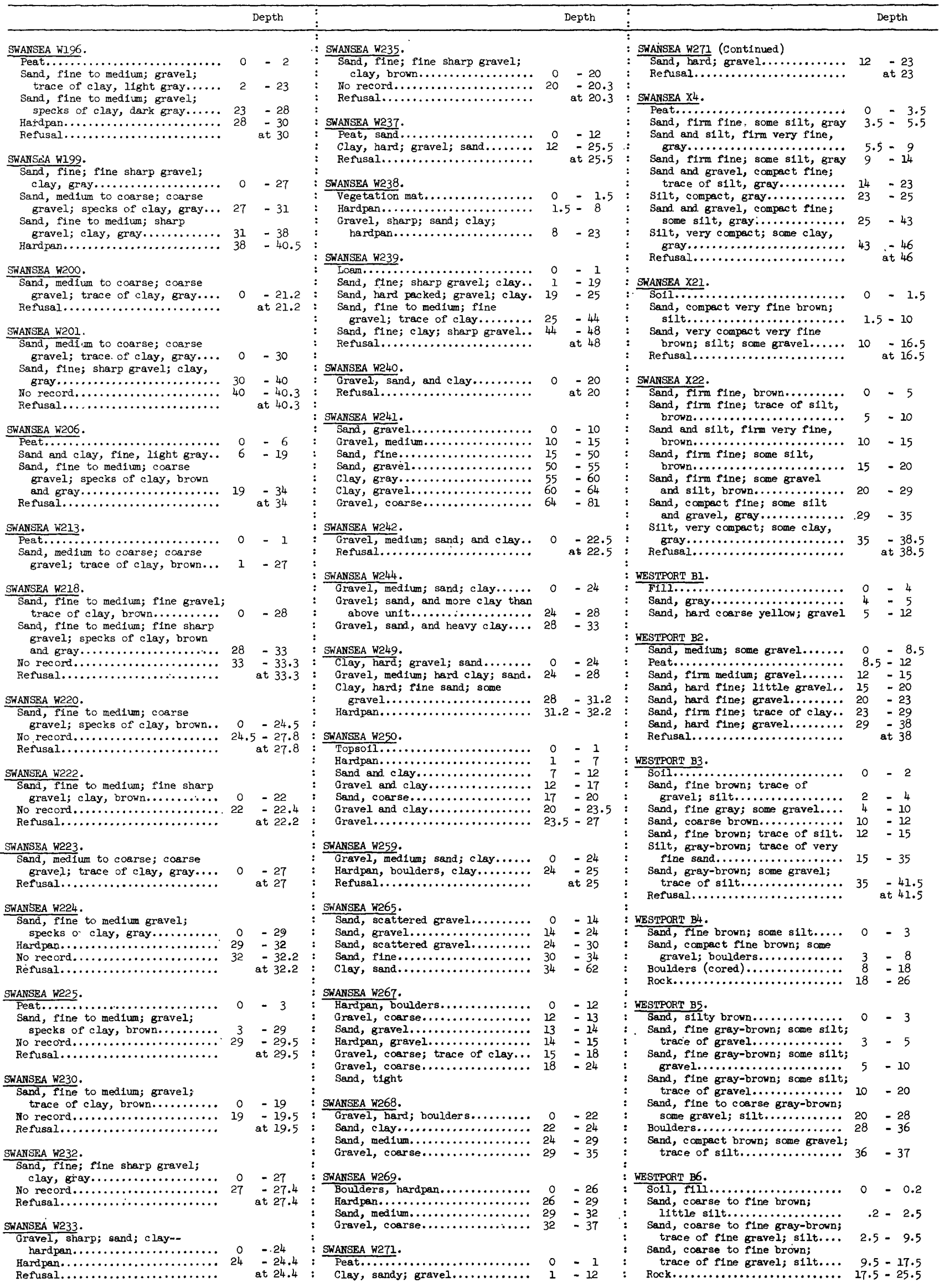


Table 2.--Logs of selected wells, test wells, and borings (Continued)

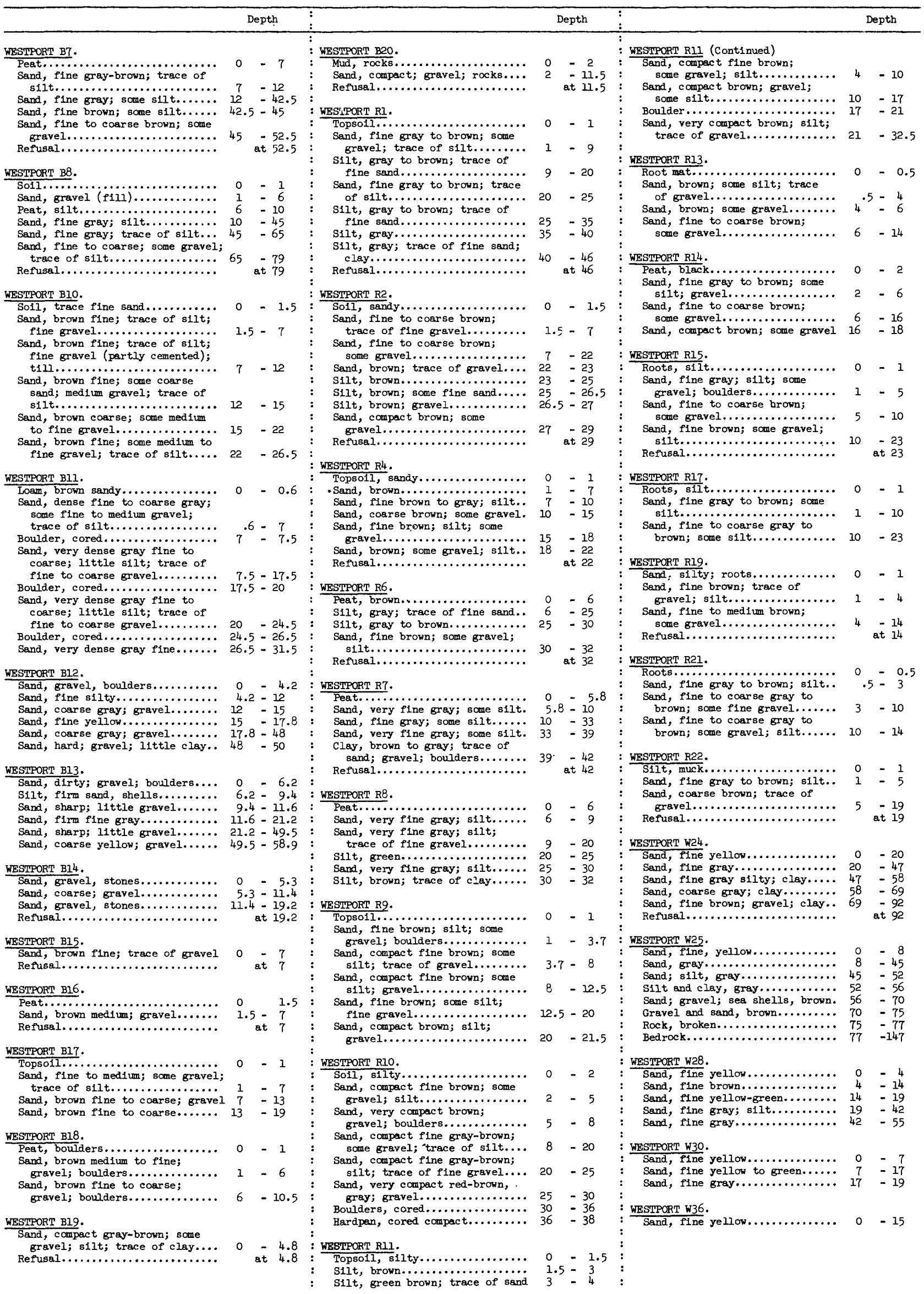


Table 2.--Logs of selected wells, test wells, and borings (Continued)

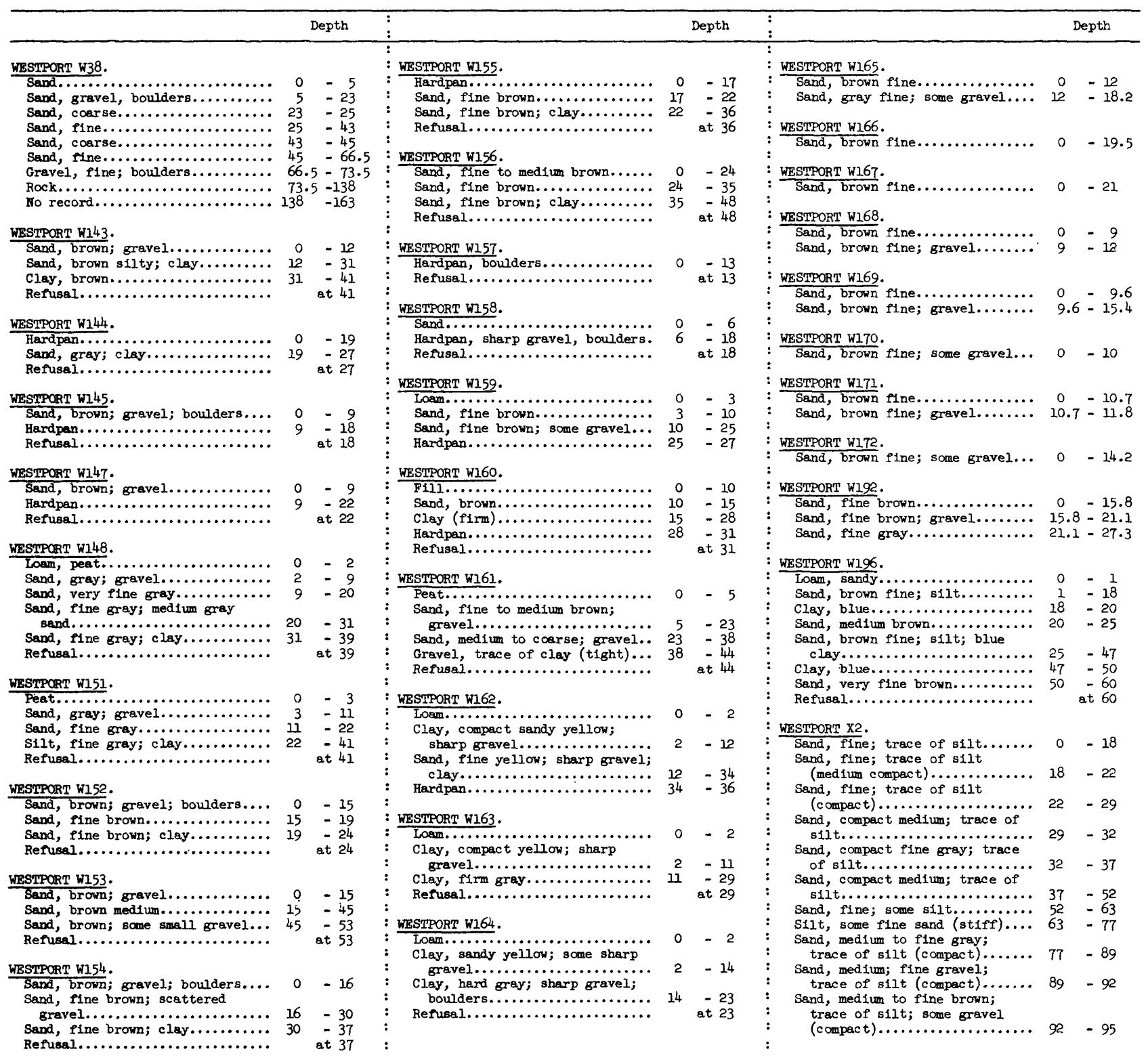


Table 3.--Chemical analyses of ground water

(Source of data: 1. U.S. Geological Survey; 3, State Hea1th Department; 6, Private; and 8, other.)

\begin{tabular}{|c|c|c|c|c|c|c|c|c|c|c|c|c|c|c|c|c|c|c|}
\hline $\begin{array}{c}\text { Local } \\
\text { we } 11 \\
\text { number }\end{array}$ & $\begin{array}{c}\text { Date } \\
\text { of } \\
\text { sample }\end{array}$ & Col or & $\mathrm{pH}$ & $\begin{array}{c}\text { Alka- } \\
1 \text { inity } \\
\text { as } \\
\mathrm{CaCO}_{3} \\
(\mathrm{mg} / \mathrm{L})\end{array}$ & $\begin{array}{l}\text { Hard- } \\
\text { ness } \\
(\mathrm{mg} / \mathrm{L})\end{array}$ & $\begin{array}{l}\mathrm{Ca} 1- \\
\text { ci un } \\
(\mathrm{mg} / \mathrm{L})\end{array}$ & $\begin{array}{l}\text { Magne- } \\
\text { sium } \\
(\mathrm{mg} / \mathrm{L})\end{array}$ & $\begin{array}{l}\text { Sodium } \\
(\mathrm{mg} / \mathrm{L})\end{array}$ & $\begin{array}{l}\text { Potas- } \\
\text { sium } \\
(\mathrm{mg} / \mathrm{L})\end{array}$ & $\begin{array}{l}\text { Ir on } \\
(\mathrm{mg} / \mathrm{L})\end{array}$ & $\begin{array}{l}\text { Man- } \\
\text { ganese } \\
(\mathrm{mg} / \mathrm{L})\end{array}$ & $\begin{array}{l}\text { Silica } \\
\text { (mg/L) }\end{array}$ & $\begin{array}{l}\text { Sul- } \\
\text { fate } \\
(\mathrm{mg} / \mathrm{L})\end{array}$ & $\begin{array}{l}\text { Chlo- } \\
\text { ride } \\
(\mathrm{mg} / \mathrm{L})\end{array}$ & $\begin{array}{c}\text { Specific } \\
\text { conduct- } \\
\text { ance } \\
\text { (micro- } \\
\text { mhos) }\end{array}$ & $\begin{array}{l}\mathrm{Ni}- \\
\text { trate } \\
(\mathrm{mg} / \mathrm{L})\end{array}$ & $\begin{array}{l}\text { Copper } \\
(\mathrm{mg} / \mathrm{L})\end{array}$ & $\begin{array}{c}\text { Source } \\
\text { of } \\
\text { data }\end{array}$ \\
\hline
\end{tabular}

DARTMOUTH
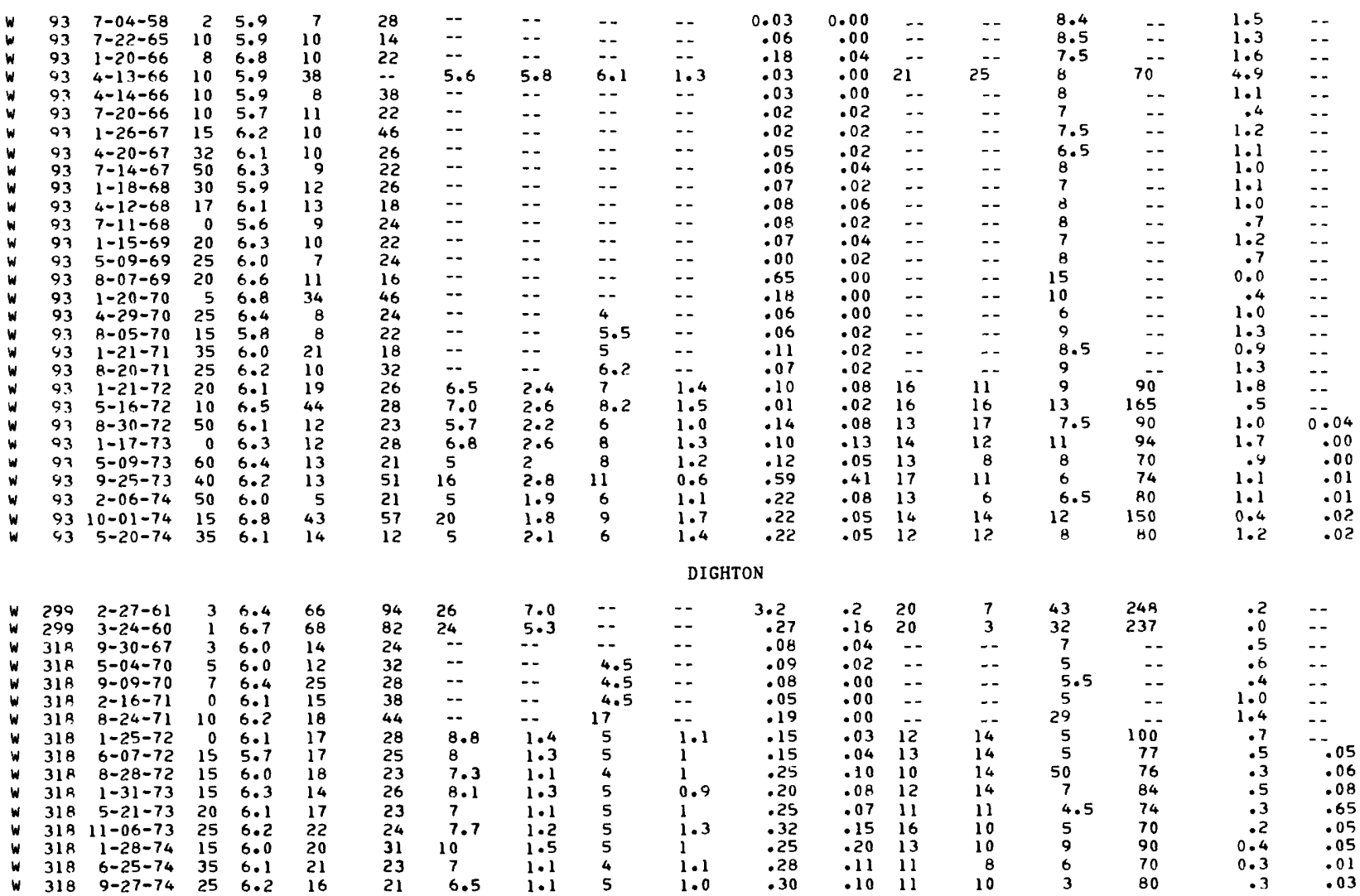

DIGHTON
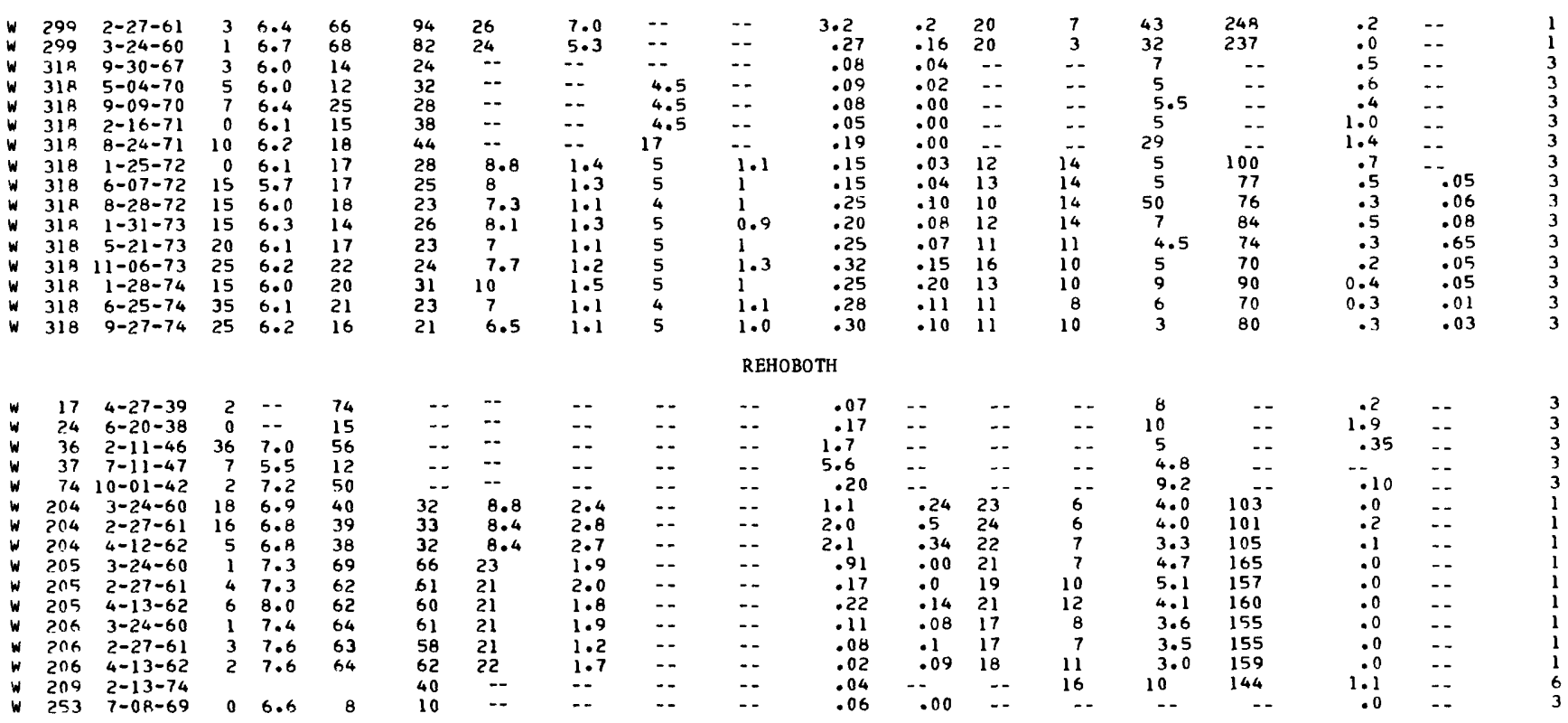

REHOBOTH
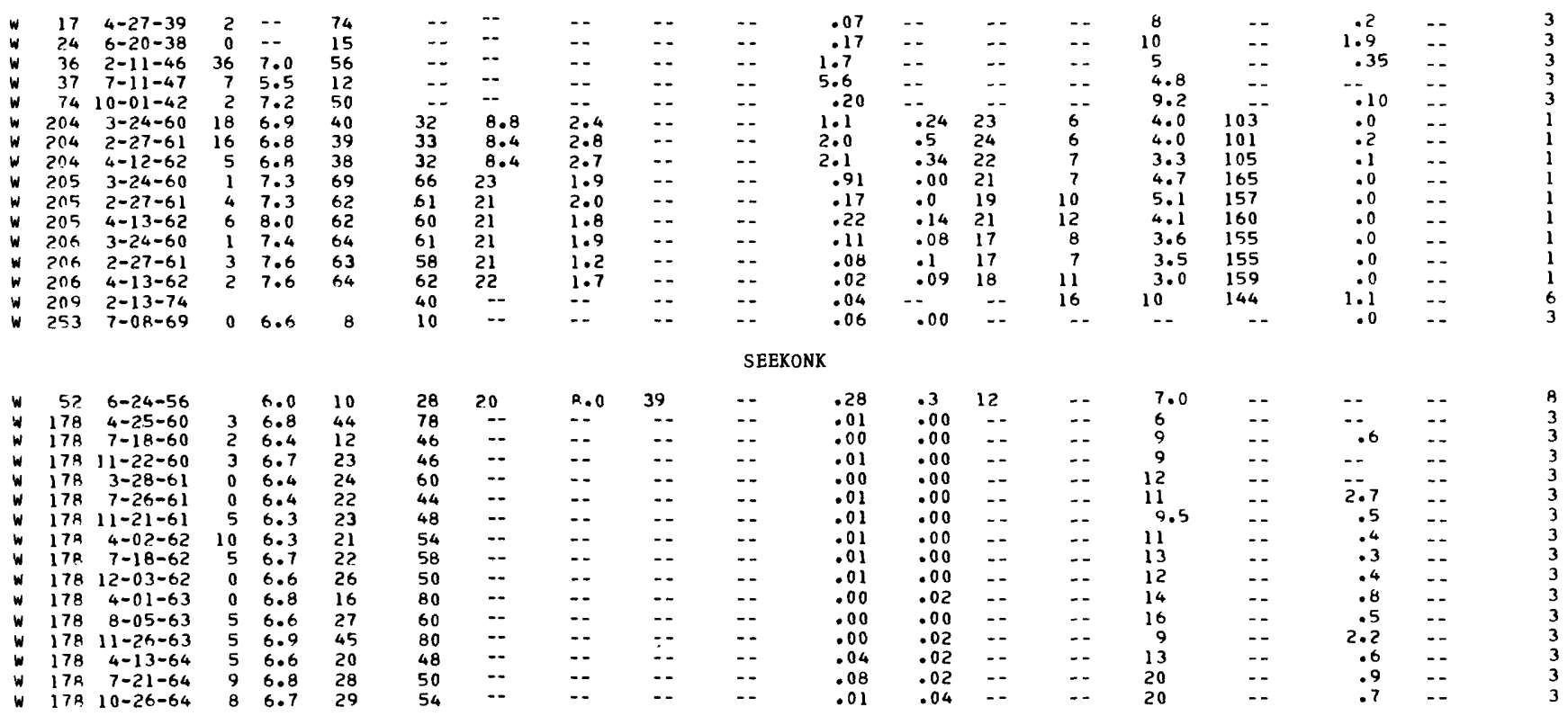

SEEKONK 
Table 3.--Chemical analyses of ground water (continued)

\begin{tabular}{|c|c|c|c|c|c|c|c|c|c|c|c|c|c|c|c|c|c|}
\hline $\begin{array}{c}\text { L } \propto \text { a } 1 \\
\text { we } 11 \\
\text { number }\end{array}$ & $\begin{array}{c}\text { Date } \\
\text { of } \\
\text { sample }\end{array}$ & Col or $\mathrm{pH}$ & $\begin{array}{c}\text { Alka- } \\
\text { linity } \\
\text { as } \\
\mathrm{CaCO} \\
(\mathrm{mg} / \mathrm{L})\end{array}$ & $\begin{array}{l}\text { Hard - } \\
\text { ness } \\
(\mathrm{mg} / \mathrm{L})\end{array}$ & $\begin{array}{l}\mathrm{Ca} 1 \text { - } \\
\mathrm{Cium} \\
(\mathrm{mg} / \mathrm{L})\end{array}$ & $\begin{array}{l}\text { Magne - } \\
\text { sium } \\
(\mathrm{mg} / \mathrm{L})\end{array}$ & $\begin{array}{l}\text { Sodiun } \\
(\mathrm{mg} / \mathrm{L})\end{array}$ & $\begin{array}{l}\text { Potas - } \\
\text { siun } \\
\text { (mg L) }\end{array}$ & $\begin{array}{c}\text { Ir on } \\
(\mathrm{ng} / \mathrm{L})\end{array}$ & $\begin{array}{c}\text { Man- } \\
\text { ganese } \\
(\mathrm{mg} / \mathrm{L})\end{array}$ & $\begin{array}{l}\text { Silica } \\
(\mathrm{ng} / \mathrm{L})\end{array}$ & $\begin{array}{l}\text { Sul - } \\
\text { fate } \\
(\mathrm{mg} / \mathrm{L})\end{array}$ & $\begin{array}{l}\text { Chlo- } \\
\text { ride } \\
(\mathrm{mg} / \mathrm{L})\end{array}$ & $\begin{array}{l}\text { Specific } \\
\text { conduct- } \\
\text { ance } \\
\text { (micro- } \\
\text { mhos) }\end{array}$ & $\begin{array}{l}\mathrm{Ni}- \\
\text { trate } \\
(\mathrm{mg} / \mathrm{L})\end{array}$ & $\begin{array}{l}\text { Copper } \\
(\mathrm{mg} / \mathrm{L})\end{array}$ & $\begin{array}{c}\text { Source } \\
\text { of } \\
\text { data }\end{array}$ \\
\hline
\end{tabular}

\begin{tabular}{|c|c|c|c|c|c|c|c|c|c|c|c|}
\hline & & & & & & & & & & CONK ( & ont i nue \\
\hline$w$ & 178 & $2-17-65$ & 3 & 6.5 & 28 & 54 & -- & -- & -- & -- & 0.02 \\
\hline$w$ & 178 & $5-18-65$ & 3 & 6.5 & 25 & 54 & -- & -- & $\cdots$ & -- & .03 \\
\hline$w$ & 178 & $8-16-65$ & 0 & 6.9 & 41 & 76 & - & -- & -- & $=-$ & .01 \\
\hline$w$ & 178 & $2-21-66$ & 10 & 6.9 & 42 & 82 & -- & $\cdots$ & -- & $\cdots$ & .25 \\
\hline$w$ & 178 & $6-0 R-66$ & 5 & 6.4 & 23 & 54 & -- & -- & -- & -- & .01 \\
\hline$w$ & 178 & $8-22-66$ & 2 & 7.0 & 47 & 88 & -- & -- & - & -- & .03 \\
\hline$w$ & 178 & $2-20-67$ & 2 & $6 \cdot 5$ & 33 & 58 & -- & - & - & -- & .07 \\
\hline$w$ & 178 & $5-22-67$ & 0 & 7.1 & 25 & 48 & -- & -- & $\cdots$ & -- & .07 \\
\hline$w$ & 178 & $8-21-67$ & 5 & 6.6 & 27 & 64 & -- & -- & -- & -- & .03 \\
\hline$w$ & 178 & $2-21-68$ & 0 & 6.8 & 32 & 48 & -- & -- & -- & -- & .02 \\
\hline$w$ & $\begin{array}{l}178 \\
178\end{array}$ & $\begin{array}{l}5-21-68 \\
8-19-68\end{array}$ & $\begin{array}{l}2 \\
5\end{array}$ & 7.1 & 33 & 84 & $\because-$ & $\because$ & - & $\cdots$ & .03 \\
\hline$w$ & & & $\begin{array}{l}5 \\
5\end{array}$ & $\begin{array}{l}6.5 \\
6.8\end{array}$ & $\begin{array}{l}42 \\
44\end{array}$ & $\begin{array}{l}50 \\
86\end{array}$ & -- & -- & $=$ & -- & .00 \\
\hline$w$ & $\begin{array}{l}178 \\
178\end{array}$ & $\begin{array}{l}3-04-69 \\
6-24-69\end{array}$ & $\begin{array}{l}5 \\
5\end{array}$ & $\begin{array}{l}6.8 \\
6.5\end{array}$ & $\begin{array}{l}44 \\
28\end{array}$ & $\begin{array}{l}86 \\
80\end{array}$ & -- & -- & -- & -- & .01 \\
\hline$w$ & & $9-10-69$ & & $\begin{array}{l}6.5 \\
7.1\end{array}$ & & $\begin{array}{l}80 \\
86\end{array}$ & 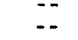 & - & - & -- & .02 \\
\hline$w$ & $\begin{array}{l}178 \\
178\end{array}$ & $3-03-70$ & $\begin{array}{l}0 \\
5\end{array}$ & $\begin{array}{l}7.1 \\
6.8\end{array}$ & $\begin{array}{l}44 \\
29\end{array}$ & $\begin{array}{l}86 \\
52\end{array}$ & - & -- & -. & -- & .04 \\
\hline$w$ & $\begin{array}{l}178 \\
178\end{array}$ & $6-16-70$ & $\begin{array}{l}5 \\
0\end{array}$ & $\begin{array}{l}6.8 \\
6.5\end{array}$ & 23 & 50 & - & -- & 12 & -- & .02 \\
\hline$w$ & $\begin{array}{l}178 \\
178\end{array}$ & $9-22-70$ & 0 & $\begin{array}{l}6.5 \\
6.9\end{array}$ & 52 & 84 & - - & -- & 7 & -- & .03 \\
\hline w & & $3-15-71$ & 1 & 6.7 & 46 & 86 & $\ldots$ & -- & -- & & .05 \\
\hline$w$ & $\begin{array}{l}178 \\
178\end{array}$ & $7-12-71$ & 0 & 7.0 & $\begin{array}{l}40 \\
54\end{array}$ & 80 & $\ldots$ & $\ldots$ & 9 & $\because-$ & .00 \\
\hline$w$ & $\begin{array}{l}178 \\
178\end{array}$ & $\begin{array}{l}7-12-11 \\
3-21-72\end{array}$ & 0 & 7.0 & $\begin{array}{l}54 \\
52\end{array}$ & 85 & 28 & 3.6 & 10 & 1.0 & .00 \\
\hline$w$ & $\begin{array}{l}178 \\
178\end{array}$ & $7-17-72$ & 0 & 6.8 & $\begin{array}{l}52 \\
58\end{array}$ & $\begin{array}{l}83 \\
82\end{array}$ & 27 & 3.6 & 11 & $\begin{array}{l}1.0 \\
1.2\end{array}$ & .00 \\
\hline$w$ & 178 & $10-16-72$ & 5 & 6.7 & 55 & 80 & & & & 1.2 & .02 \\
\hline$w$ & 178 & $\begin{array}{r}10-16-72 \\
3-19-73\end{array}$ & 0 & 6.8 & $\begin{array}{l}53 \\
62\end{array}$ & $\begin{array}{l}80 \\
94\end{array}$ & 26 & 3.7 & 10 & 1.1 & .01 \\
\hline$w$ & 178 & & $\begin{array}{l}0 \\
5\end{array}$ & $\begin{array}{l}6.8 \\
6.8\end{array}$ & $\begin{array}{l}62 \\
45\end{array}$ & $\begin{array}{l}94 \\
81\end{array}$ & 32 & 3.5 & 9 & $1 \cdot 1$ & .00 \\
\hline$w$ & 178 & $\begin{array}{r}11-13-73 \\
3-25-74\end{array}$ & $\begin{array}{l}5 \\
0\end{array}$ & $\begin{array}{l}6.8 \\
6.9\end{array}$ & $\begin{array}{l}45 \\
53\end{array}$ & $\begin{array}{l}81 \\
71\end{array}$ & 27 & 3.3 & 10 & 1.1 & .00 \\
\hline$w$ & 178 & $3-25-74$ & 0 & 6.9 & 53 & 71 & 23 & 3.4 & 10 & 1 & .00 \\
\hline$w$ & 178 & $7-23-74$ & 0 & 6.9 & 62 & 77 & 25 & 3.6 & 13 & 1 & .00 \\
\hline$w$ & 178 & $11-18-74$ & 0 & $7 \cdot 1$ & 51 & 79 & 26 & 3.3 & 9 & 1.2 & .00 \\
\hline$w$ & 194 & $6-10-74$ & 5 & 6.5 & 37 & 51 & 16 & 2.7 & 9 & 1 & .02 \\
\hline$w$ & 199 & $8-10-73$ & -- & -- & $\because$ & - & $-\cdot$ & -- & -- & -- & .44 \\
\hline$w$ & 209 & $2-26-74$ & -- & -- & $\because$ & -- & $\cdots$ & - & $\because$ & - & .10 \\
\hline$w$ & 214 & $6-10-74$ & 25 & 6.5 & $\begin{array}{r}40 \\
590\end{array}$ & $\begin{array}{r}54 \\
374\end{array}$ & 100 & 2.8 & 10 & .5 & .70 \\
\hline$w$ & $\begin{array}{l}215 \\
265\end{array}$ & $\begin{array}{l}6-10-74 \\
4-25-60\end{array}$ & $\begin{array}{r}350 \\
3\end{array}$ & $\begin{array}{l}6.6 \\
6.5\end{array}$ & $\begin{array}{r}590 \\
19\end{array}$ & $\begin{array}{r}374 \\
44\end{array}$ & 100 & 30 & 100 & 3.0 & ${ }^{70} .01$ \\
\hline$w$ & 265 & $7-18-60$ & 3 & 6.5 & 26 & 58 & -- & -- & -- & -- & $\begin{array}{l}.01 \\
.00\end{array}$ \\
\hline$w$ & 265 & $11-22-60$ & 3 & 6.6 & 28 & 54 & -- & -- & $\cdots$ & -- & .01 \\
\hline$w$ & 265 & $3-28-61$ & 5 & 6.4 & 22 & 46 & -- & -- & $\cdots$ & - & .00 \\
\hline$w$ & 265 & $7-26-61$ & 0 & 6.4 & 21 & 44 & -- & -- & -- & - & .01 \\
\hline$w$ & 265 & $11-21-61$ & 5 & 6.3 & 22 & 48 & -- & -- & $\cdots$ & $\cdots$ & .01 \\
\hline$w$ & 265 & $4-02-62$ & 10 & 6.2 & 22 & 54 & -- & - & -- & -- & .01 \\
\hline$w$ & 265 & $7-18-62$ & 5 & $7 \cdot 5$ & 38 & 74 & -- & $\cdots$ & $\cdots$ & $\cdots$ & .01 \\
\hline$w$ & 265 & $12-03-62$ & 0 & 6.8 & 25 & 50 & -- & -- & -- & $\cdots$ & .01 \\
\hline$w$ & 265 & $4-01-63$ & 0 & 6.6 & 23 & 70 & -- & -- & -- & -- & .06 \\
\hline$w$ & 265 & $8-05-63$ & 5 & 6.5 & 26 & 60 & -- & $\cdots$ & -- & - & .00 \\
\hline$w$ & 265 & $11-26-63$ & 5 & 6.8 & 32 & 60 & $\cdots$ & -- & -- & $\cdots$ & .00 \\
\hline$w$ & 265 & $4-13-64$ & 5 & 6.6 & 20 & 48 & $\cdots$ & $\cdots$ & -- & $\cdots$ & .20 \\
\hline$w$ & 265 & $7-21-64$ & 11 & 6.9 & 33 & 60 & -- & $\because$ & -- & -- & .02 \\
\hline$w$ & 265 & $10-26-64$ & 10 & 6.7 & 29 & 56 & -- & -- & -- & -- & .01 \\
\hline$w$ & 265 & $2-17-65$ & 3 & 6.5 & 27 & 58 & -- & -- & - & -- & .01 \\
\hline$w$ & 265 & $5-18-65$ & 3 & 6.6 & 25 & 54 & -- & -- & -- & -- & .02 \\
\hline$w$ & 265 & $8-16-65$ & 5 & 6.4 & 24 & 56 & -- & -- & $\cdots$ & -- & .02 \\
\hline$w$ & 265 & $2-21-66$ & 5 & 6.6 & 29 & 66 & -- & -- & $\cdots$ & -- & .01 \\
\hline$w$ & 265 & $6-08-66$ & 5 & 6.5 & 22 & 54 & -- & -- & -- & - & .01 \\
\hline$w$ & 265 & $8-22-66$ & 5 & 6.6 & 35 & 62 & -- & $\cdots$ & -- & -- & .08 \\
\hline$w$ & 265 & $2-20-67$ & 2 & 6.7 & 40 & 72 & - & $\cdots$ & $\cdots$ & -- & .02 \\
\hline$w$ & 265 & $5-22-67$ & 3 & $8 \cdot 3$ & 51 & 76 & -- & -- & - & -- & .03 \\
\hline$w$ & 265 & $8-21-67$ & 5 & 6.6 & 28 & 70 & -- & -- & $-\cdot$ & -- & .02 \\
\hline$w$ & 265 & $2-21-68$ & 0 & 7.0 & 39 & 58 & -- & -- & -- & -- & .12 \\
\hline$w$ & 265 & $5-21-68$ & 2 & 7.1 & 35 & 54 & -- & $\cdots$ & $\cdots$ & -- & .01 \\
\hline$w$ & 265 & $8-19-68$ & 5 & 6.6 & 48 & 60 & -- & $\cdots$ & $-\cdot$ & -- & .01 \\
\hline w & 2.65 & $3-04-69$ & 0 & 6.9 & 47 & 70 & -- & -- & $-\cdot$ & -- & .01 \\
\hline$w$ & 265 & $6-24-69$ & 0 & 6.7 & 32 & 54 & -- & -- & $\cdots$ & -- & .01 \\
\hline w & 265 & $9-10-69$ & 5 & 6.8 & 45 & 60 & -- & - & -- & -- & .04 \\
\hline$w$ & 265 & $3-03-70$ & 8 & 6.9 & 35 & 56 & -- & - & -- & $\because$ & .03 \\
\hline$w$ & 265 & $6-16-70$ & 2 & 6.5 & 26 & 48 & -- & -- & 11 & - & .01 \\
\hline w & 265 & $9-22-70$ & 5 & 7.0 & 56 & 68 & - & $\cdots$ & 15 & $\cdots$ & .01 \\
\hline$w$ & 265 & $3-15-71$ & 3 & 6.9 & 54 & 70 & -- & $\cdots$ & 19 & -- & .00 \\
\hline w & 265 & $7-12-71$ & 3 & 6.7 & 56 & 70 & -- & -- & 20 & -- & .01 \\
\hline w & 265 & $3-21-72$ & 0 & 7.0 & 50 & 63 & 20 & 3.5 & 18 & 1 & .02 \\
\hline w & 265 & $7-17-72$ & 0 & 6.6 & 57 & 58 & 18 & 3.4 & 21 & 1.2 & .02 \\
\hline$w$ & 265 & $10-16-72$ & 5 & 6.7 & 44 & 66 & 21 & 3.2 & 15 & 1.1 & .01 \\
\hline$w$ & 265 & $7-23-73$ & 0 & 6.9 & 52 & 81 & 27 & 3.3 & 10 & 1 & .02 \\
\hline w & 265 & $3-25-74$ & 0 & 6.9 & 75 & 69 & 20 & 4.6 & 25 & 1.5 & .00 \\
\hline$w$ & 265 & $7-23-74$ & 0 & 6.8 & 67 & 78 & 25 & 3.8 & 17 & 1.2 & .00 \\
\hline$w$ & 266 & $6-08-71$ & 5 & 6.7 & 62 & 60 & -- & -- & - & -- & .01 \\
\hline$w$ & 266 & $3-19-73$ & 0 & 6.7 & 31 & 47 & 14 & 2.8 & 10 & .8 & .00 \\
\hline$w$ & 266 & $7-23-74$ & 0 & 6.8 & 70 & 77 & 25 & 3.7 & 16 & $1 \cdot 2$ & .00 \\
\hline$w$ & 266 & $11-18-74$ & 0 & 7.0 & 61 & 73 & 23 & 3.8 & 16 & 1.4 & .00 \\
\hline$w$ & 313 & $11-28-72$ & 5 & 6.6 & 36 & 48 & $=$ & -- & $\therefore$ & -- & .03 \\
\hline$w$ & 313 & $1-14-74$ & 5 & 6.8 & 43 & 49 & 15 & 2.6 & 10 & 0.6 & .07 \\
\hline$w$ & 313 & $3-25-74$ & 0 & 6.8 & 71 & 69 & 20 & 4.6 & 24 & 1.5 & .00 \\
\hline w & 313 & $7-23-74$ & 0 & 6.7 & 52 & 60 & 19 & 3.2 & 10 & 0.8 & .00 \\
\hline$w$ & 313 & $11-18-74$ & 0 & 6.8 & 55 & 59 & 18 & $3 \cdot 4$ & 13 & 0.8 & .00 \\
\hline & & & & & & & & & & SWAN & \\
\hline W & 5 & $8-02-60$ & 2 & 6.0 & 9 & 62 & -- & - & $\cdots$ & -. & .00 \\
\hline$w$ & 5 & $12-01-60$ & 3 & 5.7 & 5 & 34 & -- & -- & -- & -- & .00 \\
\hline$w$ & 5 & $7-31-61$ & 5 & 5.5 & 7 & 58 & -- & -- & -- & -- & .01 \\
\hline
\end{tabular}


Table 3.--Chemical analyses of ground water (continued)

\begin{tabular}{|c|c|c|c|c|c|c|c|c|c|c|c|c|c|c|c|c|c|}
\hline $\begin{array}{c}\mathrm{L} \propto \mathrm{c} 1 \\
\text { well } \\
\text { number }\end{array}$ & $\begin{array}{c}\text { Date } \\
\text { of } \\
\text { sample }\end{array}$ & Col or $\mathrm{pH}$ & $\begin{array}{c}\text { Alka- } \\
1 \text { inity } \\
\text { as } \\
\mathrm{CaCO}_{3} \\
(\mathrm{mg} / \mathrm{L})\end{array}$ & $\begin{array}{l}\text { Hard- } \\
\text { ness } \\
(\mathrm{mg} / \mathrm{L})\end{array}$ & $\begin{array}{l}\mathrm{Cal-} \\
\text { cium } \\
(\mathrm{mg} / \mathrm{L})\end{array}$ & $\begin{array}{l}\text { Magne- } \\
\text { sium } \\
(\mathrm{mg} / \mathrm{L})\end{array}$ & $\begin{array}{l}\text { Sod i um } \\
(\mathrm{mg} / \mathrm{L})\end{array}$ & $\begin{array}{l}\text { Potas- } \\
\text { sium } \\
(\mathrm{mg} / \mathrm{L})\end{array}$ & $\begin{array}{c}\text { Iron } \\
(\mathrm{mg} / \mathrm{L})\end{array}$ & $\begin{array}{c}\text { Man- } \\
\text { ganese } \\
(\mathrm{mg} / \mathrm{L})\end{array}$ & $\begin{array}{l}\text { Silica } \\
(\mathrm{mg} / \mathrm{L})\end{array}$ & $\begin{array}{l}\text { Sul- } \\
\text { fate } \\
(\mathrm{mg} / \mathrm{L})\end{array}$ & $\begin{array}{l}\text { Chlo- } \\
\text { ride } \\
(\mathrm{mg} / \mathrm{L})\end{array}$ & $\begin{array}{c}\text { Specific } \\
\text { conduct - } \\
\text { ance } \\
\text { (micro- } \\
\text { mhos) }\end{array}$ & $\begin{array}{l}\mathrm{Ni}- \\
\text { trate } \\
(\mathrm{mg} / \mathrm{L})\end{array}$ & $\begin{array}{l}\text { Copper } \\
(\mathrm{mg} / \mathrm{L})\end{array}$ & $\begin{array}{c}\text { Source } \\
\text { of } \\
\text { data }\end{array}$ \\
\hline
\end{tabular}

SWANSEA (c ont inued)

\begin{tabular}{|c|c|c|c|c|c|}
\hline 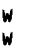 & $\begin{array}{l}5 \\
5\end{array}$ & $\begin{array}{r}12-04-61 \\
8-01-62\end{array}$ & $\begin{array}{l}5 \\
5\end{array}$ & $\begin{array}{l}6.2 \\
6.1\end{array}$ & 8 \\
\hline & 5 & $12-05-62$ & 5 & 6.2 & 6 \\
\hline & 5 & $4-05-63$ & 5 & $\cdot 8$ & 0 \\
\hline & 5 & $8-13-63$ & 5 & .2 & 13 \\
\hline & 5 & $12-04-63$ & 5 & 5.9 & \\
\hline & 5 & $4-03-64$ & 5 & 6.0 & \\
\hline & $\begin{array}{l}5 \\
5\end{array}$ & $\begin{array}{r}7-28-64 \\
11-09-64\end{array}$ & $\begin{array}{r}10 \\
5\end{array}$ & $\begin{array}{l}6.5 \\
6.5\end{array}$ & \\
\hline 6 & 5 & $2-23-65$ & 3 & 6.0 & \\
\hline H & 5 & $\begin{array}{l}5-26-65 \\
8-10-65\end{array}$ & 5 & & \\
\hline $\mathbf{W}$ & $\begin{array}{l}5 \\
5\end{array}$ & $\begin{array}{l}8-19-65 \\
2-17-66\end{array}$ & & 6.1 & 11 \\
\hline$w$ & 5 & $6-13-66$ & & 5.9 & \\
\hline $\mathbf{w}$ & 5 & $9-14$ & 0 & & \\
\hline$W$ & & $2-2$ & & & 0 \\
\hline$w$ & 5 & $9-1$ & & 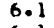 & \\
\hline$w$ & 5 & $6-14$ & & 6.1 & 0 \\
\hline$w$ & 5 & $6-06$ & & 6.0 & 4 \\
\hline$w$ & 5 & $8-21$ & & 6.0 & 11 \\
\hline $\mathbf{W}$ & & $3-18$ & & 5.8 & \\
\hline W & 5 & $7-05$ & & .1 & \\
\hline$w$ & 5 & $9-1$ & & 6.5 & 2 \\
\hline 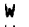 & 5 & $3-1$ & & 6.2 & \\
\hline$w$ & 5 & $9-29-70$ & & 6.0 & \\
\hline & 5 & $3-17$ & & .5 & \\
\hline$w$ & $\begin{array}{l}5 \\
5\end{array}$ & $\begin{array}{l}3-1 \\
7-1\end{array}$ & & 1 & \\
\hline$w$ & 5 & $10-26-72$ & & .2 & 3 \\
\hline$w$ & 5 & $3-26-73$ & & 6.3 & \\
\hline$w$ & 5 & $8-06-73$ & & 5.3 & \\
\hline$w$ & 5 & $11-15-73$ & & 6.3 & \\
\hline$w$ & 5 & $4-01-74$ & & .3 & \\
\hline$w$ & 5 & $11-26-74$ & 0 & 6.2 & \\
\hline$w$ & $4 R$ & $1-19-72$ & 0 & 6.4 & \\
\hline$w$ & 51 & $1-19-72$ & & 6.5 & \\
\hline$w$ & 52 & $1-13$ & & 6.5 & \\
\hline$w$ & 59 & $9-23$ & & 6.3 & \\
\hline w & 61 & $7-20-71$ & 5 & 6.5 & \\
\hline$w$ & 64 & $7-10-71$ & 0 & •4 & \\
\hline$w$ & 78 & $11-23-57$ & 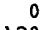 & 5.9 & \\
\hline$w$ & 79 & $12-16-57$ & 20 & .9 & \\
\hline$w$ & 84 & $11-30-57$ & & 1.9 & \\
\hline$w$ & 85 & $12-20-57$ & 0 & .1 & \\
\hline$w$ & 97 & $1-04-58$ & c & .0 & \\
\hline$w$ & 196 & $9-13-69$ & 5 & .3 & \\
\hline$w$ & 200 & $\begin{array}{l}9-22-69 \\
9-23-69\end{array}$ & 75 & .5 & \\
\hline$w$ & $\begin{array}{l}201 \\
206\end{array}$ & $\begin{array}{l}9-23-69 \\
9-26-69\end{array}$ & $\begin{array}{r}80 \\
5\end{array}$ & 7.4 & \\
\hline$w$ & 213 & $10-03-69$ & 0 & 6.6 & \\
\hline$w$ & 213 & $\begin{array}{l}4-0 \\
7-3\end{array}$ & 15 & & \\
\hline$w$ & 213 & $11-26-74$ & (S & $\begin{array}{l}6.2 \\
6.1\end{array}$ & \\
\hline 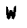 & 223 & $10-14-69$ & 30 & .0 & \\
\hline$w$ & 225 & $10-16-69$ & 15 & 6.5 & \\
\hline$w$ & 230 & $10-21-69$ & & 6.2 & \\
\hline$\omega$ & 239 & $9-23-74$ & 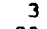 & 7 & \\
\hline$w$ & $\begin{array}{l}241 \\
241\end{array}$ & $\begin{array}{r}8-2 \\
12-\end{array}$ & $\begin{array}{l}30 \\
20\end{array}$ & $\begin{array}{l}3.8 \\
5.4\end{array}$ & \\
\hline$w$ & 241 & 6 & 7 & .3 & \\
\hline$w$ & 241 & .72 & 15 & 6.2 & \\
\hline$w$ & 241 & $10-26-72$ & 15 & 6.3 & \\
\hline$w$ & 241 & & & 6.3 & \\
\hline$w$ & 244 & $11-$ & 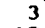 & 5.9 & \\
\hline 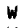 & 250 & & 15 & 6 & \\
\hline$w$ & 250 & $9-2$ & 10 & 6.1 & \\
\hline$w$ & 250 & $3-1$ & & 5 & \\
\hline$w$ & 250 & -72 & 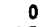 & 6.3 & \\
\hline$w$ & 250 & & 15 & .2 2 & \\
\hline$w$ & 250 & $10-2$ & 28 & 6 & \\
\hline$n$ & 250 & & & .4 & 7 \\
\hline$w$ & 250 & $8-06-73$ & 50 & 6.8 & \\
\hline$w$ & 250 & $11-15-73$ & 60 & 6.5 & 22 \\
\hline$w$ & 250 & & & 6 & 36 \\
\hline$w$ & 250 & $11-2$ & 2 & 6.3 & \\
\hline$w$ & 251 & & & 5.5 & \\
\hline$w$ & 251 & $12-01-60$ & 5 & 5. & \\
\hline$W$ & $\begin{array}{l}251 \\
251\end{array}$ & & & 5. & \\
\hline 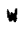 & $25 i$ & $12-$ & & 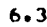 & \\
\hline w & 251 & $8-0$ & & & \\
\hline$w$ & 251 & $12-05-62$ & 10 & & \\
\hline w & 251 & $4-0$ & & & \\
\hline & 251 & $3-13-63$ & & 6 & \\
\hline 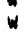 & 25 & $\begin{array}{r}12-04-63 \\
4-03-64\end{array}$ & 3 & 6 & \\
\hline & 2 & $7-28-64$ & & & \\
\hline & 23 & $11-05$ & & & \\
\hline & & & & & \\
\hline
\end{tabular}

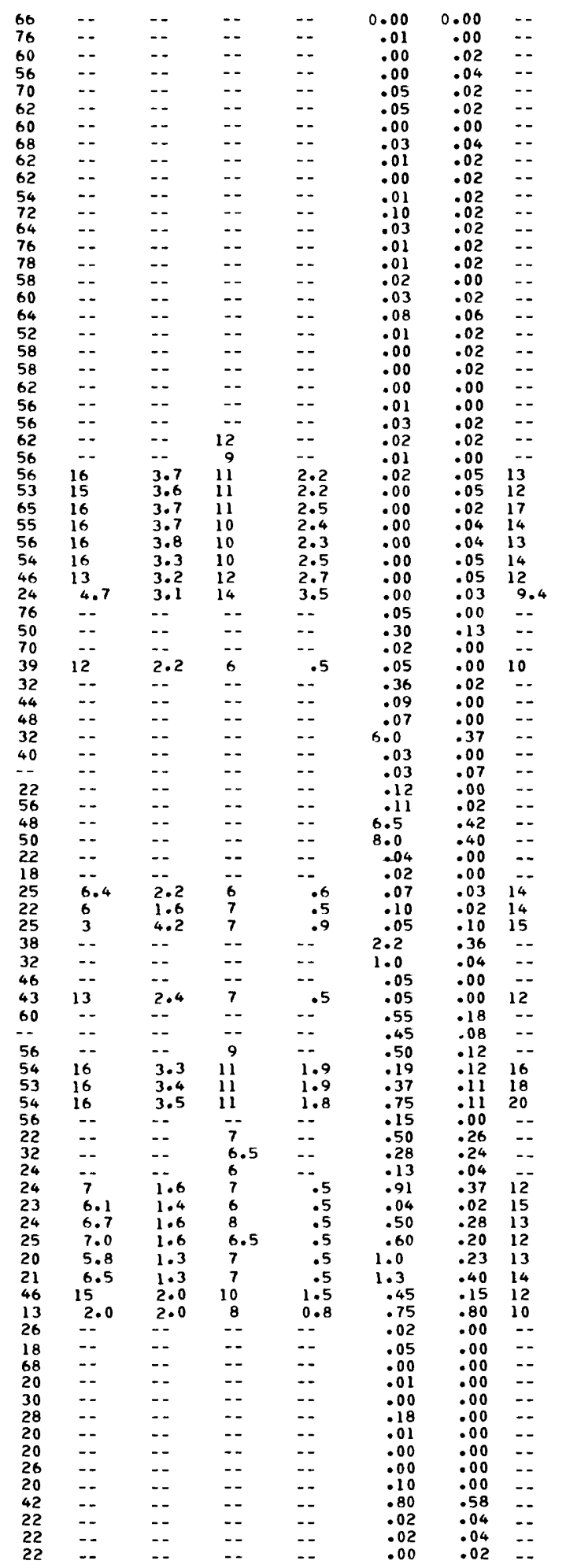

\begin{tabular}{|c|c|c|c|c|c|}
\hline-- & 7.5 & -- & 2 & - & 3 \\
\hline$\cdots$ & 15 & $\cdots$ & 2 & $=-$ & 3 \\
\hline-- & 12 & $=-$ & 2.8 & - & 3 \\
\hline$\cdots$ & 18 & -- & 3.6 & -. & 3 \\
\hline- & 15 & $\cdots$ & 3 & -- & 3 \\
\hline$\cdots$ & 18 & $\cdots$ & 3.2 & -- & 3 \\
\hline$\cdots$ & 14 & -- & 3.5 & -- & 3 \\
\hline$\cdots$ & 13 & -- & 4.3 & - & 3 \\
\hline-- & 15 & -- & 1.6 & - & 3 \\
\hline-- & 16 & -- & 3.5 & $\cdots$ & 3 \\
\hline-- & 16 & -- & 3.6 & $\cdots$ & 3 \\
\hline-- & 12 & $\cdots$ & .3 & -- & 3 \\
\hline$\cdots$ & 14 & $\cdots$ & .3 & -- & 3 \\
\hline-- & 14 & $\cdots$ & - & -- & 3 \\
\hline-- & 14 & -- & 3.8 & -- & 3 \\
\hline-- & 12 & -- & 1.3 & -- & 3 \\
\hline-- & 14 & -- & 4.5 & -- & 3 \\
\hline-- & 12 & $\cdots$ & 1.8 & - & 3 \\
\hline-- & 13 & -- & 4 & -. & 3 \\
\hline - & 15 & $\cdots$ & 5 & - & 3 \\
\hline$\cdots$ & 15 & $\cdots$ & 6 & -- & 3 \\
\hline -- & 17 & - & 5.3 & -- & 3 \\
\hline -- & 17 & -- & 3 & -- & 3 \\
\hline -- & 16 & $\cdots$ & 2.3 & $\cdots$ & 3 \\
\hline$\cdots$ & 17 & -- & 3 & $\cdots$ & 3 \\
\hline -- & 14 & -- & 2.1 & -- & 3 \\
\hline $\begin{array}{l}38 \\
38\end{array}$ & $\begin{array}{l}\text { is } \\
17\end{array}$ & $\begin{array}{l}180 \\
180\end{array}$ & $\begin{array}{l}2.5 \\
2.2\end{array}$ & $\overline{0.02}$ & $\begin{array}{l}3 \\
3\end{array}$ \\
\hline 43 & 15 & 180 & 2.6 & .03 & 3 \\
\hline 39 & 17 & 170 & 2.7 & .04 & 3 \\
\hline 36 & 18 & 185 & 2.8 & .04 & 3 \\
\hline 30 & 19 & 100 & 2.6 & .01 & 3 \\
\hline 0 & 19 & 175 & 2.5 & .03 & 3 \\
\hline 34 & 23 & 195 & 3.8 & .03 & 3 \\
\hline-- & 15 & -- & 0.1 & -- & 3 \\
\hline -- & 9.5 & -- & 0.1 & -- & 3 \\
\hline-- & 25 & -- & 1.6 & -- & 3 \\
\hline 20 & 8.0 & 110 & 1.0 & .00 & 3 \\
\hline -- & 9.5 & -- & 0.2 & -- & 3 \\
\hline-- & 11 & -- & 0.3 & -- & 3 \\
\hline-- & 15 & - & 2.6 & -- & 3 \\
\hline-- & 14 & -- & .12 & - & 3 \\
\hline-- & 7.2 & $\cdots$ & 3.5 & -- & 3 \\
\hline-- & 7.4 & -- & .07 & -- & 3 \\
\hline -- & 5.4 & -- & .70 & -- & 3 \\
\hline -.- & 14 & -- & 5.1 & -- & 3 \\
\hline -- & 12 & -- & 0.0 & -- & 3 \\
\hline-- & 9 & -- & 0.0 & -- & 3 \\
\hline-- & 8.6 & -- & 0.0 & -- & 3 \\
\hline$\overline{8}$ & 4.0 & -- & 0.0 & -- & 3 \\
\hline $\begin{array}{r}18 \\
4\end{array}$ & 6 & 90 & .3 & .03 & 3 \\
\hline 54 & $\begin{array}{l}5 \\
6\end{array}$ & $\begin{array}{r}82 \\
155\end{array}$ & .3 & $\begin{array}{l}.12 \\
.05\end{array}$ & 3 \\
\hline -- & 5.0 & - & 0.0 & $\ldots$ & $\begin{array}{l}3 \\
3\end{array}$ \\
\hline- & 1.5 & -- & 0.0 & -- & 3 \\
\hline -- & 21 & -- & .1 & -- & 3 \\
\hline 22 & 9.0 & 118 & .4 & .00 & 3 \\
\hline - & 12 & -- & .5 & -- & 3 \\
\hline-- & -- & $\cdots$ & -- & -- & 3 \\
\hline-- & 20 & -- & .9 & -- & \\
\hline 40 & 17 & 175 & .6 & .02 & 3 \\
\hline 40 & 18 & 170 & .6 & .00 & 3 \\
\hline 44 & 19 & 170 & .6 & .01 & 3 \\
\hline -- & $8 \cdot 8$ & -- & 3.5 & -- & 3 \\
\hline -- & 11 & -- & .1 & $\cdots$ & 3 \\
\hline -- & 8.5 & -- & -1 & $\cdots$ & 3 \\
\hline 12 & 7 & 84 & $\cdot 2$ & $=$ & 3 \\
\hline 11 & $\begin{array}{l}7.5 \\
1\end{array}$ & $\begin{array}{l}84 \\
78\end{array}$ & $\begin{array}{l}.2 \\
.2\end{array}$ & .85 & $\begin{array}{l}3 \\
3\end{array}$ \\
\hline 9 & 7 & 86 & .1 & .22 & 3 \\
\hline 11 & 6.5 & 82 & .2 & .05 & 3 \\
\hline 13 & 6.5 & 92 & .2 & .22 & 3 \\
\hline 9 & 8 & 76 & .1 & .22 & 3 \\
\hline 20 & 13 & 150 & 1.3 & .12 & 3 \\
\hline 26 & 7 & 112 & .1 & .20 & 3 \\
\hline -- & 3.5 & -- & .1 & -- & 3 \\
\hline-- & 4 & -- & $\cdot 1$ & -- & 3 \\
\hline-- & 12 & -- & -- & -- & 3 \\
\hline-- & 7 & -- & .5 & $\cdots$ & 3 \\
\hline- & 5.5 & -- & 0.0 & -- & 3 \\
\hline-- & 5.5 & $=$ & $\begin{array}{l}0.0 \\
0.0\end{array}$ & -- & 3 \\
\hline-- & $\begin{array}{l}3 \\
7.5\end{array}$ & -- & $\begin{array}{l}0.0 \\
0.0\end{array}$ & $\ldots$ & 3 \\
\hline- & $7^{103}$ & - & 0.0 & - & 3 \\
\hline$=$ & 7.5 & -- & .1 & -- & 3 \\
\hline & 9.5 & -- & .2 & -- & 3 \\
\hline-- & 5 & -- & .3 & $\cdots$ & 3 \\
\hline 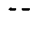 & $\begin{array}{l}5 \\
5.5\end{array}$ & -- & 1 & -- & 3 \\
\hline-- & 5.5 & -- & - I & $\cdots$ & 3 \\
\hline
\end{tabular}


Table 3.--Chemical analyses of ground water (continued)

\begin{tabular}{|c|c|c|c|c|c|c|c|c|c|c|c|c|c|c|c|c|c|c|}
\hline $\begin{array}{c}\text { Local } \\
\text { well } \\
\text { number }\end{array}$ & $\begin{array}{c}\text { Date } \\
\text { of } \\
\text { sample }\end{array}$ & Col or & $\mathrm{pH}$ & $\begin{array}{c}\text { Alka- } \\
\text { linity } \\
\text { as } \\
\mathrm{CaCO}_{3} \\
(\mathrm{mg} / \mathrm{L})\end{array}$ & $\begin{array}{l}\text { Hard- } \\
\text { ness } \\
(\mathrm{mg} / \mathrm{L})\end{array}$ & $\begin{array}{l}\text { Cal- } \\
\text { cium } \\
\text { (mg/L) }\end{array}$ & $\begin{array}{l}\text { Magne- } \\
\text { sium } \\
\text { (mg/L) }\end{array}$ & $\begin{array}{l}\text { Sodium } \\
(\mathrm{mg} / \mathrm{L})\end{array}$ & $\begin{array}{l}\text { Potas- } \\
\text { si um } \\
\text { (mg/L) }\end{array}$ & $\begin{array}{c}\text { Ir on } \\
(\mathrm{mg} / \mathrm{L})\end{array}$ & $\begin{array}{c}\text { Man- } \\
\text { ganese } \\
(m g / L)\end{array}$ & $\begin{array}{l}\text { Silica } \\
\text { (mg/L) }\end{array}$ & $\begin{array}{l}\text { Sul- } \\
\text { fate } \\
(\mathrm{mg} / \mathrm{L})\end{array}$ & $\begin{array}{l}\text { Chlo- } \\
\text { ride } \\
\text { (mg/L) }\end{array}$ & $\begin{array}{c}\text { Specific } \\
\text { conduct - } \\
\text { ance } \\
\text { (micro- } \\
\text { mhos) }\end{array}$ & $\begin{array}{c}\mathrm{Ni}- \\
\text { trate } \\
(\mathrm{mg} / \mathrm{L})\end{array}$ & $\begin{array}{l}\text { Copper } \\
\text { (mg/L) }\end{array}$ & $\begin{array}{c}\text { Source } \\
\text { of } \\
\text { data }\end{array}$ \\
\hline
\end{tabular}

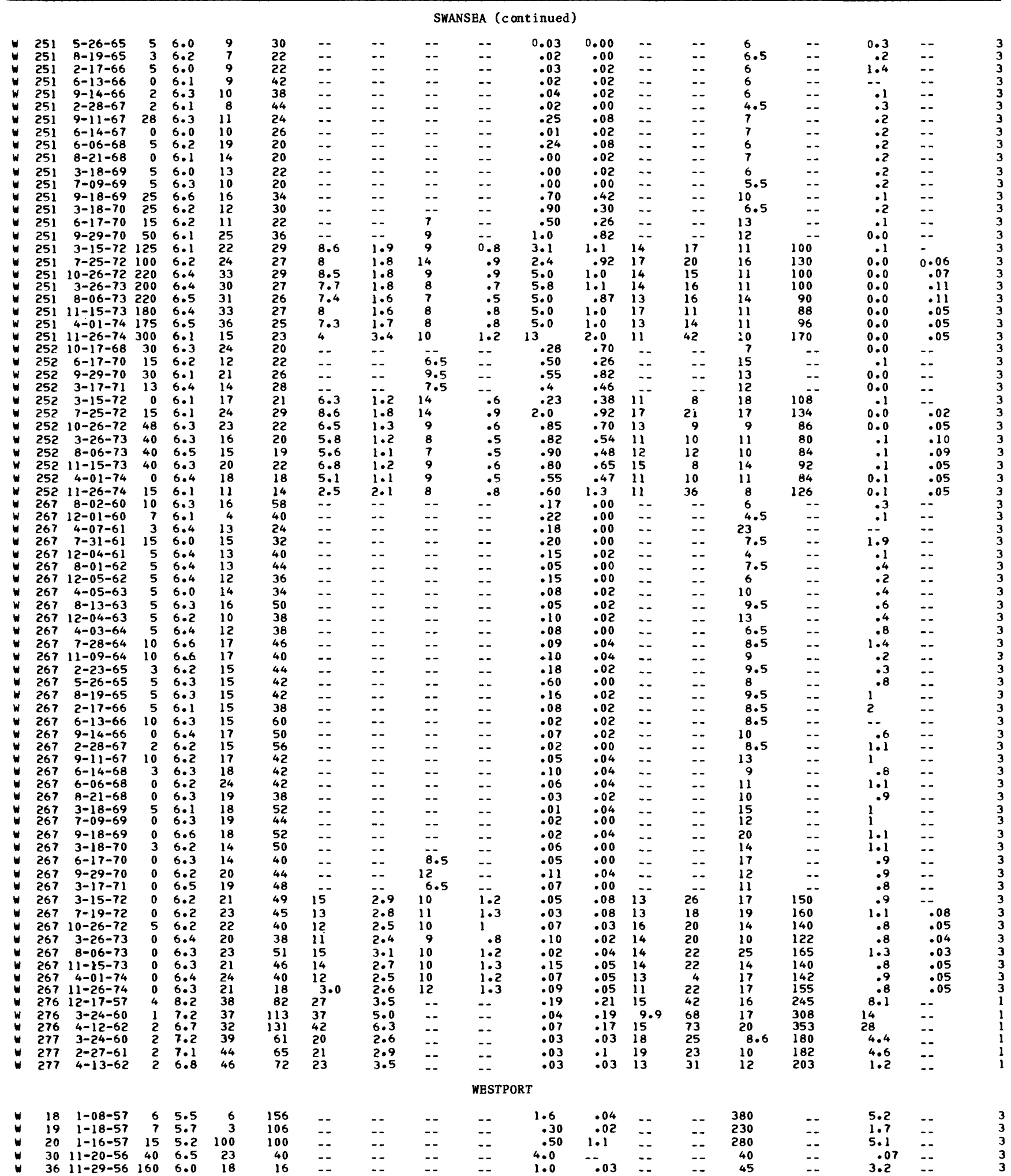


Table 3.--Chemical analyses of ground water (continued)

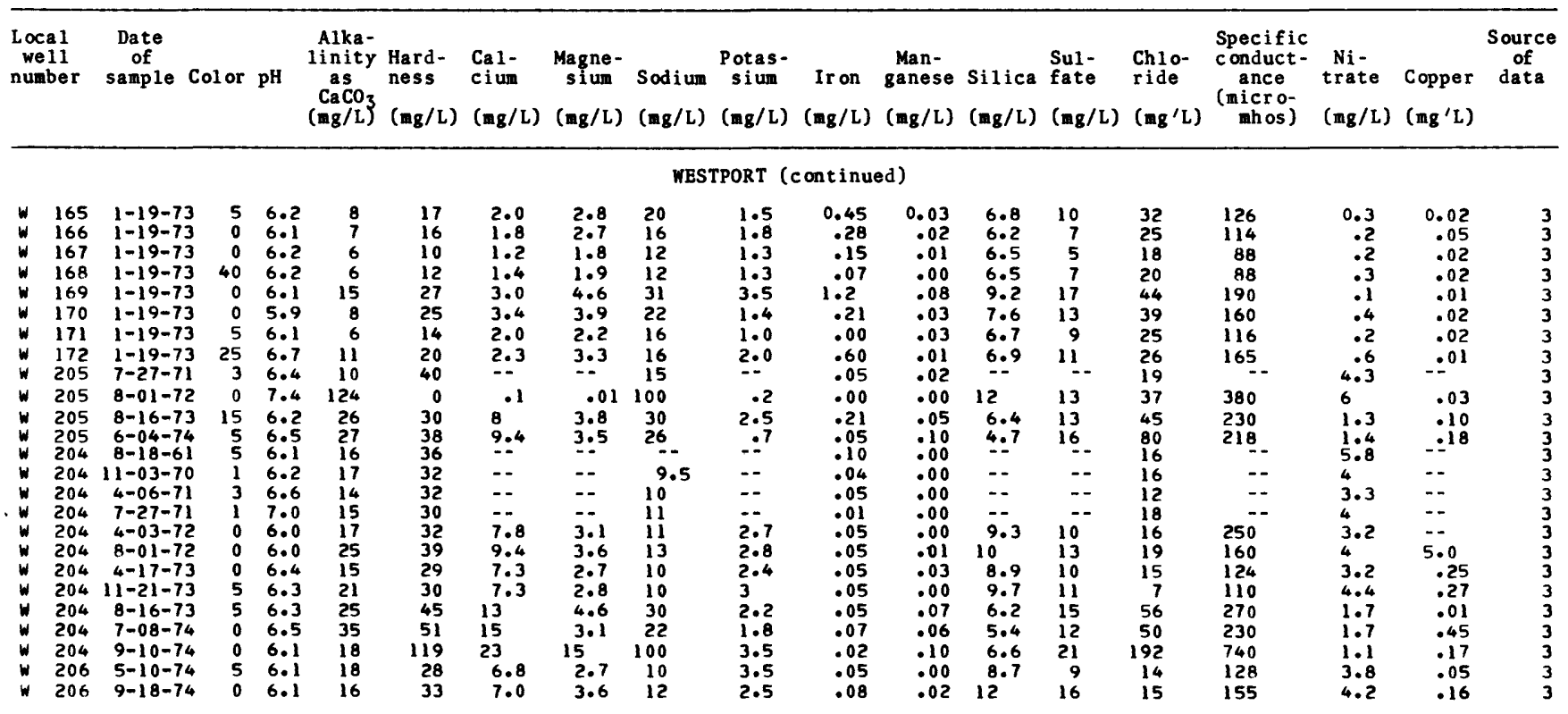


Table 4.-Records available in other reports at gaging stations

\begin{tabular}{|c|c|c|}
\hline Station number & 01106000 & 01109200 \\
\hline Station name & $\begin{array}{l}\text { Adamsville Brook } \\
\text { at Adamsville, R.I. }\end{array}$ & $\begin{array}{l}\text { West Branch Palmer River } \\
\text { near Rehoboth, Mass. }\end{array}$ \\
\hline Latitude & $41^{\circ} 33^{\prime} 30^{\prime \prime}$ & $41^{\circ} 52^{\prime} 46^{\prime \prime}$ \\
\hline Longitude & 71007471 & $71^{\circ} 15^{\prime} 18^{\prime \prime}$ \\
\hline Location (pl. I) & $\begin{array}{l}\text { Newport County, on right } \\
\text { bank } 0.2 \text { mile upstream } \\
\text { from milldam at Adamsville }\end{array}$ & $\begin{array}{l}\text { Bristol County, at } \\
\text { Homestead Ave., } \\
2.6 \text { miles north } \\
\text { of Rehoboth }\end{array}$ \\
\hline Drainage area $\left(\mathrm{mi}^{2}\right)$ & 7.91 & 4.96 \\
\hline Records available & $\begin{array}{l}\text { October 1940- } \\
\text { September } 1978\end{array}$ & $\begin{array}{l}\text { October 1962- } \\
\text { September } 1974\end{array}$ \\
\hline Remarks & $\begin{array}{l}\text { Specific conductance and } \\
\text { water temperature records } \\
\text { for February } 1973 \text { to } \\
\text { April } 1974\end{array}$ & $\begin{array}{l}\text { Specific conductance and } \\
\text { water temperature records } \\
\text { for February } 1973 \text { to } \\
\text { April } 1974\end{array}$ \\
\hline Information sources & $\begin{array}{l}\text { U.S. Geological Survey, } \\
\text { 1954, 1964, 1969a, 1969b, } \\
\text { 1970, 1971, 1973, 1975a, } \\
\text { 1975b, 1975c, 1976, 1977, } \\
\text { 1978, 1979 }\end{array}$ & $\begin{array}{l}\text { U.S. Geological Survey, } \\
\text { 1964, 1969a, 1969b, 1970, } \\
\text { 1971, 1973, 1974, 1975a, } \\
\text { 1975b, 1975c }\end{array}$ \\
\hline
\end{tabular}


Table 5.--Discharge, specific conductance, and water temperature measurements at partial-record stations during water years 1972-74

(Complete chemical analyses in table 6 for dates marked by asterisk.)

\begin{tabular}{|c|c|c|c|c|c|c|}
\hline $\begin{array}{l}\text { Station } \\
\text { name } \\
\text { and } \\
\text { number }\end{array}$ & $\begin{array}{l}\text { Location } \\
\text { (See plate 1.) }\end{array}$ & $\begin{array}{l}\text { Drain- } \\
\text { age } \\
\text { area } \\
\left(m i^{2}\right)\end{array}$ & - & $\begin{array}{c}\text { Dis- } \\
\text { charge } \\
\left(\mathrm{ft}^{3} / \mathrm{s}\right)\end{array}$ & $\begin{array}{l}\text { Specific } \\
\text { conduct- } \\
\text { ance } \\
\text { (micro- } \\
\text { mos at } \\
25^{\circ} \mathrm{C} \text { ) }\end{array}$ & $\begin{array}{l}\text { Water } \\
\text { tem- } \\
\text { pera- } \\
\text { ture } \\
\left({ }^{\circ} \mathrm{C}\right)\end{array}$ \\
\hline $\begin{array}{l}\text { Shingle Is land } \\
\text { River near } \\
\text { North } \\
\text { Dartmouth } \\
01105937\end{array}$ & $\begin{array}{l}\text { Lat } 41040 \text { '50", long } 71001 \text { '05", } \\
\text { Bristol County, at bridge on } \\
\text { 01d Fall River Road, } 3 \text { miles } \\
\text { northwest of North Dartmouth }\end{array}$ & 8.31 & $\begin{array}{r}8-24-72 \\
6-7-73 \\
\star 8-9-73 \\
9-6-73 \\
7-18-74 \\
8-19-74\end{array}$ & $\begin{array}{r}1.44 \\
6.52 \\
1.95 \\
1.69 \\
2.13 \\
.04\end{array}$ & $\begin{array}{l}67 \\
50 \\
66 \\
60 \\
55 \\
--\end{array}$ & $\begin{array}{l}19 \\
16 \\
21 \\
21 \\
18 \\
--\end{array}$ \\
\hline $\begin{array}{l}\text { Shingle Island } \\
\text { River at } \\
\text { Hixville Road } \\
\text { near North } \\
\text { Dartmouth } \\
01105943\end{array}$ & $\begin{array}{l}\text { Lat } 41040 \text { '10", long } 71^{\circ} 01 \text { ' } 32 " \text { ", } \\
\text { Bristol County, at culvert on } \\
\text { Hixville Road, } 3 \text { miles } \\
\text { northwest of North Dartmouth }\end{array}$ & 18.1 & $\begin{array}{r}8-24-72 \\
* 10-12-72 \\
6-7-73 \\
* 8-9-73 \\
9-6-73 \\
7-18-74 \\
8-19-74\end{array}$ & $\begin{array}{l}4.41 \\
23.5 \\
23.6 \\
5.07 \\
4.89 \\
6.54 \\
3.33\end{array}$ & $\begin{array}{l}74 \\
60 \\
57 \\
77 \\
79 \\
71 \\
70\end{array}$ & $\begin{array}{l}19 \\
10 \\
18 \\
22 \\
21 \\
19 \\
22\end{array}$ \\
\hline $\begin{array}{l}\text { Bread and } \\
\text { Cheese Brook } \\
\text { at Head of } \\
\text { Westport } \\
01105947\end{array}$ & $\begin{array}{l}\text { Lat } 41^{\circ} 38^{\prime} 00^{\prime \prime}, \text { long } 71^{\circ} 03^{\prime} 46^{\prime \prime} \text {, } \\
\text { Bristol County, at culvert on } \\
\text { State Highway } 177,1 \text { mile } \\
\text { north of Head of Westport }\end{array}$ & 8.70 & $\begin{array}{r}8-24-72 \\
\star 10-12-72 \\
6-7-73 \\
8-9-73 \\
9-6-73 \\
7-18-74 \\
8-19-74\end{array}$ & $\begin{array}{r}.91 \\
11.8 \\
6.82 \\
1.78 \\
1.47 \\
1.72 \\
.18\end{array}$ & $\begin{array}{l}142 \\
105 \\
106 \\
134 \\
125 \\
128 \\
136\end{array}$ & $\begin{array}{r}18 \\
9 \\
17 \\
24 \\
23 \\
18 \\
22\end{array}$ \\
\hline $\begin{array}{l}\text { Kirby Brook } \\
\text { near Head } \\
\text { of Westport } \\
01105950\end{array}$ & $\begin{array}{l}\text { Lat } 41^{\circ} 36^{\prime} 02 " \text {, long } 71^{\circ} 04^{\prime} 25 " \text {, } \\
\text { Bristol County, at culvert on } \\
\text { Drift Road, } 1.5 \text { miles south of } \\
\text { Head of Westport }\end{array}$ & 3.69 & $\begin{array}{r}8-24-72 \\
6-7-73 \\
\star 8-9-73 \\
9-6-73 \\
7-17-74 \\
8-19-74\end{array}$ & $\begin{array}{l}.04 \\
1.75 \\
.20 \\
.04 \\
.05\end{array}$ & $\begin{array}{r}152 \\
106 \\
118 \\
138 \\
132 \\
--\end{array}$ & $\begin{array}{l}18 \\
17 \\
22 \\
22 \\
20 \\
--\end{array}$ \\
\hline $\begin{array}{l}\text { Angeline } \\
\text { Brook near } \\
\text { Westport } \\
\text { Point } \\
01106005\end{array}$ & $\begin{array}{l}\text { Lat } 41^{\circ} 33^{\prime} 05^{\prime \prime}, \text { long } 71^{\circ} 06^{\prime} 20^{\prime \prime} \text {, } \\
\text { Bristol County, at culvert } \\
\text { on Cornell Road, } 2.5 \text { miles } \\
\text { northwest of Westport Point }\end{array}$ & $3.22+$ & $\begin{array}{r}8-24-72 \\
\star 10-12-72 \\
6-7-73 \\
\star 8-9-73 \\
9-6-73 \\
7-17-74 \\
8-19-74\end{array}$ & $\begin{array}{l}.13 \\
2.95 \\
2.18 \\
.40 \\
.19 \\
.32\end{array}$ & $\begin{array}{r}140 \\
90 \\
112 \\
128 \\
130 \\
136 \\
--\end{array}$ & $\begin{array}{l}18 \\
11 \\
15 \\
21 \\
21 \\
18 \\
--\end{array}$ \\
\hline
\end{tabular}


Table 5.--Discharge, specific conductance, and water temperature measurements at partial-record stations during water years 1972-74 (continued)

\begin{tabular}{|c|c|c|c|c|c|c|}
\hline $\begin{array}{l}\text { Station } \\
\text { name } \\
\text { and } \\
\text { number }\end{array}$ & $\begin{array}{l}\text { Location } \\
\text { (See plate 1.) }\end{array}$ & $\begin{array}{l}\text { Drain } \\
\text { age } \\
\text { area } \\
\left(m i^{2}\right)\end{array}$ & 1- & $\begin{array}{c}\text { Dis- } \\
\text { charge } \\
\left(\mathrm{ft}^{3} / \mathrm{s}\right)\end{array}$ & $\begin{array}{l}\text { Specific } \\
\text { conduct- } \\
\text { ance } \\
\text { (micro- } \\
\text { mhos at } \\
25^{\circ} \mathrm{C} \text { ) }\end{array}$ & $\begin{array}{l}\text { Water } \\
\text { tem- } \\
\text { pera- } \\
\text { ture } \\
\left({ }^{\circ} \mathrm{C}\right)\end{array}$ \\
\hline $\begin{array}{l}\text { Lewin Brook } \\
\text { at Swansea } \\
01109130\end{array}$ & $\begin{array}{l}\text { Lat } 41044^{\prime} 55^{\prime \prime}, \text { long } 71011^{\prime} 45^{\prime \prime} \text {, } \\
\text { Bristol County, at culvert } \\
\text { on Stevens Road at Swansea }\end{array}$ & 2.83 & $\begin{array}{r}8-24-72 \\
6-7-73 \\
\star 8-10-73 \\
9-11-73 \\
7-18-74 \\
8-19-74\end{array}$ & $\begin{array}{r}0.07 \\
1.15 \\
.21 \\
.08 \\
.14 \\
.05\end{array}$ & $\begin{array}{r}120 \\
85 \\
99 \\
110 \\
102 \\
--\end{array}$ & $\begin{array}{l}19 \\
19 \\
22 \\
19 \\
19 \\
--\end{array}$ \\
\hline $\begin{array}{l}\text { Cole River } \\
\text { near } \\
\text { Swansea } \\
01109135\end{array}$ & $\begin{array}{l}\text { Lat } 41^{\circ} 46^{\prime} 30^{\prime \prime}, \text { long } 71^{\circ} 12^{\prime} 00^{\prime \prime}, \\
\text { Bristol County, at bridge on } \\
\text { Hortonville Road, } 2 \text { miles } \\
\text { north of Swansea }\end{array}$ & 7.79 & $\begin{array}{r}8-24-73 \\
* 10-12-72 \\
6-7-73 \\
* 8-10-73 \\
9-11-73 \\
7-18-74 \\
8-20-74\end{array}$ & $\begin{array}{r}.55 \\
9.59 \\
5.09 \\
1.36 \\
4.97 \\
.89 \\
.01\end{array}$ & $\begin{array}{l}71 \\
62 \\
59 \\
65 \\
91 \\
58 \\
--\end{array}$ & $\begin{array}{l}22 \\
11 \\
18 \\
22 \\
16 \\
20 \\
--\end{array}$ \\
\hline $\begin{array}{l}\text { East Branch } \\
\text { Palmer River } \\
\text { at Rehoboth } \\
01109195\end{array}$ & $\begin{array}{l}\text { Lat } 41050 \text { ' } 30^{\prime \prime}, \text { long } 71014 \text { ' } 35 " \text { ", } \\
\text { Bristo } 1 \text { County, at bridge on } \\
\text { State Highway 118, at } \\
\text { Rehoboth }\end{array}$ & 11.7 & $\begin{array}{r}8-25-72 \\
6-7-73 \\
\star 8-10-73 \\
9-11-73 \\
7-18-74 \\
8-20-74\end{array}$ & $\begin{array}{c}1.59 \\
22.0 \\
1.81 \\
12.8 \\
1.34 \\
2.15\end{array}$ & $\begin{array}{l}88 \\
63 \\
81 \\
65 \\
84 \\
60\end{array}$ & $\begin{array}{l}20 \\
18 \\
22 \\
16 \\
18 \\
20\end{array}$ \\
\hline $\begin{array}{l}\text { Palmer River } \\
\text { near } \\
\text { Rehoboth } \\
01109215\end{array}$ & $\begin{array}{l}\text { Lat } 41^{\prime 0} 49^{\prime} 50^{\prime \prime}, \text { long } 71^{\circ} 16^{\prime} 55^{\prime \prime} \text {, } \\
\text { Bristol County, at bridge on } \\
\text { Summer Street, } 2 \text { miles } \\
\text { southwest of Rehoboth }\end{array}$ & 23.0 & $\begin{array}{r}8-25-72 \\
* 10-12-72 \\
* 6-7-73 \\
* 8-10-73 \\
9-11-73 \\
7-18-74 \\
8-20-74\end{array}$ & $\begin{array}{c}5.77 \\
35.4 \\
77.6 \\
6.85 \\
28.1 \\
5.01 \\
4.08\end{array}$ & $\begin{array}{r}114 \\
76 \\
60 \\
102 \\
62 \\
106 \\
85\end{array}$ & $\begin{array}{l}19 \\
9.5 \\
18 \\
21 \\
16 \\
19 \\
19\end{array}$ \\
\hline $\begin{array}{l}\text { Rocky Run } \\
\text { near } \\
\text { Rehoboth } \\
01109225\end{array}$ & $\begin{array}{l}\text { Lat } 41046^{\prime} 52^{\prime \prime}, \text { long } 71^{\circ} 15^{\prime} 05^{\prime \prime} \\
\text { Bristol County, at culvert } \\
\text { on Davis Street, } 4 \text { miles } \\
\text { south of Rehoboth }\end{array}$ & 7.37 & $\begin{array}{r}8-24-72 \\
* 10-12-72 \\
6-7-73 \\
* 8-10-73 \\
9-11-73 \\
7-18-74 \\
8-20-74\end{array}$ & $\begin{array}{r}1.07 \\
6.73 \\
5.54 \\
1.14 \\
3.60 \\
1.41 \\
.02\end{array}$ & $\begin{array}{r}98 \\
65 \\
66 \\
83 \\
63 \\
77 \\
104\end{array}$ & $\begin{array}{l}24 \\
11 \\
21 \\
24 \\
17 \\
22 \\
20\end{array}$ \\
\hline $\begin{array}{l}\text { Runn ins River } \\
\text { at Seekonk } \\
01109270\end{array}$ & $\begin{array}{l}\text { Lat } 41049^{\prime} 25^{\prime \prime}, \text { long } 71020^{\prime} 00^{\prime \prime} \text {, } \\
\text { Bristol County, at culvert on } \\
\text { Pleasant Street, } 1 \text { mile north } \\
\text { of Seekonk }\end{array}$ & 4.24 & $\begin{array}{r}8-25-72 \\
6-7-73 \\
\star 8-10-73 \\
9-11-73 \\
7-17-74 \\
8-20-74\end{array}$ & $\begin{array}{r}.79 \\
3.68 \\
1.21 \\
4.56 \\
.42 \\
.18\end{array}$ & $\begin{array}{l}195 \\
140 \\
180 \\
110 \\
180 \\
225\end{array}$ & $\begin{array}{l}19 \\
19 \\
21 \\
16 \\
20 \\
17\end{array}$ \\
\hline
\end{tabular}


Table 6.--Chemical analyses at gaging stations and partial-record stations

\begin{tabular}{|c|c|c|c|c|c|c|c|c|c|c|c|c|c|c|c|}
\hline Date & Time & $\begin{array}{l}\text { Dis- } \\
\text { charge } \\
\left(\mathrm{ft}^{3} / \mathrm{s}\right)\end{array}$ & $\begin{array}{c}\text { Dis- } \\
\text { solved } \\
\text { silica } \\
(\mathrm{SiD}) \\
(\mathrm{mg} / \mathrm{L})\end{array}$ & $\begin{array}{c}\text { Total } \\
\text { iron } \\
\text { (Fe) } \\
\text { (ug/L) }\end{array}$ & $\begin{array}{c}\text { Total } \\
\text { man- } \\
\text { ganese } \\
(\mathrm{Mn}) \\
(\mathrm{ug} / \mathrm{L})\end{array}$ & $\begin{array}{l}\text { Dis } \\
\text { solved } \\
\text { cal- } \\
\text { cium } \\
\text { (Ca) } \\
\text { (mg/L) }\end{array}$ & $\begin{array}{c}\text { Dis- } \\
\text { solved } \\
\text { magne- } \\
\text { sium } \\
\text { (Mg) } \\
(\mathrm{mg} / \mathrm{L})\end{array}$ & $\begin{array}{c}\text { Dis- } \\
\text { solved } \\
\text { sodium } \\
(\mathrm{Na}) \\
(\mathrm{mg} / \mathrm{L})\end{array}$ & $\begin{array}{c}\text { Dis- } \\
\text { solved } \\
\text { potas- } \\
\text { sium } \\
(\mathrm{K}) \\
(\mathrm{mg} / \mathrm{L})\end{array}$ & $\begin{array}{l}\text { Bicar- } \\
\text { bonate } \\
\left(\mathrm{HCO}_{3}\right) \\
(\mathrm{mg} / \mathrm{L})\end{array}$ & $\begin{array}{l}\text { Car- } \\
\text { bon- } \\
\text { ate } \\
\left(\mathrm{Co}_{3}\right) \\
(\mathrm{mg} / \mathrm{L})\end{array}$ & $\begin{array}{l}\text { Alka- } \\
\text { linity } \\
\text { as } \\
\mathrm{CaCO}_{3} \\
(\mathrm{mg} / \mathrm{L})\end{array}$ & $\begin{array}{l}\text { Dis- } \\
\text { solved } \\
\text { sulfate } \\
\left(\mathrm{So}_{4}\right) \\
(\mathrm{mg} / \mathrm{L})\end{array}$ & $\begin{array}{l}\text { Dis- } \\
\text { solved } \\
\text { chlo- } \\
\text { ride } \\
\text { (Cl) } \\
\text { (mg/L) }\end{array}$ & $\begin{array}{l}\text { Dis- } \\
\text { solved } \\
\text { fluo- } \\
\text { ride } \\
(\mathrm{F}) \\
(\mathrm{mg} / \mathrm{L})\end{array}$ \\
\hline
\end{tabular}

East Branch Westport River basin

01105937 Shingle Island River at 01d Fall River Road (Lat 414050 Long 0710105.01 )

$\begin{array}{llllllllllllllll}08-09-73 & 1430 & 2.2 & 13 & 1800 & 90 & 2.0 & 1.4 & 5.3 & 1.5 & 6 & 0 & 5 & 7.2 & 8.0 & 0.4\end{array}$ 01105943 Shingle Island River at Hixville Road, North Dartmouth, Mass. (Lat 414010 Long 07101 32)

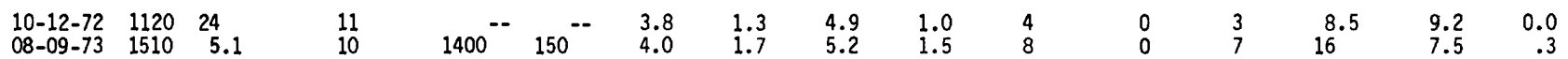
01105947 Bread and Cheese Brook at Head of Westport, Mass. (Lat 413800 Long 07103 46)

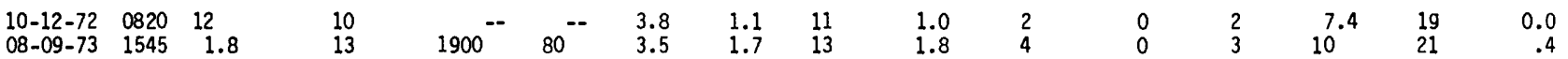
01105950 Kirby Brook near Head of Westport, Mass. (Lat 413602 Long 07104 25)

$.08-09-73 \quad 1615 \quad 0.20$

17

$\begin{array}{lll}5.0 & 2.0 \quad 11\end{array}$

$2.3 \quad 9$

$7 \quad 14$

$19 \quad 0.4$

West Branch Westport River basin

01106000 Adamsville Brook at Adamsville, R.I. (Lat 413330 Long 07107 47)

\begin{tabular}{llllllllllllllll}
$10-12-72$ & 0930 & 14 & 8.5 & \multirow{2}{*}{--} & -- & 4.1 & 1.1 & 7.6 & 1.6 & 6 & 0 & 5 & 6.0 & 13 & .0 \\
$08-09-73$ & 1720 & 2.5 & 16 & 1500 & 40 & 2.9 & 1.5 & 8.1 & 1.9 & 7 & 0 & 6 & 6.0 & 14 & .4
\end{tabular}

01106005 Angeline Brook near Westport Point, Mass. (Lat 413305 Long 0710620 )

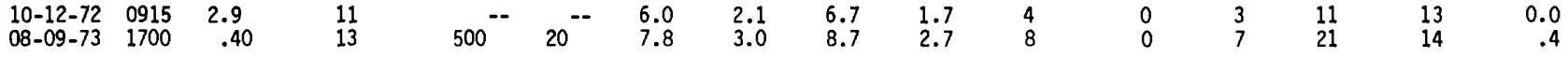

Lee River basin

01109130 Lewin Brook at Swansea, Mass (Lat 414455 Long 0711145.01 )

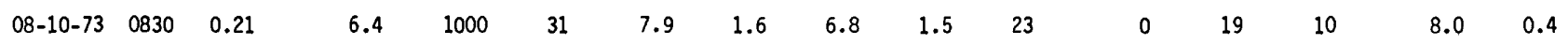

Cole River basin

01109135 Cole River near Swansea, Mass. (Lat 414630 Long 0711200 )

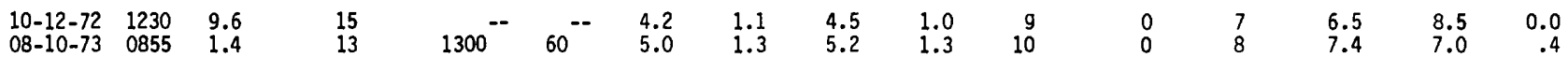

Palmer River basin

01109195 East Branch Palmer River at Rehoboth, Mass. (Lat 415030 Long 07114 35.01)

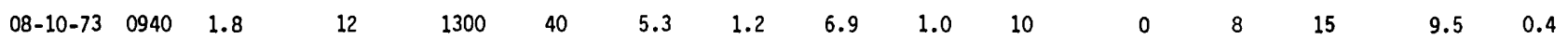
01109200 West Branch Palmer River near Rehoboth, Mass. (Lat 415246 Long 071 15 18)

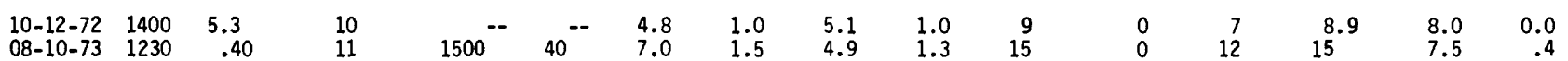
01109215 Palmer River near Rehoboth, Mass. (Lat 414950 Long 0711655 )

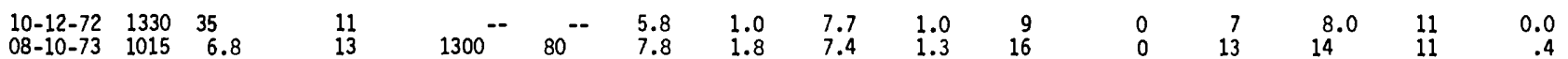
01109225 Rocky Run near Rehoboth, Mass. (Lat 414652 Long 0711505 )

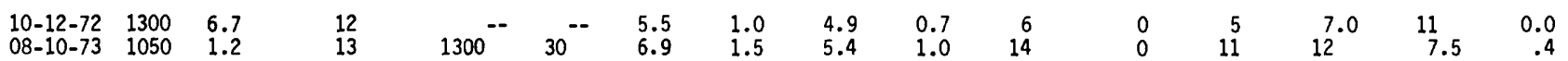

Runnins River basin

01109270 Runnins River at Seekonk, Mass. (Lat 414925 Long 0712000 )

$\begin{array}{llllllllllllllll}08-10-73 & 1140 & 1.2 & 13 & 1100 & 220 & 13 & 2.1 & 14 & 2.9 & 31 & 0 & 25 & 22 & 16 & 0.4\end{array}$


Table 6.--Chemical analyses at gaging stations and partial-record stations (continued)

\begin{tabular}{|c|c|c|c|c|c|c|c|c|c|c|c|c|c|c|c|}
\hline Date & $\begin{array}{l}\text { Dis- } \\
\text { solved } \\
\text { nitrate } \\
(\mathrm{N}) \\
(\mathrm{mg} / \mathrm{L})\end{array}$ & $\begin{array}{l}\text { Dis- } \\
\text { solved } \\
\text { nitrate } \\
(\mathrm{N}) \\
(\mathrm{mg} / \mathrm{L})\end{array}$ & $\begin{array}{l}\text { Dis- } \\
\text { solved } \\
\text { ammonia } \\
\text { nitro- } \\
\text { gen } \\
(\mathrm{N}) \\
(\mathrm{mg} / \mathrm{L})\end{array}$ & $\begin{array}{c}\text { Organic } \\
\text { nitro- } \\
\text { gen } \\
(\mathrm{N}) \\
(\mathrm{mg} / \mathrm{L})\end{array}$ & $\begin{array}{l}\text { Total } \\
\text { Kjel- } \\
\text { dahl } \\
\text { nitro- } \\
\text { gen } \\
(\mathrm{N}) \\
(\mathrm{mg} / \mathrm{L})\end{array}$ & $\begin{array}{l}\text { Dis- } \\
\text { solved } \\
\text { ortho- } \\
\text { phos- } \\
\text { phorus } \\
\text { (P) } \\
(\mathrm{mg} / \mathrm{L})\end{array}$ & $\begin{array}{l}\text { Total } \\
\text { phos- } \\
\text { phorus } \\
(\mathrm{P}) \\
(\mathrm{mg} / \mathrm{L})\end{array}$ & $\begin{array}{l}\text { Dis- } \\
\text { solved } \\
\text { solids } \\
\text { (sum of } \\
\text { constit- } \\
\text { uents } \\
\text { (mg/L) }\end{array}$ & $\begin{array}{l}\text { Hard- } \\
\text { ness } \\
(\mathrm{Ca}, \mathrm{Mg}) \\
(\mathrm{mg} / \mathrm{L})\end{array}$ & $\begin{array}{c}\text { Non- } \\
\text { car- } \\
\text { bonate } \\
\text { hard } \\
\text { ness } \\
(\mathrm{mg} / \mathrm{L})\end{array}$ & $\begin{array}{l}\text { Specific } \\
\text { conduct- } \\
\text { ance } \\
\text { (micro- } \\
\text { mhos) }\end{array}$ & $\begin{array}{c}\mathrm{pH} \\
\text { (units) }\end{array}$ & $\begin{array}{l}\text { Tem- } \\
\text { pera- } \\
\text { ture } \\
\left({ }^{\circ} \mathrm{C}\right)\end{array}$ & $\begin{array}{l}\text { Carbon } \\
\text { dioxide } \\
\left(\mathrm{Co}_{2}\right) \\
(\mathrm{mg} / \mathrm{L})\end{array}$ & $\begin{array}{l}\text { Color } \\
\text { (plat- } \\
\text { inum- } \\
\text { cobalt } \\
\text { units) }\end{array}$ \\
\hline
\end{tabular}

East Branch Westport River basin

01105937 Shingle Island River at $01 d$ Fall River Road (Lat 414050 Long 0710105.01 )

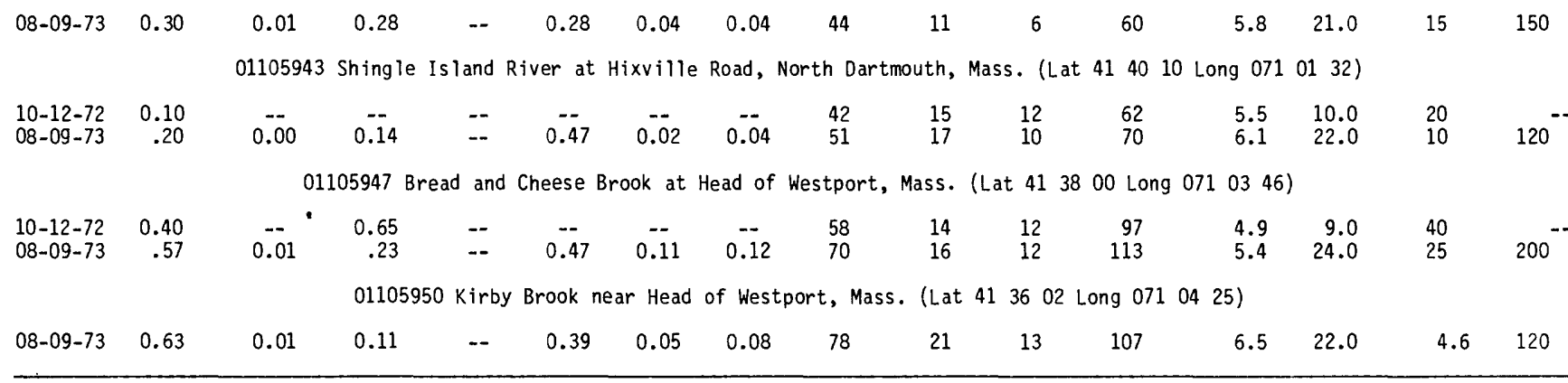

West Branch Westport River basin

01106000 Adamsville Brook at Adamsville, R.I. (Lat 413330 Long 0710747 )

$\begin{array}{rrrrrrrrrrrrrrrrrrrr}10-12-72 & .09 & -- & .40 & -- & -- & -- & -- & 46 & 15 & 10 & 68 & 6.1 & 11.0 & 7.6 & -.0 \\ 08-09-73 & .30 & .01 & .16 & -- & 0.39 & 0.07 & 0.08 & 56 & 13 & 8 & 77 & 6.2 & -- & 7.1 & 200\end{array}$ 01106005 Angeline Brook near Westport Point, Mass. (Lat 413305 Long 07106 20)

$\begin{array}{ccccccccccccccccc}10-12-72 & 1.1 & -- & -- & -- & -- & -- & -- & 58 & 24 & 20 & 86 & 6.0 & 11.0 & 6.4 & -- \\ 08-09-73 & 1.9 & 0.00 & 0.07 & -- & 0.55 & 0.04 & 0.05 & 83 & 32 & 25 & 120 & 6.4 & 21.0 & 5.1 & 100\end{array}$ Lee River basin

01109130 Lewin Brook at Swansea, Mass. (Lat 414455 Long 0711145.01 )

\begin{tabular}{llllllllllllllllll}
$08-10-73$ & 0.14 & 0.00 & 0.11 & -- & 0.48 & 0.02 & 0.03 & 55 & 26 & 7 & 89 & 6.9 & 22.0 & 4.6 & 80 \\
\hline
\end{tabular}

Cole River basin

01109135 Cole River near Swansea, Mass. (Lat 414630 Long 0711200 )

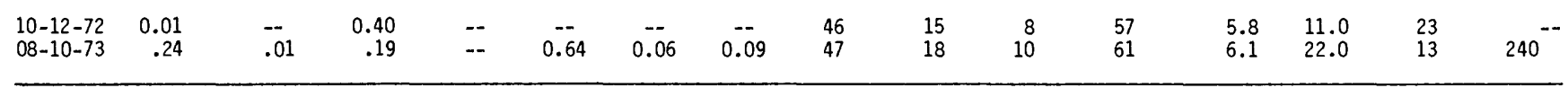

Palmer River basin

01109195 East Branch Palmer River at Rehoboth, Mass. (Lat 415030 Long 0711435.01 )

$\begin{array}{llllllllllllllllll}08-10-73 & 0.18 & 0.00 & 0.11 & - & 0.50 & 0.03 & 0.03 & 57 & 18 & 10 & 76 & 6.7 & 22.0 & 3.2 & 55\end{array}$

01109200 West Branch Palmer River near Rehoboth, Mass. (Lat 415246 Long 07115 18)

$\begin{array}{rrrrrrrrrrrrrrrrrr}10-12-72 & 0.10 & -. & 0.90 & -- & -- & -- & -- & 46 & 16 & 9 & 61 & 6.6 & 10.0 & 3.6 & --0 \\ 08-10-73 & .09 & 0.00 & .11 & -- & 0.51 & 0.03 & 0.04 & 57 & 24 & 11 & 76 & 6.5 & -- & 7.6 & 170\end{array}$ 01109215 Palmer River near Rehoboth, Mass. (Lat 414950 Long 0711655 )

$\begin{array}{ccccccccccccccccc}10-12-72 & 0.10 & -- & -- & -- & -- & -- & - & 50 & 19 & 11 & 68 & 7 & 9.5 & 1.4 & -- \\ 08-10-73 & -- & 0.00 & 0.08 & -- & 0.24 & 0.02 & 0.03 & 68 & 27 & 14 & 97 & 6.7 & 21.0 & 5.1 & 80\end{array}$ 01109225 Rocky Run near Rehoboth, Mass. (Lat 414652 Long 0711505 )

$\begin{array}{rrrrrrrrrrrrrrrrrr}10-12-72 & 0.09 & -- & 0.59 & -- & -- & -- & -- & 46 & 18 & 13 & 57 & 5.8 & 11.0 & 15 & -0 \\ 08-10-73 & .28 & 0.01 & .14 & -- & 0.62 & 0.01 & 0.01 & 56 & 23 & 12 & 79 & 6.4 & 24.0 & 8.9 & 160\end{array}$

Runnins River basin

01109270 Runnins River at Seekonk, Mass. (Lat 414925 Long 0712000 )

$\begin{array}{llllllllllllllllllll}08-10-73 & 1.1 & 0.01 & 0.10 & - & 0.58 & 0.47 & 0.58 & 105 & 41 & 16 & 164 & 7.2 & 21.0 & & & & \end{array}$


Information from annual reports and records of municipal water systems, water districts, Bristol County Water Company, and the Massachusetts Department of Public Health. Local well number of each station, in parentheses, can be used to locate the well (pl. I) and to refer to well description (table 1), log of materials (table 2), and partial or complete chemical analyses (tables 3 and 6).

ATTLEBORO. Municipal system with both surface-water and ground-water sources in Tenmile basin (Williams and Willey, 1967, table 5) supplies part of city within area. No ground-water exploration or pumping facilities in area.

BRISTOL COUNTY WATER COMPANY. A subsidiary of American Waterworks Co., serves (in 1965) 16,260 people in Barrington, R.I., 15,500 people in Bristol, R. I., and 9,000 people in Warren, R.l. System demand in 1965 was about $3.80 \mathrm{Mgal} / \mathrm{d}$, and yield of reservoirs was 4.50 $\mathrm{Mgal} / \mathrm{d}$. Surface supply supplemented by wells in Rhode Island.

Surface-water supply: Storage of in Upper Warren Reservoir and Shad Factory Pond on Palmer River can be released via pipeline to pumping station at lower end of (Warren Reservoir) Kickamuit Reservoir in Rhode Island which also receives water from Warren Reservoir via Kickamuit River. The lowermost reservoir in the system, in Warren R.I. (Kickamuit Reservoir), is subject to saltwater flooding during hurricane tides.

Source of data: Rhode Island Statewide Comprehensive Transportation and Land Use Planning Program, 1969, Plan for the development and use of public water supplies: Report 10, figure 2 and p. 23, The State House, Providence, R.I.

DARTMOUTH. Municipal system serving about 6,000 users. Until 1961, when town wells were placed in service, supply furnished by New Bedford through a connection at Allen Avenue and Rockdale Street. After 1961, New Bedford supply used to meet summer peak loads, but increasing demand has placed greater demands on New Bedford supply since 1970; Dartmouth may take as much as $5 \mathrm{Mgal} / \mathrm{d}$. Improvements are currently under construction to increase the quantity of water available from the New Bedford system. In 1972, wells produced $357 \mathrm{Mgal}$; New Bedford supplied 137 Mgal.

Well A off Chase Road (outside basin), a gravel-packed well having a yield of about $0.5 \mathrm{Mgal} / \mathrm{d}$.

Wells B and C off Chase Road (outside basin), 24-inch pumping gravel-packed well connected to a 10-inch gravel-packed siphon well about 200 feet to the east. Estimated yield, $370 \mathrm{gal} / \mathrm{min}$ or $0.53 \mathrm{Mgal} / \mathrm{d}$.

Route 6 well (DCW-93). (1963), a 16-inch gravel-packed well yielding $340 \mathrm{gal} / \mathrm{min}$ or nearly $0.5 \mathrm{Mgal} / \mathrm{d}$.

New Bedford water system (outside basin)

Additional sources: Violetta well site (outside basin) tested at about $250 \mathrm{gal} / \mathrm{min}$ in T961, but believed incapable of continuous production; engineers suggested installation of peak-load well capable of $500 \mathrm{gal} / \mathrm{min}$ for short periods.

DIGHTON WATER DISTRICT. Serves all of Dighton except for northern part supplied by North Dighton Water District from its interconnection with the Taunton water system. In 1972, about half of the pumpage of the Dighton Water District was furnished by the Taunton water system, and the balance was furnished by a well field outside the basin near the Taunton River, and from the Cedar Street station. 
Table 7.-Description of public water-supply sources (continued)

DIGHTON WATER DISTRICT-continued

Cedar Street station, a 48 × 24-inch gravel-packed well (DTW-318) with pump capacity of $300 \mathrm{gal} / \mathrm{min}$.

Well field off Route 138 (Taunton River basin), 10 2-1/2-inch wells yielding about $100 \mathrm{gal} / \mathrm{min}$.

Additional sources: Possible site 700 feet south of Cedar Street well, where group pump test yielded $100 \mathrm{gal} / \mathrm{min}$.

FALL RIVER. Municipal system managed by Watuppa Water Board. Serves about 100,000 people in Fall River and certain contiguous areas.

Reservoir system:

North Watuppa Pond, supplies drinking water, supplemented by water from Copicut Reservoir and Noquochoke Lake.

South Watuppa Pond, industrial supply, occasionally supplemented by water from North Watuppa Pond, and augmented by water from Noquochoke Lake in Dartmouth. Augmented by overflow from Stafford Pond and Sawdy Pond.

Noquochoke Lake, connected via pipeline beneath Route 6 to Watuppa Ponds to supplement industrial supply and occasionally the drinking water supply.

Copicut Reservoir, in service 1972, to provide additional supply to North Watuppa Pond drinking water supply.

Additional sources: City retains rights to water of Long Pond, one of the Lakeville Ponds, granted under Act of 1924 dividing water among Taunton, Fall River, and New Bedford.

Shingle Island Reservoir (proposed) in Dartmouth.

FREETOWN. No public water system; municipal buildings served by individual wells. About 800 persons in East Freetown are supplied by the New Bedford water system.

REHOBOTH. No public-supply system; municipally owned buildings supplied by individual wells.

SEEKONK WATER DISTRICT. Organized 1945, supplies Town of Seekonk.

Brown Avenue well field (1945) consists of 43 2-1/2-inch, driven, open-end wells (SHW-178); pump capacity $450 \mathrm{gal} / \mathrm{min}$, yield $0.36 \mathrm{Mgal} / \mathrm{d}$.

Newman Avenue station: Gravel-packed well $48 \times 24$ inches (1953) yielding I Mgal/d (SHW 265); gravel-packed well $48 \times 24$ inches (1958) yielding I Mgal/d (SHW 266); gravel-packed well $48 \times 24$ inches (1970) yielding I Mgal/d (SHW 3/3).

SOMERSET. Municipal system established about 1913, with Pierce Lane well (SPW 148); well field constructed off Brook Street in Dighton about 1926 (DTW 218), and two gravel-packed wells constructed about 1949-52 (DTW 216 and DTW 217); and a $5 \mathrm{Mgal} / \mathrm{d}$ reservoir, diversion works from Segraganset River, and treatment plant, in service 1967--all in Taunton River basin. No water-supply sources in coastal drainage basins.

WESTPORT. No municipal public-supply system; municipally owned buildings supplied by private wells. Individual community supplies at Westport Point and Acoaxet.

Additional sources: Exploration by test wells in 1964-65 located one site capable of yielding $0.45 \mathrm{Mgal} / \mathrm{d}$. 
An asterisk indicates that the report is out of print but may be consulted at the above offices and at many public and educational institution libraries. These reports are available to be viewed at the following U.S. Geological Survey offices:

\author{
U.S. Geological Survey \\ Water Resources Division \\ I50 Causeway Street, Suite 1001 \\ Boston, MA 02114 \\ (Massachusetts reports only)
}

\author{
U.S. Geological Survey \\ Clinton Street, NH Rt. 13, Bow, NH 0330। \\ Mailing address: RFD 2, Box 352A \\ Concord, $\mathrm{NH} 03301$ \\ (New Hampshire reports only)
}

\author{
1J.S. Geological Survey \\ Water Resources Division \\ 26 Ganneston Drive \\ Augusta, ME 04330 \\ (Maine reports only)
}

MASSACHUSETTS

* Wilmington-Reading Area, by John A. Baker and Edward A. Sammel, 1961, 50 p., 2 figs. Covers an area of about 43 square miles in the upper part of the Ipswich River basin in northeastern Massachusetts.

*2 Lower Ipswich River basin, by Edward A. Sammel and John A. Baker, 1962, 47 p., 2 figs. Covers an area of about 110 square miles in northeastern Massachusetts.

*3 Lowell Area, by John A. Baker and Richard G. Petersen, 1962, 28 p., 2 figs. Covers an area of about II5 square miles and includes most of the metropolitan area of the City of Lowell.

*4 Parker and Rowley River basins, by Edward A. Sammel, 1962, 33 p., 2 figs. The rivers drain an area of about 77 square miles in northeastern Massachusetts.

*5 Brockton-Pembroke Area, by Richard G. Petersen, 1962, 46 p., 2 figs. Covers an area of about $1 / 2$ square miles in the northern part of Plymouth County

*6 Western Massachusetts, by Richard G. Petersen and Anthony Maevsky, 1962, 21 p., 1 fig. Covers an area of about 2,865 square miles and includes all of Berkshire, Franklin, Hampshire, and Hampden Counties.

*7 Southeastern Massachusetts, by Anthony Maevsky and Janet A. Drake, 1963, 55 p., 2 figs. Covers an area of about 1,930 square miles and includes all of Barnstable, Bristol, Dukes, Nantucket, and Plymouth Counties (exclusive of the Brockton-Pembroke Area).

*8 Assabet River basin, by Samuel J. Pollock and William B. Fleck, 1964, 45 p., I pl. Covers an area of approximately 177 square miles and includes parts of Middlesex and Worcester Counties.

*9 Housatonic River basin, by Ralph F. Norvitch and Mary E. S. Lamb, 1966, 50 p., I pl. Covers an area of about 530 square miles in the upper part of the basin, which is north of the Connecticut-Massachusetts State line.

* 10 Northern part, Ten Mile and Taunton River basins, by John R. Williams and Richard E. Willey, 1967,56 p., I pl., 1 fig. Covers an area of about 195 square miles within Bristol, Norfolk, and Plymouth Counties.

* II Millers River basin, by Donald R. Wiesnet and William B. Fleck, 1967, 29 p., I pl., I fig. Covers an area of about 392 square miles within Franklin and Worcester Counties, Massachusetts, and Hillsborough and Cheshire Counties, New Hampshire.

* 12 Taunton River basin, by John R. Williams and Richard E. Willey, 1970, 102 p., I pl., I fig. Covers an area of about 528 square miles in Bristol, Norfolk, and Plymouth Counties.

13 Deerfield River basin, by Bruce P. Hansen, Frederick B. Gay, and L. G. Toler, 1973, 59 p., I fig., I pl. Covers an area of 348 square miles in northwestern Massachusetts

14 Neponset and Weymouth River basins, by R. A. Brackley, William B. Fleck, and Richard E. Willey, 1973, 51 p., I fig., I pl. Covers an area of 183 square miles in eastern Massachusetts south of Boston.

15 Hoosic River basin, by Bruce P. Hansen, Frederick B. Gay, and L. G. Toler, 1974, 33 p., I pl., I fig. Covers an area of 164 square miles in northwestern Massachusetts

16 Weir River, Hingham, to Jones River, Kingston, by John R. Williams, Richard E. Willey, and Gary D. Tasker, 1975, 63 p., I pl., I fig. Principal basins covered are those of Weir River, James Brook, Bound Brook, North River, South River, and Jones River.

17 Ground-water levels in Massachusetts, 1936-74, by Anthony Moevsky, 1976, 107 p., 2 figs. Documents both short-term and long-term ground-water-level trends in typical hydrologic situations and different geographic areas of the Commonwealth.

18 Plymouth to Weweantic River, Wareham, by John R. Williams, Gary D. Tasker, and Richard E. Willey, 1977,31 p., I pl., I fig. Principal basins are Town Brook, Eel River, and Beaverdam Brook, all draining to Cape Cor Bay; Herring Brook draining to the Cape Cod Canal; and Red Brook, Agawam River, Wankinco River, and Weweantic River, all draining to Buzzards Bay.

19 Charles River basin, by Eugene H. Walker, William W. Caswell, and S. William Wandle, Jr., 1977, 53 p., I pl., I fig. Covers an area of abqut 300 square miles of eastern and southeastern Massachusetts within the counties of Middlesex, Norfolk, Suffolk, and Worcester.

20 Northwest Shore of Buzzards Bay, by John R. Williams, Richard E. Willey, and Gary D. Tasker, 1980, 30 p., I pl. Principal drainage basins are Sipican River, Aucoot Brook, Mattapo isett River, Acushnet River, and Paskamansett River.

21 Coastal drainage basins of northeastern Massachusetts, from Castle Neck River, Ipswich, to Mystic River, Boston, by David $F$. Delaney and Frederick B. Gay, 1980, 40 p., I pl. Principal streams are the Annisquam, Castle Neck, Danvers, Essex, Mystic, and Saugus Rivers, which flow into Ipswich and Massachusetts Bays.

22 Shawsheen River basin by David F. Delaney and Frederick B. Gay, 1981, 22 p., I pl. Principal tributaries are Content, Elm, Heath, Hussey, Kiln, Rogers, Spring, Strong Water, Vine and Webb Brooks.

23 Lake Cochituate drainage basin, Framingham-Natick, Massachusetts, by Frederick B. Gay, 1981, 61 p., 1 pl. Covers 17.7 square miles above the outlet of Lake Cochituate which includes Beaverdam, Course, Pegan, and Snake Brooks, and Fisk Pond.

24 Lower Merrimack River basin, from Concord River, Lowell, to Plum Island, Newburyport, by David F. Delaney and Frederick B. Gay, 198I, $34 \mathrm{p.}$, I pl. Principal tributaries are Bare Meadow, Bartlett, Cobbler Creek, Fish, Richardson, and Trull Brooks, Artichoke, East Meadow, Indian, Little, Powwow, and Spicket Rivers, and Johnson Creek. The Blackwater River basin is included in the report.

NEW HAMPSHIRE

*1 Southeastern Area, by Edward Bradley and Richard G. Petersen, 1962, 53 p., 5 figs. Covers an area of about 390 square miles in parts of Rockingham and Strafford Counties.

2 Lower Merrimack River valley, by James M. Weigle and Richard Kranes, 1966, 44 p., I pl. Covers an area of about 396 square miles in central-southern New Hampshire.

3 Ashuelot River basin, by Harold A. Whitcomb, 1973, 25 p., 1 pl. Covers an area of about 420 square miles in southwestern New Hampshir

MAINE

Southwestern Area, by Glenn C. Prescott, Jr., and Janet A. Drake, 1962, 35 p., 2 figs. Covers an area of about 800 square miles in York County.

2 Lower Penobscot Basin, by Glenn C. Prescott, Jr., 1964, 40 p., 3 figs. Covers an area of about 825 square miles in Penobscot, Hancock, and Waldo Counties.

3 Lower Androscoggin River basin, by Glenn C. Prescott, Jr., 1967, 63 p., 2 figs. Covers most of Androscoggin County, a large part of Oxford County, and portions of Cumberland, Kennebec, and Sagadahoc Counties.

4 Lower Kennebec River basin, by Glenn C. Prescott, Jr., 1968, 38 p., 2 figs. Covers most of Kennebec County, more than half of Sagadahoc County, and portions of Androscoggin, Franklin, Lincoln, and Somerset Counties.

5 Lower Aroostook River basin, by Glenn C. Prescott, Jr., 1970, 30 p., 2 figs. Covers an area of about 536 square miles in northeastern Aroostook County.

6 Lower St. John River valley, by Glenn C. Prescott, Jr., 1971; 22 p., 2 figs. Covers an area of about 204 square miles at the northem border of Maine.

7 Meduxnekeag River-Prestile Stream basins, by Glenn C. Prescott, Jr., 197I, 17 p., 2 figs. Covers an area of about 312 square miles in Aroostook County.

8 Southern Washington County, by Glenn C. Prescott, Jr., 1973, 40 p., 2 figs. Covers an area of about 720 square miles in Washington County and about 10 square miles in Hancock County.

9 Windham-Freeport-Portland Area, by Glenn C. Prescott, Jr., 1976, 48 p., 2 figs. Covers an area of about 450 square miles in Cumberland County. 